\title{
A General Picture of Cucurbit[8]uril Host-Guest Binding: Recalibrating Bonded Interactions
}

\author{
Zhaoxi Sun ${ }^{1 *}$, Qiaole $\mathrm{He}^{2}$, Zhihao Gong ${ }^{1}$, Payam Kalhor ${ }^{1}$, Zhe Huai ${ }^{3 *}$, Zhirong Liu ${ }^{1}$

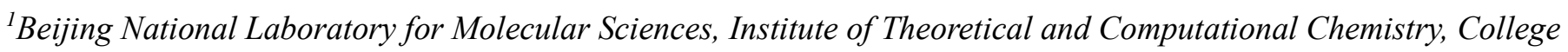 \\ of Chemistry and Molecular Engineering, Peking University, Beijing 100871, China \\ ${ }^{2}$ AI Department of Enzymaster (Ningbo) Bio-Engineering Co., Ltd., North Century Avenue 333, 315100 Ningbo, \\ China \\ ${ }^{3}$ XtalPi - AI Research Center (XARC), 9F, Tower A, Dongsheng Building, No.8, Zhongguancun East Road, Haidian \\ District, Beijing 100083, P.R. China
}

*To whom correspondence should be addressed: z.sun@pku.edu.cn, zhe.huai@xtalpi.com

\begin{abstract}
Atomic-level understanding of the dynamical feature of host-guest interactions remains a central challenge in supramolecular chemistry. The remarkable guest binding behavior of the Cucurbiturils family of supramolecular containers makes them promising drug carriers. Among Cucurbit $[n]$ urils, Cucurbit[8]uril (CB8) has intermediate portal size and cavity volume. It can exploit almost all host-guest recognition motifs formed by this host family. In our previous work, an extensive computational investigation of the binding of 7 commonly abused and structurally diverse drugs to the CB8 host was performed and a general dynamical binding picture of CB8-guest interactions was obtained. Further, two widely used fixed-charge models for drug-like molecules were investigated and compared in great detail, aiming at providing guidelines in choosing an appropriate charge scheme in host-guest modelling. Iterative refitting of atomic charges leads to improved binding thermodynamics and the best root-mean-squared deviation from the experimental reference is $2.6 \mathrm{kcal} / \mathrm{mol}$. In this work, we focus on a thorough evaluation of the remaining parts of classical force fields, i.e., the bonded interactions. The widely used general Amber force fields are assessed and refitted to improve the intra-molecular conformational preference and thus the description of inter-molecular host-guest interactions. The interaction pattern and binding thermodynamics show significant dependence on
\end{abstract}


the modelling parameters. The refitted system-specific parameter set improves the consistency of the modelling results and the experimental reference significantly. Finally, combining the previous chargescheme comparison and the current force-field refitting, we provide general guidelines for the theoretical modelling of host-guest binding.

Keywords: Cucurbit[8]uril, Host-guest Interaction, Binding Mode, Force Fields, Abused Drugs 


\section{Introduction}

Pharmaceutical research acts as a key component in biological and medical science. Great efforts are required to grasp some basic insights of druggable species from the universe of chemical space. By definition, drugs are small molecules that incur physiological or psychological changes in specific target organism(s). Despite their small sizes, they are able to regulate the functionality of huge biomacromolecular receptors upon binding to specific area(s) of the targeted molecule or its biologically relevant counterparts. ${ }^{1-5}$ The consumption of drugs should follow designed prescriptions to avoid overdosage, misuse and abuse. Otherwise, injuries in various organisms would be triggered. ${ }^{6-14}$

The family of the pumpkin-like supramolecular container Cucurbit $[n]$ uril $(\mathrm{CB}[n])$ is formed by $n$ glycoluril monomers linked by $2 n$ methylene bridges. Their hydrophilic carbonyl portals on glycoluril units stabilize cationic species and act as hydrogen bond acceptors, while the central partly closed hydrophobic cavity is able to coordinate aliphatic and aromatic components. As a result, this series of heterocycles are able to form stable host-guest complexes with a wide range of neutral and cationic species with diverse structural features, which makes them promising coordinators and carriers of functional species including drugs. ${ }^{15-23}$ Among the $\mathrm{CB}[n]$ family, Cucurbit[8]uril (CB8 or $\mathrm{CB}[8]$, see Fig. 1a) has intermediate portal size and cavity volume, which enables it to exploit almost all host-guest recognition motifs formed by this host family. Thus, structural and thermodynamic understandings of CB8-guest interactions would be useful to elucidate the functionality of the whole family.

In molecular simulations of condensed matter systems especially biological systems, the high dimensional space needs to be sampled extensively in order to reach the ergodic assumption. ${ }^{24-27}$ The accuracy of time integration of Hamilton equations limits the time step to several $\mathrm{fs},{ }^{28-32}$ while the phenomena of interest normally happen at $\mu \mathrm{s}, \mathrm{ms}$ and even longer time scales. ${ }^{33-39}$ However, molecular dynamics (MD) simulations of biological molecules with modern computational resources accessible to ordinary researchers can only achieve $\mu$ s, which is still far from the biologically relevant time scales. The spirit of proper sampling in the conformational space, often named as importance sampling, significantly extends the accessible time horizon. ${ }^{40-45}$ There are many ways to improve the sampling efficiency. For instance, biasing potentials are added to the system under investigation to restrain the sampling to specific regions of phase space or enable a more flexible walk on the potential energy surface. ${ }^{46-51}$ The important slow degree of freedom that the biasing potential is added on is referred to as reaction coordinate or collective variable (CV), which is selected intuitively or according to some statistical analyses. ${ }^{52-59}$ As the biasing potential modifies the energy function of the original system, a post-processing procedure is required to recover the statistics in the unbiased 
ensemble, which is often performed with estimators constructed from free energy perturbation. An alternative route to deal with the time-scale problem is the alchemical method, which constructs an artificial pathway connecting states of interest. ${ }^{60-64}$ Although the method could be successful when the conformational ensemble relevant to the alchemical transformation is simple, only limited thermodynamic information could be extracted. In cases that the conformational ensemble is rather complex with many hidden barriers, still enhanced sampling in the configurational space is required. Reliable statistics could be obtained with converged sampling results, but the accuracy of the simulation results depends on another factor in molecular modelling, i.e., the Hamiltonian or model used to describe the simulated system. Although the quantum mechanics $(\mathrm{QM})$ treatment is accurate and transferrable, ${ }^{65-70}$ all-atom molecular mechanics (MM) force fields are widely employed for biological systems due to efficiency considerations and the size, complexity, and intrinsic time scale of the investigated problem. ${ }^{71-76}$

Due to the symmetric feature and the simplicity of the CB8 structure, most modelling works of similar host-guest systems employ a priori that only a single host-guest interaction mode exists. ${ }^{21,77-82}$ In this way, the computational modelling of host-guest interactions could be simplified to a single-state calculation, where single-point calculations with normal mode approximation or restrained sampling in the neighborhood of the initial bound conformation could be employed without the introduction of significant systematic bias. However, in our previous extensive modelling of similar host-guest systems, complex conformational ensemble is observed. ${ }^{83,84}$ The conformational fluctuations of the individual components involved in hostguest interaction, the orientation of specific functional groups, and the diverse structural features of guests could lead to the existence of multiple unexpected binding modes, the free energy barriers between which are well above the thermal energy at physiological condition. Therefore, such a single-mode assumption is generally invalid, even for structurally simple host-guest systems.

In our previous work, the binding of 7 commonly abused drugs to the CB8 host were investigated extensively. ${ }^{83}$ The simulation does not rely on such single-state approximation but explores the configurational space extensively. The structural features of the considered drugs (G1 Metamfetamine, G2 Fentanyl, G3 Morphine, G4 Hydromorphone, G5 Ketamine, G6 Phencyclidine/PhenylCyclohexylPiperidine, and G7 Cocaine, see Fig. 1a) are quite diverse, which makes it possible to conclude a general picture of the CB8-guest binding. The computational perspective generally suggests that the host tends to become squashed to hold the guest tightly, and such host-guest coordination is dynamical with many highly populated interaction modes. Although the previous work provides valuable insights into CB8-guest binding and host-guest modelling, further developments are still in sight. 
All-atom force fields describe inter-molecular interactions with electrostatics and vdW terms, the former of which contributes the most significant part of the non-bonded interactions. Changes in atomic charges could perturb the local interaction network and thus lead to significantly different thermodynamic profiles. ${ }^{85-89}$ The polarization effect due to changes in the local environment could also play an important role in molecular recognition..$^{90-92}$ Two widely used charge schemes are assessed extensively for CB8-drug complexes in our previous work, and the restrained fitting method targeting the molecular electrostatic potential (ESP) is observed to provide better performance for binding affinity. To further improve the accuracy of electrostatics, polarizable models and even QM calculations are needed. Aside from the non-bonded terms that are directly involved in the calculation of inter-molecular interaction energies, the other terms describing the intramolecular interactions are also crucial to properly describe the molecular ensemble. Different from the nonbonded interactions, these bonded terms describe the stiffer or harder degrees of freedom inside each molecule. Among the stiff degrees of freedom, the dihedral term is relatively soft, and plays a key role in the conformational flexibility of biological macromolecules and small drug-like molecules. A balanced description of intra- and inter-molecular interactions (i.e., bonded and non-bonded interactions) is required to properly describe the inter-molecular interaction pattern. Therefore, in this work, we focus on the assessment and recalibration of the intra-molecular interaction terms. The general Amber force field (GAFF) ${ }^{93}$ and its derivatives are widely applied to describe the bonded terms in the modelling of drug-like molecules and thus also host-guest systems. ${ }^{94-97}$ Therefore, in the current work, we assess the first and second generations of GAFF (GAFF 1.81 and 2.11, to be specified) extensively for CB8-drug complexes. A worth noting deficiency of these force fields is that they are pre-fitted and aim at providing transferable descriptions for almost all drug-like molecules and thus could be of relatively low accuracy for specific systems. Thus, we also consider refitting the bonded terms with the generalized force-matching (FM) $\operatorname{method}^{98,99}$ for the host and guest molecules under investigation. The resulting refitted force field is expected to provide a description closer to the target Hamiltonian (i.e., the QM Hamiltonian used to re-generate the parameters of the bonded terms) and thus more accurate than the highly transferable GAFF derivatives. Combining the central results in the current bonded-term recalibration and our previous charge-scheme assessment, some useful guidelines for the modeling of CB8 host-guest systems could be summarized. More generally, the results are expected to be useful in the modelling of all host-guest complexes.

\section{Methodology and Computational Details}

\subsection{System preparation.}


The 3D structures of host CB8 and its 7 commonly abused drugs are obtained from our previous work. ${ }^{83}$ The 3D structure of the host CB8 and the 2D chemical structures of the guest molecules are shown in Fig. 1a. The experimental binding affinities ${ }^{100}$ of these host-guest systems are summarized in Table 1. In model construction, we first need to determine the protonation states of each molecule. The experimental and ChemAxon predicted pKa of each molecule is summarized in Table 1, with which the protonation state of each molecule is determined. The host CB8 is net-neutral, and all guest molecules are protonated except G5 Ketamine. Due to the closeness of the experimental and ChemAxon predicted $\mathrm{pKa}$ of this molecule and the $\mathrm{pH}$ condition ( $\mathrm{pH} 7.4$ ) that the experimental binding affinities are measured, there should be an acid-base equilibrium in the CB8-G5 binding. Thus, both the protonated and deprotonated forms of G5 are modelled in our computational investigation. The net charges of the host and the guest molecules are summarized in Table 1.

According to our previous work on CB8-drug systems, the reproduction of the molecular ESP is better with the restrained electrostatic potential (RESP) ${ }^{101}$ charge scheme than the corrected semi-empirical AM1$\mathrm{BCC}^{102}$ charges. Thus, the inter-molecular interactions are more accurate with RESP charges. Further, the deviations of the predicted binding thermodynamics from the experimental values are smaller with RESP charges than AM1-BCC and the ranking coefficients are also better for the RESP charge scheme. Therefore, we employ the RESP charge scheme to represent electrostatics in the current work. The performance of the RESP charge scheme shows obvious dependence on the guest conformation used in charge generation, as shown in our previous calculations. ${ }^{83}$ On this aspect, the proposed iterative refitting procedure for the generation and calibration of atomic charges would be helpful. After one round of such iterative refitting, the calculated binding affinities obtained with the new RESP charge set are much closer to the experimental reference. ${ }^{83}$ Thus, we choose this 'best' set of atomic charges to describe the electrostatics in the current work. The other force-field parameters including the vdW interactions and the bonded terms (e.g., bond stretching and dihedral) are obtained from GAFF (GAFF version 1.81) or the second generation of GAFF (GAFF version 2.11, GAFF2). ${ }^{93}$ As the GAFF derivatives are parameterized to achieve high transferability, their accuracies for specific systems could be relatively low compared with force fields parameterized for specific systems. To further improve the quality of the model, we also parameterize system-specific force fields for the bonded terms to achieve a higher level of accuracy. The generalized FM scheme ${ }^{98,99}$ is used in this bonded-term refitting, the details of which would be discussed in the results part. The starting structure of each host-guest complex is obtained by simply superposing the center of masses (COM) of the host and that of the guest. Solvation is performed with TIP3P $\mathrm{P}^{103,104}$ water and the minimum distance between the 
solute surface and the box edge is set to $25 \AA$, which is sufficiently large to define a decoupled state. The truncated octahedron cell is replicated in whole space with periodic boundary conditions. Non-polarizable monovalent spherical counter ions ${ }^{105,106}$ of $\mathrm{Na}^{+}$or $\mathrm{Cl}^{-}$parameterized for TIP3P water are added for neutralization.

\subsection{Free Energy Simulations.}

As the macrocyclic host CB8 is structurally symmetric and much simpler than biomacromolecular receptors (e.g., protein), it is often presumptive that there is only one stable binding mode in each CB8-guest

complex. ${ }^{21,77-81}$ However, the validity of such a priori for practical systems remains unknown unless extensive exploration of the configurational space is performed. As observed in our previous extensive modelling of host-guest systems, ${ }^{83,84,97,107}$ the CB8-guest complex has a quite complex conformational ensemble, where multiple binding modes with similar thermodynamic stabilities would contribute significantly. Despite the symmetric structural feature of the macrocyclic host, the conformational fluctuations of the host and the guest, the orientation of specific functional groups, and the diverse structural features of the guest molecules would make converged sampling very difficult. The high dimensionality of the host-guest system and the high free energy barrier to overcome when exploring the decoupled state call for the use of enhanced sampling techniques. To improve the sampling efficiency, we employ well-tempered metadynamics. ${ }^{108-111}$ Then, another question is how to deposit or distribute the biasing potential in order to explore the space of host-guest binding modes extensively. To avoid any priori of the relative host-guest position and thus the detailed interaction feature of binding modes, the biasing $\mathrm{CVs}$ are chosen as the spherical coordinates $(\rho, \theta, \varphi)$ defined by the COMs of the host and the guest. ${ }^{83}$ When calculating the absolute binding affinity, an entropic correction term is also needed to recover the standard-state condition. For more detailed discussions about the sampling protocol, please refer to our previous works on similar host-guest systems. ${ }^{83,84,97}$ An illustration of the 3D spherical CV is presented in Fig. $1 \mathrm{~b}$.

To provide a detailed analysis of the host-guest coordination pattern, we calculate the contact number

between groups of atoms, which is defined as $C=\sum_{i \in \text { group }_{\mathrm{A}}} \sum_{j \in \text { group }_{\mathrm{B}}} \frac{1-\left(\frac{r_{i j}}{r_{0}}\right)^{6}}{1-\left(\frac{r_{i j}}{r_{0}}\right)^{12}}$. Here, group A and group B are two groups of atoms involved in inter-molecular coordination. The distance constant in the switching function $r_{0}$ is set to $6 \AA$, which is consistent with our previous CB8 host-guest work. ${ }^{83}$ The variable $r_{i j}$ represents 
the distance between the $i$ th atom in group A and the $j$ th atom in group B. To monitor the closeness of the host and the guest during the exploration of the configurational space, we calculate the total contact number between the host and the guest $C_{\text {host-guest }}=\sum_{i \in \text { host }} \sum_{j \in \text { guest }} \frac{1-\left(\frac{r_{i j}}{r_{0}}\right)^{6}}{1-\left(\frac{r_{i j}}{r_{0}}\right)^{12}}$. More detailed information of host-guest coordination (e.g., atoms involved in the inter-molecular coordination) could be extracted from its by-hostatom decomposition $C_{i}=\sum_{j \in \text { guest }} \frac{1-\left(\frac{r_{i j}}{r_{0}}\right)^{6}}{1-\left(\frac{r_{i j}}{r_{0}}\right)^{12}}$, i.e., the atom-guest contact for the $i$ th atom of the host. Due to the complexity of the host-host interactions, the total host-guest contact is observed to be insufficient to differentiate different inter-molecular interaction patterns properly. ${ }^{83}$ Therefore, according to the chemical intuition that the large cavity of the CB8 ring needs to be filled with some parts of the guest and the fact that there is at least one 6-membered aromatic ring in each guest, we consider the contact number between the host and the aromatic ring $C_{\text {host-phenyl }}=\sum_{i \in \text { host }} \sum_{j \in \text { phenyl }} \frac{1-\left(\frac{r_{i j}}{r_{0}}\right)^{6}}{1-\left(\frac{r_{i j}}{r_{0}}\right)^{12}}$ to analyze detailed features of the binding modes. As there are two phenyl rings in G2, the selected phenyl ring is directly connected to the amide bond. Note that in free energy analysis, only heavy atoms are included in the calculation of contact numbers.

As has been discussed in system preparation, the starting configuration of each host-guest complex is obtained by simply superposing the COMs of the host and the guest. Although such a bound conformation could be energetically unfavorable and the system could be far from equilibrium, we perform 5000 steps minimization, 400 ps NVT equilibration, and $2 \mathrm{~ns}$ NPT equilibration to let various degrees of freedom relax and find their favorable regions. After that, we initiate $1000 \mathrm{~ns}$ spherical-coordinates-biased metadynamics simulations. The parameters for the metadynamics setting remain the same as our previous work. ${ }^{83,84,97}$ Namely, we use a $0.24 \mathrm{kcal} / \mathrm{mol}$ initial height of the repulsive Gaussian, the deposition interval of $0.5 \mathrm{ps}$, the bias factor of 20, and Gaussian widths of $0.1 \mathrm{~nm}, \frac{\pi}{16}$, and $\frac{\pi}{8}$ for the three polar coordinates, respectively. The simulation is performed with GROMACS $2019.6^{38}$ patched with PLUMED 2.7.0 $0^{112}$. The velocity rescaling algorithm ${ }^{113}$ is employed for temperature regulation at $298 \mathrm{~K}$ and the Parrinello-Rahman barostat ${ }^{114}$, 
115 is used for pressure regulation. The leapfrog integrator with a time step of $1 \mathrm{fs}$ is used to propagate the dynamics. Long-range electrostatics are treated with the smooth Particle-mesh Ewald ${ }^{116}$ method.

\section{Result and discussion}

\subsection{Recalibrating the bonded interactions.}

As has been shown in our previous work on CB8-drug systems, atomic charges could have a significant impact on the binding modes and thermodynamics. The configurational space could be explored with an initial guess with relatively low quality, and the obtained stable binding pose is used to generate a new set of atomic charges to provide more accurate descriptions of inter-molecular electrostatic interactions. As a result, the refitted atomic charges provide much better predictions of the binding thermodynamics. Further improvements of the electrostatics require more detailed models, e.g., polarizable force fields. Although most attentions of molecular modelling are attached to the charge models, the other terms could also play a crucial role in molecular recognition. Although the bonded terms are not directly involved in the calculation of intermolecular interactions, they do have impacts on the intra-molecular conformational preference of each molecule involved in host-guest interactions. Therefore, they indirectly influence the host-guest interactions and should be properly accounted for in molecular modelling. Here, we consider refitting the bonded interactions with non-bonded terms fixed to their best-performing parameters obtained in our previous work.

The generalized force-matching refitting of the parameters in the bonded interactions is performed to improve the accuracy of the model. ${ }^{99}$ The reparameterization of the bonded interactions starts from GAFF or GAFF2, and every component of the atomic forces ${ }^{117}$ and the system energy are included in the objective function. Namely, the current refitting procedure considers energy- and force-matching simultaneously. To avoid overfitting and the existence of unphysical parameters, a weak harmonic (L2) regularization term is applied to restrain the parameter space to explore in the parameter adjustment. The relative weights of the energy and force terms are the same, and that of the regularization term is 0.1 of the other two terms, leading to an intermediate level of regularization. The parameter space is further regularized according to the atomtype symmetry defined in the initial reference force field, i.e., GAFF or GAFF2. Only bonded terms including harmonic bond stretching, angle bending, and dihedral/torsion terms are refitted, and the other non-bonded terms (i.e., atomic charges and vdW terms) remain unchanged. The dihedral periodicity is also fixed in the refitting. Note that there is a special type of non-bonded interactions named the 1-4 non-bonded term. Similar to the dihedral term, this 1-4 non-bonded interaction is calculated between atoms separated by three consecutive bonds. This term is often included in the bonded terms by definition and plays an important role 
in correcting the errors of the dihedral/torsional term. However, as the atomic charges and vdW terms are kept fixed in our refitting, we do not touch the 1-4 non-bonded scaling constants and remain them fixed. In the following parts presenting the refitting details, we only discuss the re-parameterization initiated from GAFF2, while the differences between force fields refitted from GAFF and GAFF2 would be discussed in the next section.

To construct a set of independent configurations for generating reference data, for each system, we perform $110 \mathrm{~ns}$ gas-phase sampling with the sampling interval of $5 \mathrm{ps}$. A high temperature of $600 \mathrm{~K}$ is used in the unbiased simulation to enable unconstrained exploration of the configurational space. The obtained 22000 structures are used to initiate QM calculations. We have tested that this sample size or sampling time is already sufficient to converge the parameter set. Further adding samples (i.e., lengthening sampling time) leads to negligible changes in the outcomes. The selection of the target level in refitting determines the accuracy of the re-parameterized model. Here, we choose two target QM Hamiltonians. The first one is the computationally efficient semi-empirical QM (SQM) Hamiltonian of the dispersion, hydrogen-bonding, and halogen-bonding corrected PM6-D3H4X, ${ }^{118}$ which is shown to have good performance in the modelling of non-covalent interactions among SQM Hamiltonians. As for the second target Hamiltonian, we choose the ab initio level BLYP/def2-SVP ${ }^{119-122}$ with the resolution-of-the-identity approximation ${ }^{123,124}$ and the latest D4 dispersion correction $^{125,126}$ considering its good performance in modelling various properties including non-covalent interactions among pure functionals. Semi-empirical calculations are performed with the MOPAC2016 program, ${ }^{127,} 128$ while for ab initio calculations the ORCA software ${ }^{129,130}$ is employed. Note that the configuration sets for PM6-D3H4X and BLYP-D4/def2-SVP are generated independently. Thus, the parameters and errors of the refitted models are not correlated.

We first discuss the PM6-D3H4X results. To assess the quality of the refitted parameters, for the PM6D3H4X target, we generate another 25 ns trajectory (5000 configurations) at two temperatures with the newly fitted parameter set FM-PM6. The first temperature is the one that we generate the configurational ensemble in refitting, namely $600 \mathrm{~K}$. This high-temperature set enables the accuracy assessment in the ensemble that the refitting is performed. A lower temperature of $300 \mathrm{~K}$ is also employed to generate another set of configurations, which enables the assessment of energetics in the low-energy regions accessible at room temperature. Two error metrics including the root-mean-squared error (RMSE) and mean absolute error (MAE) are used for quality assessment.

In quality assessment, we first take a detailed investigation of the parameter set for the host CB8 due to its importance in coordinating all guests. In our previous work, due to the significant problem of the atomic 
charges produced by the AM1-BCC charge scheme for the host CB8 (specifically the failure of ESP reproduction), the host-guest binding affinities are generally overestimated. Thus, care should be taken when dealing with the host that is involved in all host-guest pairs. Although (S)QM Hamiltonians are more accurate than MM force fields, all of them have their own weakness in specific regions/areas. Therefore, we then crosscheck the refitted parameter sets and test whether the force field refitted targeting PM6-D3H4X is able to reproduce the energetics and atomic forces at another QM level. Here, we choose another SQM Hamiltonian of RM1. ${ }^{131}$ A similar sampling procedure is performed in this assessment, namely the 25 ns gas-phase sampling at $300 \mathrm{~K}$ to extract 5000 configurations for MM and SQM calculations. The correlations between the MM and SQM energetics when using PM6-D3H4X and RM1 as reference are provided in Fig. 2a-b, respectively. As the refitting is performed using the PM6-D3H4X data, the errors are smaller when the same SQM Hamiltonian is used as the reference level in assessment, as shown in Fig. 2a. When the reference level is set to another SQM Hamiltonian (i.e., RM1 here) different from the target PM6-D3H4X in quality assessment, interestingly, the errors of energetics are still smaller than the original GAFF2 parameters, as shown in Fig. 2b. However, the error produced by the refitted force field (i.e., FM-PM6) is obviously larger than the previous case (c.f. Fig. 2a). These observations suggest that the refitted force field targeting PM6D3H4X produces descriptions closer to a series of (S)QM Hamiltonians, not just its target level. The time series of the error of the atomic force (Frobenius norm of force) on each host atom are calculated for the PM6D3H4X reference in Fig. 2c-d with the original GAFF2 and the newly fitted parameter set, respectively. We can see that during the entire $25 \mathrm{~ns}$ sampling, the force errors are very large with the original GAFF2 parameters, and significant improvements are observed upon force-field refitting. The RMSE of the atomic forces is lowered from $40.05 \mathrm{kcal} /(\mathrm{mol} \cdot \AA \cdot$ atom $)$ to $17.87 \mathrm{kcal} /(\mathrm{mol} \cdot \AA \cdot$ atom $)$. To obtain further insights into the error distributions among atoms, the heavy atoms are sorted to the first 96 indices, and the other are hydrogen atoms. In this way, from Fig. 2c-d we can see that the force errors are significantly larger for nonhydrogen atoms. Such observation suggests that the atomic forces on heavy atoms could be more problematic than hydrogen atoms. As the conformational preference of a molecule is directly related to the rearrangement of torsional degrees of freedom involving these non-hydrogen atoms, a solution to further improving the fitting quality for atomic forces on these heavy atoms is attaching more importance to the force terms on these heavy atoms in the objective function, i.e., using larger weights for these terms. In this way, the force errors on these non-hydrogen atoms would be lowered and the conformational preference at the target level would be reproduced more accurately. Note that the force errors when selecting the RM1 Hamiltonian as the reference level have similar behaviors (results not shown). Namely, the force errors produced by the refitted force field 
are smaller than the GAFF2 results, although the reference RM1 level is not its fitting target. The observation about force errors agrees with the improvement of the system energy in Fig. $2 b$ and suggests that the refitted force field produces better descriptions of many properties closer to a series of (S)QM Hamiltonians.

The above comparison provides details that the refitted parameters of the host CB8 are more accurate than the original GAFF2 in reproducing the PM6-D3H4X energetics. We then check the statistics of all molecules. The correlation plots between the MM and SQM (PM6-D3H4X) energetics for all molecules under investigation are provided in Fig. S1. We can see that for all molecules the refitted force field produces energies closer to the $y=x$ line and thus smaller deviations from the reference level, which suggests that the refitted force field provides more accurate descriptions compared with the original GAFF2 parameter set. The time series of the deviation of atomic forces for each atom under GAFF2 and the refitted force field for the host and all guest molecules are presented in Fig. S2. We can see that for all molecules the refitted parameter set provides smaller errors for atomic forces for all atoms during the entire $25 \mathrm{~ns}$ unbiased sampling. The accuracy improvement of the refitted force field is observed at both temperatures, which indicates that the refitted force field reproduces the target PM6-D3H4X results more accurately in both the high- and low-energy regions.

The comparison between RMSEs of the energetics produced by the original GAFF2 and the newly fitted parameter set with configurations sampled at $300 \mathrm{~K}$ is provided in Fig. 2e, where smaller deviations from the PM6-D3H4X reference are observed for all molecules under the refitted parameter set FM-PM6. The RMSEs of atomic forces under the two force fields are shown in Fig. $2 \mathrm{f}$, where the overall deviations of the forces are smaller under the refitted force field. Therefore, the FM-PM6 description is closer to the target Hamiltonian PM6-D3H4X than the original GAFF2 parameter set. It is worth noting that that we have also investigated the impact of the regularization strength on the fitting outcome to avoid over- and under-regularized fitting. The regularization term used in the current refitting generally leads to an error increase of about $10 \%$, and the obtained parameters are within the reasonable range of physical properties. Overall, the refitted force field FM-PM6 provides a description much closer to the target level PM6-D3H4X than the original GAFF2 in all relevant configurational regions. Then, we turn to the other refitting target BLYP-D4/def2-SVP.

Similar procedures are repeated for the other target BLYP-D4. Namely, for each molecule, we perform $110 \mathrm{~ns}$ unbiased sampling at $600 \mathrm{~K}$ in vacuo to obtain the configurations for ab initio calculations in parameterization. As for the quality assessment, as the computational cost is much higher under the ab initio level, the sampling interval is increased to $10 \mathrm{ps}$ and the number of samples is set to 1000 . As a result, the sampling time for each molecule becomes $10 \mathrm{~ns}$ in quality assessment. The validation test (i.e., sampling and QM calculations) is only performed at $300 \mathrm{~K}$. 
Still, we first look at the CB8 results due to its involvement in all host-guest pairs. Two reference QM levels including the target BLYP-D4 and another QM level PM6-D3H4X (i.e., the previous target in the FMPM6 refitting) are used in this assessment. Note that the sets of configurations for QM calculations at the two levels are generated independently and thus the results are uncorrelated. The energetics produced by MM and QM Hamiltonians with reference to the target BLYP-D4 and PM6-D3H4X are compared in Fig. 3a-b. When the fitting target BLYP-D4 is selected as the reference level, as shown in Fig. 3a, the refitted force field provides much smaller errors for energetics than the original GAFF2. Interestingly, when the previous target PM6-D3H4X is selected as the reference level, the FM-BLYP parameter set still provides smaller errors than GAFF2, which indicates that the force field fitted targeting ab initio levels could provide descriptions not only closer to its ab initio targets but also similar to some SQM Hamiltonians. This phenomenon also suggests that the semi-empirical level PM6-D3H4X is of good quality. Another interesting comparison is between the two quality assessments in Fig. 2a and Fig. 3b. In both cases, the reference Hamiltonian is the PM6-D3H4X, but the parameter sets under assessment are FM-PM6 and FM-BLYP, respectively. The first FM-PM6 parameter set is fitted targeting the reference level, while the second FM-BLYP is not. This leads to the higher errors produced by FM-BLYP. Interestingly, the GAFF2 errors (RMSE and MAE) in the two assessments are very similar, but the latter assessment only uses 1000 configurations (1/5 of the former), which suggests that the quality assessment already converges or is bias-free at this sample size. Therefore, the 1000-configuration assessment in the FM-BLYP case is reasonable and reliable. The time series of the by-host-atom decomposition of the force errors are presented in Fig. 3c-d for the original GAFF2 and the refitted parameter set, respectively. The reference level is selected as the fitting target BLYP-D4/def2-SVP. It is clearly shown that during the entire $10 \mathrm{~ns}$ unbiased sampling, the force error on each atom is quite large with the original GAFF2, and the situation is improved significantly upon refitting. The RMSE of force errors decreases from the GAFF2 $30.06 \mathrm{kcal} /(\mathrm{mol} \cdot \AA \cdot$ atom $)$ to the FM-BLYP $13.07 \mathrm{kcal} /(\mathrm{mol} \cdot \AA \cdot$ atom). Similar to the previous FMPM6 case, the force errors are larger for heavy atoms (i.e., No. 1-96 atoms), which is expected as the limited number of degrees of freedom coupled to light atoms (hydrogen).

The above observations suggest that the refitted FM-BLYP parameter set provides descriptions closer to the target level for the host CB8. We then turn to the statistics of the guest molecules. The 1000-configuration comparisons between the MM and QM energetics for all molecules are provided in Fig. S3, where the refitted force field produces smaller deviations from the reference level for all molecules. The time series of the errors of atomic forces $\left(\left\|\Delta \mathbf{F}_{i}\right\|_{2}\right.$ for the $i$ th atom) under the original GAFF2 and the newly fitted FM-BLYP parameter 
sets are presented in Fig. S4. It is obvious that for all atoms in all molecules under investigation, the refitted FM-BLYP produces much smaller force errors than the original GAFF2.

The RMSEs of energetics produced by GAFF2 and FM-BLYP are shown in Fig. 3e, where the refitted force field produces much smaller energy deviations for all molecules under consideration. The situation of atomic forces is similar. As shown in Fig. 3f, the force errors are much smaller under the refitted force field, compared with the original GAFF2. Overall, as long as the refitting procedure is performed, the newly fitted parameter set produces descriptions closer to the target level. As the new parameter sets are constructed in a molecule-specific manner and the targets BLYP-D4 and PM6-D3H4X are of good accuracies, they are expected to provide more accurate results than the initial guess GAFF derivatives. Note that the force field refitting is also performed with another initial guess of GAFF for the PM6-D3H4X target. As the fitting procedure and quality assessment are similar to the presented cases, we would not discuss them here but focus on the differences between the behaviors of the refitted parameter sets, which would be investigated in detail in the next section.

\subsection{A closer view of different parameter sets.}

The comparisons for energetics and atomic forces presented above show that the refitted parameter sets reproduce the target Hamiltonians in a better way than the original GAFF2 parameter set. However, the detailed differences between different force fields remain unclear. As different dynamic behaviors would be produced by different parameter sets, the binding thermodynamics and interaction patterns are expected to be altered when the bonded interactions are adjusted. Therefore, before performing $\mu$ s-length enhanced sampling simulations to extensively explore the configurational space of host-guest complexes and calculate the thermodynamic landscapes, we first assess these parameter sets in detail to understand their intrinsic behaviors and the differences between them. The host CB8 is still selected as the illustrative example. As has been discussed in the system-preparation section, the vdW and bonded terms are obtained from GAFF derivatives. The difference between vdW parameters in the two GAFF force fields is small. Thus, we focus on the bonded terms.

The torsional rearrangement is the most important influencing part for conformational preferences in bonded interactions, while the other terms (i.e., bond stretching and angle bending) are relatively irrelevant. Therefore, we investigate the torsional/dihedral term in each force field in detail. The first comparison to look at is between the GAFF derivatives. Dihedral terms defined in GAFF derivatives that are used to describe the conformational preference (stiffness) of the host CB8 are shown in Fig. S5. The parameters of 
most dihedral terms in GAFF and GAFF2 for the host CB8 are similar (some are just identical), and the main difference between the GAFF and GAFF2 parameter sets lies in the torsional barrier term of the N-CN-C dihedral presented in Fig. 4a. Specifically, there is no torsional barrier explicitly defined in GAFF, while that in GAFF2 is $4.16 \mathrm{kcal} / \mathrm{mol}$. Namely, the stiffness of the CB8 ring in this region is maintained by the other dihedral terms shown in Fig. S5 in GAFF, while in GAFF2 an explicit torsional barrier is added. The phase of this dihedral is set as zero, which suggests that the addition of the torsional barrier strengthens the stiffness of the CB8 ring. Therefore, the second generation of GAFF (i.e., GAFF2) is expected to provide a stiffer CB8 ring than its precedent.

To provide further details of the dynamics of the bonded parameter sets of the CB8 host, we then perform unbiased MD simulations in explicit solvent for $20 \mathrm{~ns}$ with a sampling interval of $5 \mathrm{ps}$ with the GAFF derivatives. The superposition of snapshots obtained during MD simulations is presented in Fig. 4b-c. We can see that the CB8 ring sometimes visits the squashed conformation under GAFF even in the absence of the external guest, while under GAFF2 the host ring is quite stiff and the fluctuations of the ring are very small. These observations suggest that the GAFF2 CB8 is stiffer than the GAFF one, which is in perfect agreement with the above investigation of the detailed definitions of the torsional potential. As for refitted parameter sets, as all bonded terms are adjusted under regularized optimization, it is difficult to identify which term has the largest contribution to the different behaviors of different parameter sets. Therefore, we focus on the dynamic behaviors of these refitted force fields here. $20 \mathrm{~ns}$ unbiased simulations are performed with two newly fitted models targeting PM6-D3H4X initiated from GAFF and GAFF2 in order to investigate the influence of the initial guess in the regularized parameter adjustment. By comparing the FMPM6 parameter sets initiated from GAFF and GAFF2 in Fig. 4d-e, we know that regardless of the original parameter set used in regularization (i.e., GAFF and GAFF2), the refitted force fields provide flexible host rings. However, the shapes of the host ring under the refitted parameter sets from GAFF and GAFF2 differ. For the FM-PM6 parameter set restrained around GAFF, the host cavity seems like a cycle. By contrast, a rectangular cavity is maintained by the FM-PM6 parameters restrained around GAFF2. The RMSEs of energy and atomic forces of the FM-PM6-from-GAFF and FM-PM6-from-GAFF2 parameter sets are similar (results for the former not shown), which suggests that the two parameter sets provide descriptions with similar sizes of errors/deviations from the target level. Thus, it is difficult to say which force field is more accurate. As the GAFF force field has been employed in extensive sampling simulations in our previous work, ${ }^{83}$ in the current work we use the GAFF2 parameter set in simulations and as the initial guess used to initiate the regularized parameter optimization. Generally speaking, the BLYP-D4 level is more accurate 
than PM6-D3H4X and the refitted parameter set targeting BLYP-D4 is of higher reliability. Also considering the system-specific fitting parameters and the resulting broken transferability, the refitted FM-BLYP parameter set is expected to be the best (most accurate) description employed in the current work. The superposed snapshots obtained under FM-BLYP-D4 from GAFF2 are presented in Fig. 4f, where a different behavior of the CB8 ring is observed. The refitted FM-BLYP parameter set provides a stiff host ring similar to the GAFF2 description. As this target level is more accurate than PM6-D3H4X, this stiffer behavior of the host cavity is considered to be more reliable. Based on the above observations of the host ring in plain explicit-solvent simulations, we expect the CB8 ring to be stiff and maintain its perfect-circle conformation in the absence of the guest. On this aspect, GAFF2 and FM-BLYP perform well, FM-PM6 is of intermediate quality, and GAFF performs worst. However, we cannot guarantee that this accuracy rank is applicable to host-guest complexes in solvent, where complex inter-molecular interactions are involved. According to the observations about the CB8-drug systems in our previous work based on GAFF, ${ }^{83}$ upon host-guest binding and the formation of strong inter-molecular interactions, the host ring could possibly be distorted and squashed to hold the guest tightly. In that case, a too stiff description of the host ring could trigger the inability of the host to squash and fit the shape of the binding guest in the bound state, leading to inappropriately looser host-guest coordination.

The radius of gyration $(\mathrm{Rg})$ is often chosen as the $\mathrm{CV}$ to describe the open and close of the host cavity. However, the behavior of this $\mathrm{CV}$ is generally not investigated in detail and thus the correctness of this selection is not justified. To analyze the detailed behavior of this $\mathrm{CV}$, we compare the time series of $\mathrm{Rg}$ of the host ring under GAFF2 and the refitted force fields in Fig. 4g. The GAFF2 curve is quite stable, which is in agreement with the stiffness of the host ring produced by this parameter set. By contrast, the Rg curves for the refitted FM-PM6 force fields initiated from GAFF and GAFF2 experience significant fluctuations, which suggests the dynamical behaviors of the host ring and the existence of an ensemble of host conformation. It is worth noting that the maximum and minimum values for the two FM-PM6 force fields are similar, and their fluctuation behaviors also show similar trends. However, the detailed structural features produced by the two refitted force fields differ, as shown in the above superposed snapshots (c.f., Fig. 4e-f). Therefore, this CV (i.e. Rg) cannot properly differentiate different structural features produced by different force fields and thus may not be a good CV to analyze the detailed host conformation. The FM-BLYP parameter set provides a stable Rg curve and higher values of Rg than the original GAFF2 parameter set, which suggests that the host cavity described with this parameter set is wider than GAFF2. Overall, FMBLYP and GAFF2 behave similarly and describe a stiff host ring with a wide central cavity, while the host 
rings described by FM-PM6 and GAFF are of higher flexibilities.

In this section, it has been observed that the employed GAFF derivatives and refitted parameter sets produce different behaviors of the host cavity in the decoupled/unbound state. In the next section, the thermodynamic behaviors of the employed parameter sets in the coupled/bound state would be investigated in detail.

\subsection{Binding modes.}

In host-guest and more generally protein-ligand modelling, the most influencing part in classical force fields is considered to be electrostatics. As the electrostatic interaction is the main contributor of intermolecular non-bonded interactions, the atomic charges are expected to have significant impacts on the binding thermodynamics and interaction patterns. Due to the low transferability of atomic charges for druglike molecules, this term is often refitted in a case-by-case manner to provide accurate descriptions. In our previous extensive computational investigation of CB8-drug binding, the GAFF parameters are used to describe intra-molecular bonded interactions and the electrostatics are described with AM1-BCC and RESP charges. Different charge schemes lead to different features of the $\rho-C$ and $C-C_{P h}$ free energy surfaces. The best combination in the previous work is considered to be GAFF+RESP, considering the ESP reproduction and the accuracy of binding thermodynamics.

Although the bonded interactions are not directly involved in the calculation of inter-molecular interactions, they do significantly influence the inter-molecular interactions. This indirect influence is achieved by determining the conformational preference of molecules involved in inter-molecular interactions. Specifically, the distances between different components involved in inter-molecular interactions are different under different force fields, which leads to different strengths of charge-charge interactions and thus different inter-molecular interaction patterns. Therefore, considering the significance of the bonded terms, the focus of the current work is comparing and refitting bonded interactions. Different parameter sets could lead to different favorable regions in the coupled and decoupled states.

The general picture of CB8-guest interactions observed in our previous extensive modelling is a squashed CB8 ring that holds the guest tightly. ${ }^{83}$ Intuitively, such squashed host ring is energetically unfavorable under the stiff GAFF2 parameter set and thus the GAFF2 binding modes are expected to show some differences compared with the GAFF ones observed in our previous work. The re-parameterized set FM-PM6 provides a host cavity with intermediate levels of flexibility and thus is expected to have different behaviors compared with the GAFF derivatives. As the host behavior under the FM-BLYP parameter set is 
similar to the initial guess GAFF2, this refitted parameter set is expected to have similar behaviors to GAFF2. Aside from the structural features of the host cavity, the behaviors of the guests are also altered when changing the bonded parameter set. The alternations of the host and the guest would lead to different intra- and inter-molecular interaction patterns. In the following paragraphs, we would investigate whether the previously observed general picture of CB8 host-guest binding is still valid when different bonded parameter sets are employed.

In the analysis of the host-guest coordination, we still start by monitoring the total host-guest contacts and its by-host-atom decomposition to check whether the space of the relative host-guest position is properly explored. The illustrative example chosen here is the complex formed by CB8 and the deprotonated G5 described by the FM-PM6 parameter set, as shown in Fig. 5. Note that all atoms of the host and the guest are included in this calculation, while in the free energy analysis shown later only heavy atoms are considered. In Fig. 5a, during the 1000 ns enhanced sampling simulation, the total host-guest contact number experiences significant fluctuations, which suggests that the exploration of the space of the relative hostguest position is accelerated. The total contact number reaches its maximum around 1800 and approaches zero many times, which indicates that the bound and unbound states are reached many times. More details about the host-guest coordination could be obtained from the by-atom decomposition shown in Fig. 5b. For instance, in the cyan oval, the atom-guest contacts are larger than 1 for all host atoms, which indicates that all host atoms are in direct contacts with the guest. The total host-guest contact number in this region is close to the maximum of the observed values. These phenomena suggest a typical center-binding pose, where the whole guest stays at the center of the host cavity. Another example is presented in the magenta oval. The total contact number in this region is obviously lower than the maximum value but is much larger than zero. This intermediate value suggests a looser host-guest coordination. More detailed coordination patterns could be extracted from the atom-guest contacts, where only some host atoms have non-zero atom-guest contacts. Therefore, the host-guest coordination picture in the magenta oval is that the guest binds to the outer surface of the host ring, which corresponds to a side binding pose. Similar observations could be obtained from the time series for all host-guest pairs under GAFF2, FM-PM6 and FM-BLYP presented in Fig. S6-S8. Therefore, the sampling does explore the space of host-guest coordination effectively. We then turn to the thermodynamic landscapes to investigate the stability of these explored interaction patterns.

We start by checking the GAFF2 results. The radius-contact $(\rho-C)$ surfaces under this force field are presented in Fig. S9. For the guest G1, only one wide free energy minimum is observed. The bound conformation extracted there features the whole guest fluctuating inside the host cavity and the formation of 
inter-molecular $-\mathrm{NH}_{2}{ }^{+} \cdots \mathrm{O}=\mathrm{C}$ - hydrogen bonds, which is similar to the GAFF case observed in our previous work. ${ }^{83}$ As for the structurally complex guest G2, there are generally three bound conformations under GAFF2. The two small- $C$ minima with higher thermodynamic stabilities feature the phenyl ring connected to the amide bond staying at the center of the host and the extended guest clinging to one side of the host, and their differences mainly lie in the degree of distortion to the CB8 ring. The other large- $C$ minimum is less stable, and its larger host-guest contact number arises from the fact that the middle part of the guest is coordinated at the host center and the two phenyl rings are outside the cavity (i.e. solvent-exposed). All these conformations are observed and of high thermodynamic stabilities under GAFF. Thus, the patterns of the host-guest coordination are not significantly different under the GAFF derivatives. For the structurally rigid guest G3, similar to the GAFF case, we only observe one narrow free energy minimum, the structural features of which include the center-binding behavior and the inter-molecular hydrogen bonds formed between the $-\mathrm{NH}^{+}$and the two $-\mathrm{OH}$ groups of the guest and the $-\mathrm{C}=\mathrm{O}$ portals of the host. The free energy surface for the CB8-G4 complex is altered compared with the previous GAFF result. The bound state becomes less stable when replacing GAFF with GAFF2, and the host-guest interaction is weaker. The most stable bound conformation locates at the largest- $C$ minimum, which depicts a center-binding pose with $\mathrm{NH}^{+}$and $-\mathrm{OH}$ of the guest forming hydrogen bonds with the $-\mathrm{C}=\mathrm{O}$ portals of the host. When the host-guest COM distance grows, the guest gradually leaves the cavity center. There are two wide free energy minima along the dissociation pathway. In the first minimum with larger host-guest contacts, the guest starts to leave the host center but the inter-molecular hydrogen bonds are still formed between $-\mathrm{NH}^{+}$and $-\mathrm{OH}$ and the $\mathrm{C}=\mathrm{O}$ portals. When the host-guest $\mathrm{COM}$ distance further increases, the system reaches the second minimum, where only the $-\mathrm{NH}^{+} \cdots-\mathrm{C}=\mathrm{O}$ hydrogen bond remains. The protonated and deprotonated forms of $\mathrm{G} 5$ have similar radius-contact surfaces. Center-binding poses with the whole guest fluctuating inside the host cavity are observed in their bound conformations, which is still similar to the GAFF case. The situation is similar for the guest G6, where center-binding poses with many host-guest coordination patterns are observed. As for the last guest G7, still many interaction patterns could be observed. For instance, in the center-binding pose, the phenyl ring could be in or outside the central cavity. Therefore, the radius-contact surfaces obtained under GAFF2 are similar to the GAFF results.

We then turn to the first refitted parameter set FM-PM6. The radius-contact surfaces are presented in Fig. S10. For the structurally simple guest G1, the bound conformation still features the guest fluctuating inside the host cavity. However, compared with the GAFF2 case, the host cavity is more flexible. The rectangular host cavity observed in the unbound state (c.f., Fig. 4e) is still thermodynamically stable in the 
bound state, but many other shapes are also favorable. The presence of these irregularly shaped host rings suggests that the inter-molecular host-guest interactions could alter the dynamic behavior of the host, and insights obtained in host-only or guest-only simulations could be limited. Note that the change of the bonded parameters for the guest G1 also plays a role in the alternation of host-guest binding. The free energy surface of the structurally complex G2 under FM-PM6 also shows some differences compared with GAFF2. There are one wide large-distance and one narrow small-distance minima, both of which are center-binding poses that have been observed under GAFF2. The two narrow large-distance minima under GAFF2 are merged into the wide large-distance minimum under FM-PM6, and the FM-PM6 minimum becomes further wider due to the increased flexibility of the host ring under this parameter set. As for the small-distance minimum under GAFF2, it becomes thermodynamically stable under FM-PM6 also due to the increased flexibility of the host ring. The wide large-distance minimum contains many host-guest coordination patterns. When comparing the bound conformations extracted from the small-distance minimum and the region with similar host-guest contacts in the large-distance minimum, some structural features unique in each conformational state could be identified. For instance, two $-\mathrm{CH}_{2}$ - groups of the host rotate into the inner side of the cavity but show different behaviors in the two conformational states. In the large-distance minimum, these two groups are symmetric on the host ring, i.e., in the $i$ th and $(i+4)$ th repeating units, which leads to a relatively symmetric host cavity. As the guest is clinging to one side of the host (i.e., asymmetric binding), the COMs of the host and the guest are separated relatively far. By contrast, in the small-distance minimum, the two $\mathrm{CH}_{2}$ - groups are distributed on the $i$ th and $(i+3)$ th repeating units, which leads to a severely asymmetric host cavity. The asymmetric binding of the guest to the host ring and the asymmetric orientation of the $-\mathrm{CH}_{2}$ groups leads to tighter host-guest coordinations and thus a smaller COM distance. Similar to the cases of the GAFF derivatives, the radius-contact free energy surface for the structurally rigid G3 under FM-PM6 only has one narrow free energy minimum, which suggests that the number of favorable host-guest coordination patterns is limited. In the center-binding pose, the $-\mathrm{NH}^{+}$and $2-\mathrm{OH}$ groups of the guest form inter-molecular hydrogen bonds with the $-\mathrm{C}=\mathrm{O}$ portals of the host. However, the shape of the host cavity is quite irregular, which is in agreement with the increased host flexibility observed in unbiased simulations. Therefore, we can see that the bonded parameters could have significant impacts on the host-guest coordination pattern. As for the structurally rigid G4, two free energy minima are observed on the radius-contact surfaces, which is similar to the GAFF2 case. The first large-C center-binding pose features the $-\mathrm{NH}^{+}$and -OH groups of the guest forming inter-molecular hydrogen bonds with the $-\mathrm{C}=\mathrm{O}$ portals of the irregularly shaped host, while the second large-distance minimum has some parts of the guest leaving the host cavity. As for the protonated 
G5, the only free energy minimum observed is quite wide, which is different from the previous GAFF2 case. The inter-molecular hydrogen bond is still formed between the $-\mathrm{NH}_{2}{ }^{+}$group of the guest and one of the $\mathrm{C}=\mathrm{O}$ portals of the host, and the guest fluctuates inside the host cavity in the center-binding mode. However, the host cavity is quite flexible under FM-PM6. The simultaneous conformational fluctuations of the irregularly shaped host and the guest make the host-guest coordination flexible. The situation is similar for the deprotonated G5. Namely, the narrow GAFF2 minimum becomes wider when shifting to FM-PM6, due to the increased flexibility of the host cavity. For the structurally complex G6, there is only one free energy minimum on the radius-contact surface. Similar to the GAFF2 result, this minimum is narrow and still features center-binding. As for the last guest G7, two free energy minima are observed on the radius-contact surface. The bound conformations extracted from these minima feature the center-binding behavior with many interaction patterns. For instance, the phenyl ring of the guest could stay in or outside the host cavity. The shape of the host is still irregular. When comparing the FM-PM6 surface with the GAFF2 one, we know that the wide minimum containing a variety of binding poses under GAFF2 is split into the two sub-minima under FM-PM6. The refitted FM-PM6 CB8 ring could alter its conformation to tightly coordinate the guest, while under GAFF2 the ring conformation remains almost unchanged. Note that the refitting of the bonded parameters of the guest also plays a role here. Overall, the FM-PM6 parameter set provides similar descriptions of host-guest coordination to the GAFF2 case, but the detailed interaction patterns differ in many aspects.

We finally investigate the last bonded parameter set FM-BLYP. The radius-contact surfaces under this parameter set are shown in Fig. S11. As shown in the unbiased simulations under different bonded parameter sets, the host behaviors under the FM-BLYP parameter set are similar to those under GAFF2. Thus, we expect the host conformation to be similar to the GAFF2 case in host-guest coordination. However, as the refitting procedure also alters the description of the guests, the conformational preference, the coordination pattern and the strength of inter-molecular interactions are expected to be altered. For the structurally simple guest G1, a wide free energy minimum is observed. The guest fluctuates inside the central cavity and forms inter-molecular hydrogen bonds with the $-\mathrm{C}=\mathrm{O}$ portals of the host. Similar to GAFF2, the host ring is less distorted compared with the FM-PM6 case. As for the structurally complex G2, the host-guest binding pattern is still the extended guest clinging to one side of the host. The low-(free-)energy regions are similar to the previous cases. From these free energy minima, many binding poses could be observed. For example, the middle part of the guest can stay at the host center and its $-\mathrm{NH}^{+}$forms a hydrogen bond with one $-\mathrm{C}=\mathrm{O}$ portal of the host. The G3 case is still similar to the previous cases. In the only free energy minimum, the 
guest binds to the center of the host with its $-\mathrm{NH}^{+}$and $2-\mathrm{OH}$ groups forming inter-molecular hydrogen bonds with the $-\mathrm{C}=\mathrm{O}$ portals of the host. The structurally rigid $\mathrm{G} 4$ under FM-BLYP only has one free energy minimum, where the center-binding pose is observed and its $-\mathrm{NH}^{+}$and $-\mathrm{OH}$ groups form hydrogen bonds with the $-\mathrm{C}=\mathrm{O}$ portals of the host. This difference between the FM-BLYP and GAFF2 results mainly arises from the change of the intra-molecular interactions of the guest G4, which suggests that only monitoring the behavior of the host dynamics could lead to limited insights about inter-molecular coordination and the system-specific refitting of the bonded terms to improve the accuracy of the description is necessary even for structurally rigid guests. As for the protonated G5, only one free energy minimum is observed on the radius-contact surface. The FM-BLYP minimum is wider than the GAFF2 one but is narrower than the FMPM6 case. The most stable bound conformation has the whole guest inside the host cavity, and conformational fluctuations would cause some parts of the guest to leave the host center and become solvent-exposed. The situation is similar for the deprotonated G5. Specifically, there is only one minimum observed on the radius-contact surface with the guest fluctuating inside the host cavity. Two free energy minima are observed for the guest G6. The large-C minimum is more thermodynamically stable than the other one. The guest binds at the center of the host, and conformational fluctuations would lead to some parts of the guest leaving the host cavity, producing less compact bound conformations. Note that this twominima behavior differs from the GAFF2 and FM-PM6 results, which again suggests that although the GAFF2 and FM-BLYP parameter sets produce similar host dynamics, the alternation of the guest description would lead to obvious differences in inter-molecular coordination. A wide free energy minimum is observed for the last guest G7. This observation is similar to the GAFF2 case, but this wide minimum is cut into two narrower ones under FM-PM6. The wideness of this bound state indicates the existence of many host-guest binding patterns.

As observed in our previous work and in the current results, under all Hamiltonians (force fields) the combination of the COM distance $\rho$ and the host-guest contacts $C_{\text {host-guest }}$ cannot properly differentiate different interaction patterns. Thus, we then consider the $C_{\text {host-guest }}-C_{\text {aromatic }}$ or $C-C_{P h}$ surfaces, as done in our previous work. ${ }^{83}$ There is at least one aromatic ring formed by six carbon atoms in each host, and the contact number between the aromatic 6-membered ring of the guest and the host is used to decompose the overall host-guest coordination into the host-aromatic and the other ones. We still only consider the heavy atoms in this free energy analysis. As there are two phenyl rings for Fentanyl G2, we select the phenyl ring directly connected to the amide bond. We again note that the current analyses of host-guest binding patterns 
under different bonded parameter sets are strongly related to the discussions about GAFF binding poses reported in our previous work. Thus, we highly recommend the readers to refer to the previous paper for a thorough understanding of the current results and discussions.

In the current $C-C_{P h}$ analysis, we still first check the GAFF2 results shown in Fig. 6. For the structurally simplest guest under study, G1, a single wide free energy minimum is observed on the free energy surface. Structures extracted there share the center-binding feature with inter-molecular hydrogen bonds formed between $-\mathrm{NH}_{2}{ }^{+}$of the guest and the $-\mathrm{C}=\mathrm{O}$ portals of the host. The host cavity is less distorted compared with the GAFF case, which is related to the higher stiffness of the GAFF2 description. The free energy surface for the structurally complex G2 shows obvious differences compared with the GAFF result. Under GAFF2, the binding poses with large host-phenyl contacts are no longer stable, and only conformational states with small $C_{P h}$ are favorable. On the free energy surface, the most stable binding pose lies in the state with the smallest host-phenyl contacts. In this conformational state, the phenyl ring on the $-\mathrm{NH}^{+}$side penetrates the cavity, the amide bond part of the guest clings to one side of the host ring, and the phenyl connected to the amide bond stays away from the host. The inter-molecular $-\mathrm{NH}^{+} \cdots-\mathrm{C}=\mathrm{O}$ distance is longer than the normal criterion for hydrogen bonds, suggesting the absence of host-guest hydrogen bonds. It is worth noting that compared with the GAFF case where the host ring is squashed significantly in host-guest coordination, under GAFF2 the host ring is perturbed at a minimal level, which is expected due to the higher host flexibility defined by the GAFF parameters discussed extensively in the previous section. The minimum next to the first minimum has larger host-guest and host-phenyl contacts. This conformation is of the highest stability under GAFF, but under GAFF2 it is about $0.5 \mathrm{kcal} / \mathrm{mol}$ less stable than the previous minimum. In this binding pose, the guest nestles against the host with the phenyl moiety connected to the amide bond binding to the outer surface of the host, while the other phenyl ring stays at the center of the host. Still, no inter-molecular $-\mathrm{NH}^{+} \cdots-\mathrm{C}=\mathrm{O}$ hydrogen bond is formed. The differences between the GAFF and GAFF2 results suggest that different behaviors of intra-molecular terms could lead to different inter-molecular binding thermodynamics and interaction patterns in host-guest complexes. The CB8-G3 surface under GAFF2 is rather simple. For this structurally rigid guest, the only bound conformation features the whole guest inside the central cavity and is similar to the GAFF case. The $\mathrm{NH}^{+}$and two $-\mathrm{OH}$ groups of the guest form hydrogen bonds with the $-\mathrm{C}=\mathrm{O}$ portals of the host. Although $\mathrm{G} 4$ is very similar to G3, the free energy surface of CB8-G4 shows more complex behaviors. The typical centerbinding conformation is no longer the dominant binding pose in the host-guest coordination, but there are a 
series of stable binding patterns even when the inter-molecular coordination is not very compact. There are two states with obviously larger host-guest and host-phenyl contacts than the other states. These two largecontact states belong to variations of the center-binding poses, while the other could be grouped into intermediate states along the binding/unbinding pathway. The most stable bound state has the largest $C$ but an intermediate value of $C_{P h}$. In this state, the $-\mathrm{OH}$ group of the guest forms a hydrogen bond with a $-\mathrm{C}=\mathrm{O}$ portal, but the $-\mathrm{NH}^{+}$group is perpendicular to the host ring. The aromatic ring of the guest is relatively far from the host center compared with the other large- $C$ pose, which is expected considering its smaller $C_{P h}$. Compared with the global minimum, the other center-binding pose has similar host-guest contacts but larger host-aromatic (phenyl) contacts. Thus, we expect that in this pose the guest still stays at the host center but its aromatic ring would be closer to the host center. Visualizing the structure extracted there, we confirm that the guest is indeed inside the host cavity but its orientation differs from the first pose. The aromatic moiety of the guest is encapsulated at the center of the host cavity while the other parts of the guest are relatively solvent-exposed. When the host-guest and host-phenyl contacts begin to fall, the guest starts to leave the host center. The first free energy basin on this association/dissociation pathway next to the global minimum features a larger distance between the aromatic ring and the host center and the inter-molecular hydrogen bonds formed between $-\mathrm{OH}$ and $-\mathrm{C}=\mathrm{O}$ portals. When the contact numbers drop further, two host-guest coordination patterns are reached. In the conformational state with larger $C$ but smaller $C_{P h}$, the guest becomes further farther from the host center and most parts of the guest are in solvent. Still, this binding pose has the basic center-binding features and can be considered as a pre-formed center-binding pose. However, the other pose with smaller $C$ but larger $C_{P h}$ has a totally different inter-molecular coordination pattern. At this position, the guest binds to the outer surface of the squashed host, which makes it a sidebinding pose that is very unstable under GAFF. Overall, for the CB8-G4 complex, the GAFF2 description produces a variety of host-guest coordination patterns instead of the single center-binding preference under GAFF. The protonated and deprotonated G5 under GAFF2 show similar behaviors to the GAFF cases. The crescent free energy landscapes depicting the conformational fluctuations of the guest inside the host cavity are still observable, and minor differences between the GAFF and GAFF2 results lie in the detailed distributions of the low-(free-)energy regions. The protonated G5 has a narrower low-(free-)energy minimum than the deprotonated form. The bound structure extracted at the center of this minimum has the typical center-binding features, which is very similar to the GAFF result. As for the deprotonated G5, its free energy surface is relatively rugged and the low-(free-)energy minima are distributed sparsely. We extract 
four structures on the surface to analyze the detailed host-guest coordination patterns. The binding pose at the center of these minima is the typical center-binding one, where the whole guest is inside the host cavity. The other two minima on the crescent and in the neighborhood of the global minimum are related to the conformational fluctuations of the guest inside the host cavity. These binding poses are produced when one of the two 6-membered rings of the guest leaves the host center. The above observations about the protonated and deprotonated forms of G5 are similar to the GAFF results, but there is one new stable binding pose for the GAFF2 deprotonated G5. In the pose next to the typical center-binding position and with larger host-guest and host-phenyl contacts, the host ring becomes more squashed and the host-guest coordination becomes tighter than the other conformational states. The -NH group of the guest forms one hydrogen bond with a $-\mathrm{C}=\mathrm{O}$ portal. This binding conformation is observed under GAFF but is not as stable as the typical minimum. However, under GAFF2 this binding pose is of similar thermodynamic stability to the other. This phenomenon suggests that the conformational-fluctuation picture is also dependent on the bonded parameters. The structurally complex G6 also produces significantly different host-guest coordination patterns under the two GAFF derivatives. Under GAFF, two well-separated minima are observed, and the host-guest coordination is considered to be dominated by two interaction patterns. ${ }^{83} \mathrm{By}$ contrast, an extremely wide free energy basin containing a series of bound conformations is observed under GAFF2. This phenomenon is somehow similar to the G5 case, where a wide crescent free energy landscape that is highly related to the conformational fluctuation of the guest inside the host cavity is observed. As a result, the CB8-G6 coordination is considered to be similar to the G5 one. Namely, the host-guest coordination is highly dynamical and the conformational fluctuation of G6 inside the host cavity is significant. To understand the details of the host-guest binding pattern, we basically extract three representative structures from three distant positions on the free energy surface. The most stable binding pose or the global minimum lies at the center of this wide free energy basin. The structure at this position features the phenyl ring outside and the other two 6-membered rings inside the host cavity. The $-\mathrm{NH}^{+}$group of the guest is perpendicular to the host ring and thus no inter-molecular hydrogen bond is formed. The other two thermodynamically less stable poses are extracted from the tails of the wide free energy basin. In the pose with larger host-guest and host-phenyl contacts, the piperidinium and phenyl moieties penetrate the host cavity and the cyclohexyl ring is solvent-exposed. $-\mathrm{NH}^{+}$is still almost perpendicular to the host ring, which makes it impossible for the formation of intermolecular hydrogen bonds. In the other one with smaller host-guest and host-phenyl contacts, both the phenyl and cyclohexyl moieties flip out and only the piperidinium ring stays inside the host cavity. From the above structural information, we can summarize the 
basic feature of the conformational fluctuation inside this wide free energy basin. The common feature of these three binding structures is that the piperidinium ring is always inside the host cavity or close to the host center, while one of the other two 6-membered moieties (i.e., phenyl and cyclohexyl) of both of them can flip outside the host cavity without causing significant unfavorable interactions. Another worth noting point observed on the free energy surface is that the wide free energy minimum under GAFF2 covers almost all energetically favorable regions under GAFF, which suggests that the GAFF binding poses are a subset of the GAFF2 one. This conclusion can also be reached by comparing the host-guest binding patterns reported in our previous work and the current GAFF2 results. The last guest under investigation, G7 Cocaine, is also found to have different behaviors under the two GAFF derivatives. Unlike the GAFF case where only a single bound conformation is preferred, under GAFF2 the CB8-G7 complex has a very complex thermodynamic landscape with multiple low-(free-)energy minima. There are basically four conformational states on the $C-C_{P h}$ surface. The most obvious difference between these states that can be identified on the free energy surface is $C_{P h}$. The global minimum or the most stable binding pose has the largest hostphenyl contacts, which suggests a tight host-phenyl coordination at this position. In this state, only the phenyl ring is penetrating the squashed host cavity, while the other parts are far from the host center and solvent-exposed. No inter-molecular hydrogen bond is formed between the host and the guest. Note that this conformational state is also the most stable one under GAFF, and the detailed host-guest interaction patterns at this position are similar under the two GAFF derivatives. Thus, the most stable binding poses are similar under the GAFF derivatives. However, when $C_{P h}$ drops down and the system leaves the minimum, the thermodynamic stability of the host-guest complex decreases dramatically under GAFF, while under GAFF2 a series of (meta-)stable conformations with relatively high stabilities are reached, which depicts a multistate picture for host-guest coordination instead of the single-pose-dominant one under GAFF. In the smaller- $C_{P h}$ state next to the global minimum, the phenyl ring inserts deeper into the host cavity and comes out from the other side, and the other parts of the guest are thus closer to the host center, which leads to smaller host-phenyl contacts and larger host-guest contacts. The existence of this binding pose is obviously due to the conformational fluctuations of the guest inside the host cavity. Note that this binding pose is observed under GAFF, but its thermodynamic stability is much lower than the first pose in that case. By contrast, this conformational-fluctuation-induced binding pose is of similar thermodynamic stabilities to the first binding pose under GAFF2. Thus, it is obvious that the conformational preferences produced by the 
GAFF derivatives are somehow different. When $C_{P h}$ drops further, the phenyl moiety becomes further away from the host center. The low-(free-)energy pathway on the free energy surface for this process suggests an increase of host-guest contacts, which indicates that the other parts of the guest become closer to the host center. The structure visualization confirms this host-guest coordination picture. We can see that only the phenyl moiety is outside the host cavity, while all the other parts of the guest stay at the center of the host ring. Obviously, this binding pose is also highly related to the previous two poses through conformational fluctuations. However, unlike the previous two poses, the free energy barrier between this binding pose and the other two is high, which suggests that the interconversion between them is not easy. The $4^{\text {th }}$ pose has smaller host-guest and host-phenyl contacts compared with the $3^{\text {rd }}$, which draws a picture that all parts of the guest start to leave the host center. The structure extracted here shows that the phenyl ring becomes further away from the host cavity, and the other parts of the guest are also leaving from the host center. This seems like an intermediate when the host-guest complex starts unbinding. The above GAFF2 $C-C_{P h}$ surfaces show obvious differences from the GAFF results in our previous work in many aspects, which suggests that the inter-molecular coordination pattern is strongly dependent on the intermolecular interaction terms. For the host cavity, the GAFF2 parameter set differs from GAFF mainly in the stiffness of the host ring, while the parameters for the guest molecules are also changed when we shift from GAFF to GAFF2. These factors, as a whole, trigger the somehow different CB8-drug binding patterns, but the general picture of the guest dynamically coordinated by a distorted host with significant conformational fluctuations remains unchanged.

We then turn to the molecule-specific FM-PM6 parameter set. It has been observed in unbiased simulations that compared with the initial guess GAFF2, this refitted parameter set produces a more flexible host cavity. Thus, we expect to observe a flexible host in the bound state. Aside from the host behavior, as the guest descriptions are also altered upon force-field refitting, the host-guest coordination is expected to be changed also in many other aspects. The $C-C_{P h}$ free energy surfaces under this parameter set are presented in Fig. 7, and the visualization of structures is used to aid the understanding of the detailed intermolecular coordination pattern. For the structurally simple guest G1, we observe a single free energy minimum that is much wider than the GAFF2 case. To understand the structural features in this wide conformational state, we extract three structures from the center of this minimum and its large-contacts and small-contacts tails. The system is of the highest stability when the two contact numbers are large, and the structure on the right depicts a coordination pattern that the whole guest penetrates the host cavity, the host 
ring becomes squashed to maximize the host-guest coordination, and the $-\mathrm{NH}_{2}{ }^{+}$group of the guest forms inter-molecular hydrogen bonds with the $-\mathrm{C}=\mathrm{O}$ portals of the host. In the binding pose at the middle that is about $1 \mathrm{kcal} / \mathrm{mol}$ less stable, the guest still stays in the host cavity but is more extended than the previous pose. The host is squashed but the host-guest coordination is not as tight as the previous pose. Intermolecular hydrogen bonds are formed between the $-\mathrm{NH}_{2}{ }^{+}$group of the guest and the $-\mathrm{C}=\mathrm{O}$ portals of the host. The structure at the left has smaller host-guest and host-phenyl contacts than the other two, which suggests a looser level of inter-molecular coordination and a larger host-guest distance. As expected, in the left structure we observe that the phenyl ring of the guest still penetrates the host cavity, but the other parts become relatively far from the host center. The inter-molecular hydrogen bonds are still formed. Overall, for the CB8-G1 binding under FM-PM6, the host cavity in the bound state is more squashed and irregularly shaped than the GAFF2 case, which is in agreement with the increased flexibility observed in unbiased simulations. As for the structurally complex G2, the FM-PM6 surface also shows obvious differences compared with the initial guess GAFF2. Generally, there are four conformational states observed on the $C-C_{P h}$ surface, which is more similar to the GAFF case instead of the initial guess GAFF2. This phenomenon is in agreement with the flexibility of the host cavity observed in unbiased simulations under this newly fitted parameter set, which is more similar to GAFF than GAFF2. The positions of the observed four conformational states are also very similar to those observed under GAFF. However, by visualizing the structural details of the bound conformations, we find that the FM-PM6 binding poses show obvious differences with the GAFF ones. The four conformational states could be divided by their host-phenyl contacts. The two minima on the top-right have larger host-phenyl contacts, and their difference lies in the host-guest contacts, which suggests that the phenyl ring is in close contact with the host ring while the other parts of the guest fluctuate and produce different conformational features. In the state with the larger hostguest contacts, a very typical center-binding pose is observed. The whole guest is in the CB8 cavity and the guest is folded in a compact form to insert tightly into the host cavity. The $-\mathrm{NH}^{+}$group of the guest forms a hydrogen bond with one of the $-\mathrm{C}=\mathrm{O}$ portals of the host. In the other state with smaller host-guest contacts, the whole guest clings to the host and phenyl at the amide bond side penetrates the host. No inter-molecular hydrogen bond is formed. This conformational state is less stable than the first one, which is in accordance with the GAFF results. Note that these two large- $C_{P h}$ states are thermodynamically unstable under the initial guess GAFF2, but are favorable under the more flexible GAFF. However, under either GAFF or GAFF2, the host cavity is not as twisted as the current FM-PM6 case when coordinating the penetrating 
groups. The other two states with smaller host-phenyl contacts also have smaller host-guest contacts, and their difference mainly lies in the host-phenyl contacts. In the state with larger host-phenyl contacts, the basic picture of host-guest coordination under FM-PM6 is still similar to those observed under the GAFF derivatives. Specifically, the guest nestles against the host with the phenyl connected to the amide bond binding to the outer surface of the host, while the other phenyl ring stays at the center of the host. However, it is observed again that under either GAFF or GAFF2, the host cavity is not so twisted as the current FMPM6 case. In the last state with the smallest host-guest and host-phenyl contacts on the free energy surface, the basic features of this binding pose are also similar to the results under the GAFF derivatives. Namely, the phenyl ring on the $-\mathrm{NH}^{+}$side penetrates the cavity, the middle part of the guest clings to one side of the host ring and the selected phenyl ring stays away from the host. The inter-molecular $-\mathrm{NH}^{+} \cdots-\mathrm{C}=\mathrm{O}$ hydrogen bond seems to be formed. However, the host ring is more distorted than the GAFF and GAFF2 cases. Overall, during the CB8-G2 interactions, the basic features of the interaction patterns remain unchanged, but the host cavity is more twisted than the previous GAFF and GAFF2 cases, which is in agreement with the observations in the CB8-G1 complex. As for the structurally rigid G3, only one conformational state is observed on the $C-C_{P h}$ surface, which agrees well with the GAFF derivatives. This center-binding pose feature the whole guest inside the central cavity with its $-\mathrm{NH}^{+}$and two $-\mathrm{OH}$ groups forming hydrogen bonds with the $-\mathrm{C}=\mathrm{O}$ portals of the host. However, the host ring is more twisted and irregularly shaped compared with the other parameter sets, which is expected considering the dynamical behavior of the CB8 ring in unbiased simulations reported in the previous section. The guest G4 is structurally similar to G3, but this time under FM-PM6 it still has an obviously different $C-C_{P h}$ surface compared with G3. The centerbinding minimum with large host-guest and host-phenyl contacts is wider than the GAFF or GAFF2 case, which suggests a higher level of conformational fluctuations. Two microstates could be identified in this large- $C$ (or equivalently large- $C_{P h}$ ) basin. The most stable binding pose lies in the minimum with the largest host-guest contacts. It depicts a typical center-binding pose with the inter-molecular host-guest hydrogen bonds formed and the aromatic ring of the guest staying at the host center. The other microstate in this region shares some structural features with the first one, and their difference lies in the dynamical fluctuations of the two components involved in the inter-molecular coordination. When the host-phenyl contacts begin to fall, the phenyl ring of the guest leaves the host center and the dissociation starts. A long low-(free-)energy pathway is observed on the free energy surface. The first structure extracted on this association/dissociation pathway has larger host-guest and host-phenyl contacts and thus features tighter 
host-guest coordination. This binding pose has similar host-guest contacts to the second pose but its hostaromatic contact number is much smaller. Thus, at this position the guest still stays at the host center, but its aromatic ring is more solvent-exposed (i.e., far from the host center). It is worth noting that unlike the GAFF2 case where the partly dissociated structure is of similar thermodynamic stability to the centerbinding pose, under FM-PM6 this host-guest coordination pattern is no longer very stable. When the hostguest and host-phenyl contacts drop further, all components of the inter-molecular coordination become looser. From the last structure extracted on the surface, we know that most parts of the guest have left the host cavity at this position, which serves as an intermediate state along the association/dissociation pathway. As for the protonated G5, the crescent landscape depicting the conformational fluctuations is still observed under FM-PM6, but the free energy surface seems more rugged and the low-(free-)energy region is wider than the GAFF and GAFF2 cases, which suggests a higher level of conformational fluctuations and more dynamical host-guest coordinations. We extracted structures from representative points to understand the detailed differences between the FM-PM6 interaction patterns and the previous results. The first structure locates at the center of the crescent minimum with intermediate values of host-guest and host-phenyl contacts. Although this position on the $C-C_{P h}$ surface often indicates a typical center-binding pose, the flexible FM-PM6 parameter set makes the host-guest coordination different. The twisted host cavity holds the guest molecule, but the shape of the host is quite irregular. One of its $-\mathrm{C}=\mathrm{O}$ portals is close to the $-\mathrm{NH}_{2}{ }^{+}$ group of the guest, but the angle makes it difficult to form hydrogen bonds. The second structure has larger host-guest and host-phenyl contacts than the first one. Compared with the previous center-binding pose, the host-guest coordination at this position is more compact. The host cavity is twisted to fit the guest surface in a better way, which maximizes both the host-guest and the host-phenyl contacts. The last structure has smaller contact numbers and lies at the tail of the crescent minimum. In this binding structure, the phenyl ring of the guest fluctuates outside the host cavity and becomes solvent-exposed. Overall, the basic conformational-fluctuation feature of the CB8-G5 binding observed under the GAFF derivatives remains under FM-PM6, but the host cavity is more twisted than the GAFF derivatives, which is still in agreement with the observations in the previous host-guest complexes (i.e., G1 to G4). The situation of the deprotonated form of G5 is still similar. The basic crescent free energy landscape remains. The structure extracted at the center of the crescent depicts a center-binding pose where the twisted host holds the guest at its central cavity. The other structure locates at the extra horn with larger host-guest and host-phenyl contacts. This pose is similar to that observed in the protonated G5 case, where the distorted host is in a good shape that fits the surface of the guest, thus maximizing the host-guest coordination. As for the next 
structurally complex guest G6, its free energy surface under FM-PM6 is very similar to that under the initial guess GAFF2. A wide free energy minimum is observed for the CB8-G6 complex, but the spread of the FMPM6 basin is narrower than the GAFF2 one. Following the previous GAFF2 analysis, we extract two structures at the same positions as the GAFF2 case, i.e., one at the center of the wide free energy minimum and the other at the small-C tail of the basin. In the first bound structure, the 6-membered rings of the guest except the phenyl moiety are inside the host cavity, while in the second pose, the phenyl and cyclohexyl moieties fluctuate to be solvent-exposed, and only the piperidinium ring penetrates the host cavity. Therefore, it is clear that the basic picture of the host-G6 binding remains unchanged. Namely the distorted host still holds the guest tightly and the guest is fluctuating inside the host cavity. The CB8-G7 surface under FM-PM6 is similar to the GAFF2 one, but only three bound conformations are observed under this parameter set. We can still differentiate them by their host-phenyl contacts. The first one with the largest $C_{P h}$ features the phenyl ring inside the squashed host cavity and the other parts outside (i.e., solventexposed). This binding pose is also observed under the GAFF and GAFF2. However, under GAFF2 there is another pose with slightly smaller host-phenyl contacts in the neighborhood of this binding pose and the barrier between these two states is not high, but this free energy minimum is missing in the current FM-PM6 case. This missing binding pose features a more deeply embedded phenyl ring that comes out from the other side of the host cavity and becomes solvent-exposed and the other parts of the guest closer to the host center, which leads to smaller host-phenyl contacts and larger host-guest contacts. This missing minimum has similar thermodynamic stabilities to the first pose under GAFF2, but is unstable under FM-PM6. Note that under GAFF this missing bound conformation is also of low stability, which agrees with the current FMPM6 case to some extent. Therefore, considering this fact, the host-guest binding under FM-PM6 is somehow sharing some similarities with that produced by the GAFF parameter set. Among the three minima on the $C-C_{P h}$ surface, the second free energy minimum has intermediate values of host-phenyl contacts but the largest host-guest contacts. The structural feature of this binding pose is that only the phenyl moiety is outside the host cavity, while all the other parts of the guest stay at the center of the host ring. Although the FM-PM6 pose is similar to the GAFF2 one, it is worth noting that the host cavity is much more twisted than the GAFF2 case, which agrees with the increased flexibility upon parameter refitting targeting PM6D3H4X. Note that this binding pose is thermodynamically stable under GAFF2 but not under GAFF, which suggests that the host-guest interaction under FM-PM6 shares some similarities with that produced by the GAFF2 parameter set. Among the three conformational states, the last minimum has the smallest host-guest 
and host-phenyl contact numbers, which suggests looser host-guest and host-phenyl coordinations. The basic feature of this binding pose is that the phenyl ring is far away from the host, while the other parts of the guest are relatively close to the center of the squashed host. This binding structure is formed when an intermediate level of association or dissociation is accomplished. Still, compared with the GAFF2 structure, in the FM-PM6 one the host cavity is more twisted and irregularly shaped. Note that this binding pose is thermodynamically very unstable under GAFF and of some stability under GAFF2. Considering this phenomenon, the host-guest coordination produced by FM-PM6 is again similar to GAFF2. Overall, the CB8-G7 binding under FM-PM6 shares similarities with both GAFF and GAFF2. Therefore, considering the behaviors of all host-guest pairs under investigation, the FM-PM6 parameter set produces binding behaviors similar to either GAFF or GAFF2 or both under specific circumstances. However, a worth noting difference is that the FM-PM6 parameters tend to produce a highly flexible host cavity that is often more squashed and twisted than the other cases.

Finally, we check the $C-C_{P h}$ free energy surfaces produced by the FM-BLYP parameter set, as shown in Fig. 8. Under this parameter set, the dynamical behavior of the host is similar to the GAFF2 one, and the guest behaviors are also altered upon GAFF2 refitting. Therefore, the host-guest coordination patterns are expected to be somehow different from the previous GAFF2 ones. This parameter set is also more accurate than the target level of the previous refitted parameter set FM-PM6. Therefore, this FM-BLYP parameter set is considered as the most accurate molecule-specific parameter set employed in the current work and is expected to produce more reliable results than the other. For the structurally simple guest G1, a wide free energy basin is observed in the typical center-binding position, where the guest fluctuates inside the widely open host cavity and the $-\mathrm{NH}_{2}{ }^{+}$group forms hydrogen bonds with $-\mathrm{C}=\mathrm{O}$ portals. The shape of the free energy basin or the distribution of the low-(free-)energy regions and the bound conformations extracted there are all very similar to the GAFF2 results. The situation of the host-guest interactions in the CB8-G2 case is more complex. Under FM-BLYP, the low-(free-)energy regions are significantly different from the previous GAFF, GAFF2 and FM-PM6 cases. The regions with small host-phenyl contacts that are thermodynamically favorable under GAFF2 are relatively unstable under FM-BLYP. The most stable conformational state under this parameter set has the largest $C_{P h}$ among all low-(free-)energy regions. The structural features of the most stable binding pose include that the phenyl moiety connected to the amide bond penetrates the host cavity, while the other parts of the guest stay far away from the host center and are solvent-exposed. The wideness of this large- $C_{P h}$ minimum suggests a significant level of conformational 
fluctuations, where the other solvent-exposed parts of the guest can fluctuate to bind to the surface of the host or become far from any side of the host to minimize the host-guest coordination. The other (meta-)stable binding poses all have smaller host-phenyl contacts, which suggests that the selected phenyl ring is relatively far from the host center in these binding structures. Visualization of the host-guest interactions in these regions suggests many other inter-molecular coordination patterns aside from the phenyl-in-the-cavity one. This phenomenon indicates that the FM-BLYP parameter set has a high level of tolerance of different host-guest interaction patterns and a high level of conformational fluctuations in the bound state, which makes it possible to cover almost all coordination patterns that the host-guest system could form. The CB8-G3 $C-C_{P h}$ surface is as simple as the other cases, where a single free energy minimum featuring the whole guest inside the central cavity and the $-\mathrm{NH}^{+}$and two $-\mathrm{OH}$ groups of the guest forming hydrogen bonds with the $-\mathrm{C}=\mathrm{O}$ portals of the host is observed. Note that the FM-BLYP binding structure is similar to the GAFF and GAFF2 ones instead of the irregularly shaped twisted FM-PM6 one, which is expected considering the host behavior observed previously in unbiased simulations. The guest G4 is structurally similar to G3. The host-guest coordination under GAFF is dominated by the typical centerbinding pose, while under the GAFF2 and FM-PM6 parameter sets many other bound conformations are populated non-negligibly. The refitted FM-BLYP parameter set, instead of being similar to the initial guess GAFF2 and FM-PM6 that is generated in a similar way but targets a lower-level reference, produces dominant center-binding preferences in the CB8-G4 complex. Namely, compared with the CB8-G4 binding patterns under GAFF2 and FM-PM6, the FM-BLYP situation is more reasonable and similar to the CB8-G3 case, where the inter-molecular coordination between the macrocyclic host and the structurally rigid guest is strong/tight and locates at the typical center-binding region. However, despite the structural similarities between the G3 and G4 guests, the G4 free energy surface is still a bit different from the single-minimum G3 one. Specifically, there are three free energy minima on the G4 surface. The distribution of the three minima is similar to the conformational-fluctuation crescent in the CB8-G5 case, which suggests that the FM-BLYP CB8-G4 complex also has some levels of conformational fluctuations. Visualization of bound conformations in the three minima suggests that all of them are center-binding ones, and the differences lie in the degree of distortion to the host cavity and the position of the guest inside the host cavity. The most stable binding pose locates at the middle minimum, where the guest stays at the center of the host and the host cavity is minimally perturbed. Its neighboring small- $C$ state along the $\mathrm{x}$-axis shares most of the binding features of the first minimum, and their host-phenyl contacts are similar. The difference between these two minima mainly lies in the degree of distortion to the host ring. In the small- $C$ minimum, the host 
cavity is distorted and its shape becomes relatively irregular. The other less stable minimum along the y-axis has smaller $C_{P h}$ but larger $C$, which suggests that the host-guest coordination in this case should differ from the other poses in many aspects. The structure extracted here suggests that the host cavity is more squashed than its neighboring state and the phenyl ring of the guest is relatively far from the center of the oval host. The $C-C_{P h}$ surface for the protonated G5 is similar to the GAFF, GAFF2 and FM-PM6 results. The basic conformational-fluctuation picture remains unchanged. Namely, the whole guest stays in the central cavity, the $-\mathrm{NH}_{2}{ }^{+}$group of the guest forms inter-molecular hydrogen bonds with the $-\mathrm{C}=\mathrm{O}$ portals of the host, and the fluctuations of the guest inside the host cavity make some parts of it far away from the host center. As for the deprotonated form of G5, the crescent free energy landscape due to the conformational fluctuations in the bound state is still similar to the results under the other force fields. Namely, the large- $C$ large- $C_{P h}$ position describes a picture that the whole guest penetrates the host cavity, while the other feature parts of the guest fluctuating and leaving the host center. However, compared with the previous results, the free energy basin is quite wide and there is no explicit free energy barrier along the fluctuation pathway, which suggests a high level of conformational fluctuation in the bound state. The CB8-G6 case under FMBLYP differs significantly from the previous GAFF2 and FM-PM6. Instead of the wide free energy minimum featuring significant conformational fluctuations in the bound states observed under the previous cases, under FM-BLYP there are two narrow minima with high free energy barriers between them. This phenomenon suggests that the FM-BLYP CB8-G6 complex has two preferred bound conformations, and the interconversion between them is quite difficult. On the $C-C_{P h}$ surface, the two narrow minima fall in the wide low-(free-)energy basin under GAFF2 and FM-PM6, which suggests only a subset of bound structures during the conformational fluctuations are stable under FM-BLYP. The bound state with larger host-guest and host-phenyl contacts is thermodynamically more favorable than the other. Due to its larger $C$ and $C_{P h}$, it is expected to have more compact host-guest and host-phenyl coordinations. Visualization of the structures there gives us a picture that the cyclohexyl and phenyl moieties of the guest are penetrating the host cavity, while the piperidinium ring is solvent-exposed but with its $-\mathrm{NH}^{+}$group forming one inter-molecular hydrogen bond with one $-\mathrm{C}=\mathrm{O}$ portal of the host. As expected, this binding pose is a snapshot during the conformational fluctuations in the bound state under the initial guess GAFF2 and the FM-PM6 parameter set that is generated in a similar way. The other thermodynamically less favorable conformational state has only the piperidinium moiety penetrating the host cavity, while the other two rings of the guest are far from the 
host center and solvent-exposed. We then turn to the last guest G7. Overall, the CB8-G7 $C-C_{P h}$ surface under FM-BLYP is similar to the GAFF2 and FM-PM6 cases. However, similar to the FM-PM6 case, only a subset of the GAFF2 minima (specifically three out of four) are observed. In the minimum with the largest host-phenyl contacts, the phenyl ring is deeply inserted into the host cavity and even comes out from the other side to be solvent-exposed. The other parts of the guest are close to the host center, which leads to large host-guest contacts. Under GAFF2, conformational fluctuations around this state lead to a two-state behavior in the neighborhood of this binding conformation, while under FM-BLYP only this single fluctuation center remains. As the host behavior under FM-BLYP is very similar to the GAFF2 case, we expect this variation to arise from the changes in the bonded parameters of the guest G7. In the conformational state with intermediate values of host-phenyl contacts, only phenyl leaves the host cavity, while the other parts of the guest stay at the center of the host. The structural features of this bound conformation are extremely similar to the GAFF2 ones. However, this minimum is wider under the refitted parameter set, which suggests that the degree of conformational fluctuations in this state is higher than the GAFF2 case. In the last state on the free energy surface, the whole guest starts to depart from the host cavity. Compared with the previous binding pose, the phenyl ring becomes further away from the host cavity, and the other parts of the guest are also leaving from the host center. As discussed previously, this conformation seems like an intermediate along the binding/unbinding pathway. A worth noting point for the FM-BLYP case is that unlike the GAFF, GAFF2 and FM-PM6 cases where a binding pose is obviously more thermodynamically favorable than the other, these three binding poses are of extremely similar relative free energies (differences similar to the statistical uncertainty) under this more accurate QM-targeted systemspecific parameter set. Thus, these conformational states are populated similarly or have similar weights in the conformational averaging. Overall, the FM-BLYP picture of CB8-drug coordination has its own features and differs from those produced by the other parameter sets in many aspects, but the general picture that the squashed host holds the guest tightly with a significant level of conformational fluctuations in the bound state remains unchanged. Therefore, we can safely conclude that the general picture of the CB8 host-guest binding is indeed caught in our series works.

\subsection{Binding thermodynamics.}

The dynamical behavior of the host ring and the binding-mode investigation shown above depicts the impacts of the refitting of bonded terms. The bonded interactions determine the conformational preference of each molecule. The GAFF and refitted FM-PM6 parameter sets describe a flexible CB8 ring, which can 
easily form a squashed conformation in solvent itself, i.e., in the absence of the external guest. By contrast, the GAFF2 and FM-BLYP parameter sets provide a stiff host cavity that is less energetically favorable in the squashed conformation. However, in the presence of the external guest, the host cavity could still be squashed in order to form tighter host-guest coordination. Due to the difference between the energy costs of distorting the host cavity under different bonded parameter sets and the difference between the bonded terms for the external guest, the strengths of host-guest interactions are altered. First, the binding modes differ under different bonded parameter sets, which would alter the pattern of inter-molecular interactions and thus the strength of host-guest binding. Second, even the host-guest coordination pattern is unchanged, the detailed inter-molecular atom-atom distances are altered, which would also influence the strength of intermolecular electrostatic and vdW interactions and thus the strength of host-guest coordination. Therefore, binding affinities obtained under different bonded parameters are expected to show some differences. Below, we would present a detailed comparison between the predicted binding affinities obtained under different bonded parameter sets and the experimental reference. ${ }^{100}$ Note that the binding free energy is estimated as the sum of the free energy difference between the global minimum and the zero-contact decoupled state and the entropic correction that is used to recover the standard-state definition, as discussed in the computational details section 2.2 and our previous works. ${ }^{83,84}$

The predicted binding thermodynamics and the corresponding statistical uncertainties (standard deviation, SD) under the GAFF derivatives (i.e., GAFF and GAFF2) and the refitted FM-PM6 and FMBLYP parameter sets are summarized in Table 2. The GAFF results are extracted from the best RESP predictions in our previous work, where iterative refitting of the atomic charges is performed to calibrate the charge quality. ${ }^{83}$ Note that these atomic charges are also used in the current newly generated GAFF2, FMPM6 and FM-BLYP datasets. Thus, these predictions only differ in the parameters of the bonded terms. We use three error metrics including the mean signed error (MSE), the MAE, and the RMSE to evaluate the deviations of the predicted binding affinities from the experimental reference, while for the consistency of the predicted and the experimental ranks of binding thermodynamics Kendall's rank correlation coefficient $(\tau)$ and Pearlman's predictive index (PI) are employed. Similar to the previous work, the final estimate of CB8-Ketamine affinity is the average of the protonated and deprotonated results, due to the closeness of the experimental $\mathrm{pKa}(\mathrm{pH} 7.5)$ and the $\mathrm{pH}$ condition that the binding affinities are estimated ( $\mathrm{pH} 7.4)$.

We first check the results obtained with the existing general force field parameters for drug-like molecules, i.e., the GAFF derivatives. Comparing the GAFF and GAFF2 estimates, it is clear that these two GAFF force fields provide different host-guest affinities. For G1, G2, protonated and deprotonated G5, and 
G6, the GAFF2 estimates are higher and also closer to the experimental data than the GAFF results. By contrast, for G3, G4 and G7, the use of GAFF2 parameters leads to smaller binding affinities than the GAFF estimates. For the specific guests G4 and G7, the GAFF2 estimates are significantly worse than the GAFF ones, which is the main source of error in the whole dataset. As a result, although GAFF2 improves the results for most guests, this significant problem results in a bit higher mean errors (e.g., RMSE), which suggests that the GAFF2 is actually of a bit lower performance compared with GAFF. Interestingly, the ranking coefficients are almost unchanged. The correlation between the modelling results and the experimental values is shown in Fig. 9. We can see that although the GAFF2 estimates are closer to the $y=x$ line for 5 systems compared with GAFF, there are 2 guests that are obviously farer from the diagonal than GAFF. There are only two points locating outside the $\pm 2 \mathrm{kcal} / \mathrm{mol}$ line of the experimental results under GAFF, while the number becomes 3 under GAFF2. Namely, there are more 'bad' points under the GAFF2 parameter set. This behavior could, to some extent, help in understanding the increase of RMSE when transferring from GAFF to GAFF2. Overall, considering the small differences between the error and ranking metrics for the GAFF derivatives, their performances on the prediction of binding affinity are similar for the CB8 host-guest systems.

We then turn to the refitted GAFF2 parameter sets. For the lower target level PM6-D3H4X, compared with the initial guess GAFF2, the predicted binding affinities are improved for G1, G2, and protonated G5, remain almost unchanged for G3, and become worse for G4, deprotonated G5, G6 and G7. Overall, the error metrics obtained under the FM-PM6 parameter set are similar to the GAFF2 results. However, obvious differences are observed for the ranking coefficients. The Kendall $\tau$ of FM-PM6 is significantly smaller than GAFF2, while the PI is only marginally lowered. The prediction-experiment correlation for this dataset is also presented in Fig. 9. We can see that for most systems, the GAFF2 and FM-PM6 predictions are similar. The number of 'bad' points with deviations larger than $\pm 2 \mathrm{kcal} / \mathrm{mol}$ from the experimental reference is 3 under this parameter set, and these 3 systems are exactly the outliers in the GAFF2 case. Therefore, the SQM-targeted refitting procedure cannot correct the significant problems of the initial guess GAFF2 in modelling these guests. For the whole dataset, the improvement and decline are of similar magnitudes and the net change is minimal. The above observations suggest that although the refitted force field reproduces the energetics at this computationally efficient semi-empirical level in a better way, it may fail to improve the quality of predictions and produce higher prediction-experiment correlations.

When a higher level BLYP-D4/def2-SVP is selected, we reach the FM-BLYP dataset. This target level is more accurate than the previous PM6-D3H4X, and thus this refitted FM-BLYP parameter set should be 
more accurate the previous FM-PM6. As the FM-BLYP parameters are generated in a system-specific way, they are also considered to be better than the pre-fitted transferable GAFF2 parameters. Checking the statistics in Table 2, we can see that the predicted binding affinities under this parameter set are indeed significantly improved compared with the initial guess GAFF2 and the low-level FM-PM6. Compared with the original GAFF2 results, the predicted binding free energies are improved for all guests except G6. For this specific guest, the original GAFF2 prediction is in perfect agreement with the experimental value, but the deviation of the FM-BLYP result is rather significant. The FM-BLYP results are also significantly better than the FM-PM6 ones, which is expected according to the higher accuracy of the target level. As for the error metrics, the RMSE under this parameter set reaches a very low value of $1.8 \mathrm{kcal} / \mathrm{mol}$, which is much smaller than all other predictions reported so far. The MSE is close to zero, which suggests that there is no systematic overestimation or underestimation observed in the FM-BLYP predictions. The MAE approaches $1.1 \mathrm{kcal} / \mathrm{mol}$, which is also the lowest value observed so far. Significant improvements are also observed for the two ranking coefficients. The correlation of the computed and experimental values under the FM-BLYP parameter set is provided in Fig. 9. The number of 'bad' points falling outside the $\pm 2 \mathrm{kcal} / \mathrm{mol}$ line from the experimental reference is still 2. However, for the other 5 guests, the agreements between the modelling and experimental results are almost perfect. Overall, compared with the other modelling schemes, the combination of RESP charges and FM-BLYP parameters achieves a high-level accuracy, and the predictive power of computational tools in challenging host-guest systems is already in sight.

\subsection{Guidelines for Host-guest Modelling.}

Accurate descriptions of host-guest interactions require accurate inter- and intra-molecular potentials. The inter-molecular interactions are determined by non-bonded electrostatics and vdW terms, with the former (i.e. electrostatics) being more significant. The intra-molecular interactions involve bond stretching, angle bending, and dihedral terms, and the dihedral term plays the most crucial role in determining the conformational preference of each molecule. The interplay between the non-bonded and bonded interactions is the core of molecular recognition. A balanced description of inter- and intra-molecular interactions is needed.

In our previous work focusing on the non-bonded terms, the charge schemes widely used in drug discovery are assessed in great detail and the best-performing regime is identified. ${ }^{83}$ The RESP charge scheme could reproduce the Coulombic molecular ESP very well, leading to accurate descriptions of intermolecular host-guest interactions. However, the conformational dependence of the RESP charge scheme is 
quite significant, while the AM1-BCC charge scheme is more mean-field-like and provides similar results for different charge-generation configurations. Considering this fact, the binding thermodynamics obtained with RESP charges could be inaccurate or wrong when the charge-generation configuration is improperly selected. A solution to this problem recommended in our previous work is using an iterative procedure to parameterize the electrostatics. Specifically, the mean-field-like AM1-BCC model as an initial guess could be used to explore the conformational space, after which the most stable bound conformation is extracted to re-parameterize the molecule with the RESP scheme. Note that the RESP charge scheme could be also used in the initial exploration of the configurational space. Although the RESP scheme is accurate for most systems, it still suffers from the lone-pair problem facing by all fixed-charge models. To further improve the description of electrostatics, polarizable models or even QM calculations should be considered. ${ }^{54,91,132-139}$

In this work, we consider another important influencing factor in MM potentials, the bonded terms. The bonded interactions are not directly involved in the calculation of inter-molecular non-bonded interactions, but still play a crucial role by influencing the intra-molecular conformational preference of each molecule involved in inter-molecular recognitions. The transferable GAFF derivatives for drug-like molecules are assessed, and system-specific parameters are obtained by refitting the bonded terms (bond stretching, angle bending, dihedral) with the generalized FM scheme, which considers both the atomic force, the energy, and various regularization terms to obtain a reasonable set of parameters that reproduce the properties at the target level. The target Hamiltonians/levels determine the ultimate accuracy that the refitted model can achieve. Thus, care should be taken when choosing the target QM level. Here, we selected the dispersion, hydrogen-bonding, and halogen-bonding corrected semi-empirical level PM6-D3H4X and the frequently used and dispersion corrected ab initio level BLYP-D4. Compared with the original GAFF2, the energetics and atomic forces at the target levels are reproduced in a much better way under the refitted parameter sets. However, it should be noted that further improvements could be expected by choosing a higher-level QM target. For instance, with more computational resources, we could use a larger basis set (e.g., def2-TZVP) and choose a more accurate functional such as the double hybrid PWPB95. Further, although the refitted model outperforms the original GAFF2 parameter set in reproducing the energetics at the target QM level, obvious deviations could still be observed. On this aspect, more complicated energy functions (formula) could be used to improve the fitting quality.

With the constructed models to describe the energetics, the solvated host-guest systems could be simulated. Due to the high (free) energy cost of breaking the host-guest coordination and the complex conformational ensemble to explore, some enhanced sampling techniques need to be employed in the 
modelling of host-guest binding. As for the selection of the configurational space to explore, using some $\mathrm{CVs}$ to describe the relative position of the host and the guest seems useful in order to scan their binding patterns. Based on our series of works on host-guest modelling, ${ }^{83,84,97}$ the spherical-coordinates-biased protocol seems sufficient for converged sampling within $\mu$ s-length simulations, although some more CVs should be biased or coupling the simulation with other sampling techniques should be considered if hidden barriers on orthogonal CVs (e.g., internal motions) hinder the convergence of the current protocol.

Overall, for model construction, the combination of the RESP charges and the refitted BLYP-D4 bonded parameters shows the best performance in CB8 host-guest binding. For sampling the configurational space, the spherical-coordinates-biased protocol is sufficient. Thus, we recommend using a similar protocol in the modelling of CB8-related molecules and more generally similar host-guest systems.

\subsection{Further improvements.}

According to our series of works on CB8 host-guest binding simulations, the combination of the RESP charges and some refitted bonded terms provides a balanced accuracy-cost regime for host-guest modelling. The fixed-charge model augmented by an optional iterative refitting procedure provides accurate electrostatics and intra-molecular conformational preferences. If the force-field refitting takes too many computational resources, the GAFF derivatives are also usable. The calculated binding thermodynamics and interaction patterns with the combined model achieve a high level of accuracy within the framework of molecular simulations with classical force fields. The sampling issue is mostly solved by enhanced sampling techniques coupled with a 3D spherical CV set. Although the sampling and Hamiltonian issues are solved mostly, there are still some places that further improvements could be done. Below, we would provide some discussions about several aspects that further developments could be done.

We first consider the sampling of the configurational space. If there are some hidden barriers on orthogonal degrees of freedom, more CVs (e.g., some selected dihedral) could be added to the CV set or coupling other enhanced sampling schemes (e.g., replica exchange) with the current scheme could be considered. Note that this is not really a problem for the current CB8 host-guest systems, which has been discussed and tested in our previous work. ${ }^{83}$ Aside from altering the enhanced sampling regime, modifications on other simulation details (e.g., basic MD parameters) could also be considered. For instance, for the integration of the equations of motion, the widely employed splitting arrangement (i.e., the leapfrog integrator) could be replaced with more efficient schemes such as $\mathrm{BAOAB}^{29,30,140,141}$ to achieve higher accuracy in the configurational space. On this aspect, further improvements could be achieved through 
multiple time scale/step algorithms. ${ }^{31,32,142,143}$

We then consider the description of the system or Hamiltonian, which is actually more problematic than the sampling issue. The Hamiltonian used in the current work is the classical force field, which can be improved by using more accurate forms of potential functions. The electrostatics could be improved by introducing multipoles or fluctuating charges, which provides a better description of the polarization effects that are often claimed to be significant. As this polarization-related issue has been discussed in our previous work, we focus on the forms of bonded terms in the current work. The AMBER derivatives describe the bond stretching and angle bending with harmonic potentials, which could be improved by using more complex functional forms (e.g., cubic or quartic terms) and introducing cross terms to account for the coupling between different motions. It should be noted that the modification of the force-field expression is not limited to the host and the guest molecules. The other components included in the simulation box, i.e., the ions and water molecules, also influence the host-guest coordination and can also be improved. For instance, replacing the simple TIP3P water with more accurate models (e.g., OPC ${ }^{144}$ ) could improve the accuracy of the solvent model, but we should keep in mind that the computational cost is also increased significantly.

Another direction to further improving the Hamiltonian is introducing nuclear quantum effects (NQEs) via the path-integral formulation. The influence of the NQEs on the binding thermodynamics is mostly on hydrogen bonds, the interaction strength of which could alter by about $0.5 \mathrm{kcal} / \mathrm{mol}$. Note that the existence of hydrogen bonds and thus the existence of NQEs are quite general in all host-guest interactions, not just the current CB8 host-guest binding.

\section{Acknowledgement}

This work was supported by the National Natural Science Foundation of China (Grant No. 21633001). Part of the simulation was performed on the high-performance computing platform of the Center for Life Science (Peking University). Dr. Zhaoxi Sun is supported by the PKU-Boya Postdoctoral Fellowship. Dr. Payam Kalhor is supported by the International Postdoctoral Exchange Fellowship (Talent-Introduction Program). We thank the anonymous reviewers for valuable comments and critical reading.

\section{Conflict of Interest Statement}

There are no conflicts of interest to declare. 


\section{Supporting Information Description}

The correlations between the MM and QM energetics at (600 K and) $300 \mathrm{~K}$ for the host and guest molecules for the PM6-D3H4X and BLYP-D4/def2-SVP levels, the time series of force errors $\left(\left\|\Delta \mathbf{F}_{i}\right\|_{2}\right)$ under the original GAFF2 and the refitted parameter sets, dihedral terms defined in GAFF derivatives describing the intra-molecular conformational preference of the host CB8, the time series of the host-guest contacts and the corresponding decomposition into atomic contributions during metadynamics simulations under the GAFF2 and the newly refitted force fields, the 2D radius-contact free energy surfaces obtained under the GAFF2 and the refitted force field are given in the supporting information.

\section{Data Availability}

The data that support the findings of this study are available from the corresponding author upon reasonable request. 


\section{References}

1. Cozzini, P.; Kellogg, G. E.; Spyrakis, F.; Abraham, D. J.; Costantino, G.; Emerson, A.; Fanelli, F.; Gohlke, H.; Kuhn, L. A.; Morris, G. M., Target flexibility: an emerging consideration in drug discovery and design. Journal of medicinal chemistry 2008, 51, 6237-6255.

2. Clark, R. D.; Strizhev, A.; Leonard, J. M.; Blake, J. F.; Matthew, J. B., Consensus scoring for ligand/protein interactions. Journal of Molecular Graphics and Modelling 2002, 20, 281-295.

3. Krammer, A.; Kirchhoff, P. D.; Jiang, X.; Venkatachalam, C.; Waldman, M., LigScore: a novel scoring function for predicting binding affinities. Journal of Molecular Graphics and Modelling 2005, 23, 395-407.

4. Zhang, L.; Li, M.; Liu, Z., A comprehensive ensemble model for comparing the allosteric effect of ordered and disordered proteins. PLoS Comp. Biol. 2018, 14, e1006393.

5. Yu, M.; Chen, Y.; Wang, Z.-L.; Liu, Z., Fluctuation correlations as major determinants of structure-and dynamicsdriven allosteric effects. Phys. Chem. Chem. Phys. 2019, 21, 5200-5214.

6. Mortenson, J. A.; Moriarty, K. M., Ketamine and midazolam anesthesia in Pacific martens (Martes caurina). J. Wildl. Dis. 2015, 51, 250-254.

7. Baker, S. C.; Shabir, S.; Georgopoulos, N. T.; Southgate, J., Ketamine-Induced Apoptosis in Normal Human Urothelial Cells: A Direct, N-Methyl-d-Aspartate Receptor-Independent Pathway Characterized by Mitochondrial Stress. The American journal of pathology 2016, 186, 1267-1277.

8. Balster, R. L.; Woolverton, W. L., Continuous-access phencyclidine self-administration by rhesus monkeys leading to physical dependence. Psychopharmacology 1980, 70, 5-10.

9. McClatchy, D. B.; Savas, J. N.; Martínez-Bartolomé, S.; Park, S. K.; Maher, P.; Powell, S. B.; Yates, J., Global quantitative analysis of phosphorylation underlying phencyclidine signaling and sensorimotor gating in the prefrontal cortex. Mol. Psychiatry 2016, 21, 205-215.

10. Yamamoto, H.; Kamegaya, E.; Sawada, W.; Hasegawa, R.; Yamamoto, T.; Hagino, Y.; Takamatsu, Y.; Imai, K.; Koga, H.; Mishina, M., Involvement of the $\mathrm{N}$-methyl-D-aspartate receptor GluN2D subunit in phencyclidine-induced motor impairment, gene expression, and increased Fos immunoreactivity. Molecular brain 2013, 6, 1-16.

11. Jodo, E., The role of the hippocampo-prefrontal cortex system in phencyclidine-induced psychosis: a model for schizophrenia. Journal of Physiology-Paris 2013, 107, 434-440.

12. Beltran-Campos, V.; Silva-Vera, M.; Garcia-Campos, M.; Diaz-Cintra, S., Effects of morphine on brain plasticity. Neurología (English Edition) 2015, 30, 176-180.

13. Stuart-Harris, R.; Joel, S.; McDonald, P.; Currow, D.; Slevin, M., The pharmacokinetics of morphine and morphine glucuronide metabolites after subcutaneous bolus injection and subcutaneous infusion of morphine. Br. J. Clin. Pharmacol. 2000, 49, 207-214.

14. Shigeev, S., Severity of opiate intoxication to gender and age. Soudni lekarstvi 2007, 52, 21-24.

15. Kim, K.; Selvapalam, N.; Oh, D. H., Cucurbiturils-a new family of host molecules. Journal of inclusion phenomena and macrocyclic chemistry 2004, 50, 31-36.

16. Corma, A.; García, H.; Montes-Navajas, P.; Primo, A.; Calvino, J. J.; Trasobares, S., Gold nanoparticles in organic capsules: a supramolecular assembly of gold nanoparticles and cucurbituril. Chem. Eur. J. 2007, 13, 6359-6364.

17. Wu, X.-L.; Luo, L.; Lei, L.; Liao, G.-H.; Wu, L.-Z.; Tung, C.-H., Highly efficient cucurbit [8] uril-templated intramolecular photocycloaddition of 2-naphthalene-labeled poly (ethylene glycol) in aqueous solution. The Journal of organic chemistry 2008, 73, 491-494.

18. Masson, E.; Ling, X.; Joseph, R.; Kyeremeh-Mensah, L.; Lu, X., Cucurbituril chemistry: a tale of supramolecular success. Rsc Advances 2012, 2, 1213-1247.

19. Saluja, V.; Sekhon, B. S., Calixarenes and cucurbiturils: Pharmaceutial and biomedical applications. Journal of Pharmaceutical Education and Research 2013, 4, 16.

20. Xia, D.; Wang, P.; Ji, X.; Khashab, N. M.; Sessler, J. L.; Huang, F., Functional supramolecular polymeric networks: the marriage of covalent polymers and macrocycle-based host-guest interactions. Chemical Reviews 2020, 120, 6070-6123.

21. Yahiaoui, K.; Seridi, L.; Mansouri, K., Temozolomide binding to Cucurbit[7]uril: QTAIM, NCI-RDG and NBO analyses. $43 / 107$ 
Journal of Inclusion Phenomena and Macrocyclic Chemistry 2021, 99, 61-77.

22. Zhang, X.; Wu, W.; Tao, Z.; Ni, X.-L., Host-guest interactions in nor-seco-cucurbit [10] uril: novel guest-dependent molecular recognition and stereoisomerism. Beilstein journal of organic chemistry 2019, 15, 1705-1711.

23. Sinha, S.; Das Saha, N.; Sasmal, R.; Joshi, D.; Chandrasekhar, S.; Bosco, M. S.; Agasti, S. S., Reversible encapsulations and stimuli-responsive biological delivery from a dynamically assembled cucurbit[7]uril host and nanoparticle guest scaffold. Journal of Materials Chemistry B 2018, 6, 7329-7334.

24. And, S. E. T.; Smithrud, D. B., Carboxylates Stacked over Aromatic Rings Promote Salt Bridge Formation in Water. Journal of the American Chemical Society 2002, 124, 442.

25. Makin, O. S.; Atkins, E.; Sikorski, P.; Johansson, J.; Serpell, L. C., Molecular basis for amyloid fibril formation and stability. Proc. Natl. Acad. Sci. U.S.A. 2005, 102, 315-20.

26. Rani, P.; Biswas, P., Diffusion of hydration water around intrinsically disordered proteins. J. Phys. Chem. B 2015, 119, $13262-13270$.

27. Zerze, G. I. H.; Best, R. B.; Mittal, J., Sequence-and temperature-dependent properties of unfolded and disordered proteins from atomistic simulations. J. Phys. Chem. B 2015, 119, 14622-14630.

28. Sun, Z., A Benchmark Test on the Leapfrog Integrator and its Middle Alternative. 10.26434/chemrxiv.13727839.v1 2021.

29. Leimkuhler, B.; Matthews, C., Robust and efficient configurational molecular sampling via Langevin dynamics. J. Chem. Phys. 2013, 138, 05B601_1.

30. Grønbech-Jensen, N.; Farago, O., A simple and effective Verlet-type algorithm for simulating Langevin dynamics. Molecular Physics 2013, 111, 983-991.

31. Marchi, M.; Procacci, P., Coordinates scaling and multiple time step algorithms for simulation of solvated proteins in the NPT ensemble. J. Chem. Phys. 1998, 109, 5194-5202.

32. Procacci, P.; Marchi, M., Taming the Ewald sum in molecular dynamics simulations of solvated proteins via a multiple time step algorithm. J. Chem. Phys. 1996, 104, 3003-3012.

33. Duan, Y.; Kollman, P. A., Pathways to a protein folding intermediate observed in a 1 -microsecond simulation in aqueous solution. Science 1998, 282, 740-744.

34. Slepoy, A.; Singh, R.; Pazmandi, F.; Kulkarni, R.; Cox, D., Statistical mechanics of prion diseases. Physical review letters 2001, 87, 058101.

35. Shaw, D. E.; Maragakis, P.; Lindorff-Larsen, K.; Piana, S.; Dror, R. O.; Eastwood, M. P.; Bank, J. A.; Jumper, J. M.; Salmon, J. K.; Shan, Y., Atomic-level characterization of the structural dynamics of proteins. Science 2010, 330, 341 -346.

36. Stone, J. E.; Hardy, D. J.; Ufimtsev, I. S.; Schulten, K., GPU-accelerated molecular modeling coming of age. Journal of Molecular Graphics and Modelling 2010, 29, 116-125.

37. Case, D. A.; Cheatham, T. E.; Tom, D.; Holger, G.; Luo, R.; Merz, K. M.; Alexey, O.; Carlos, S.; Bing, W.; Woods, R. J., The Amber Biomolecular Simulation Programs. J. Comput. Chem. 2005, 26, 1668-1688.

38. Abraham, M. J.; Murtola, T.; Schulz, R.; Páll, S.; Smith, J. C.; Hess, B.; Lindahl, E., GROMACS: High performance molecular simulations through multi-level parallelism from laptops to supercomputers. SoftwareX 2015, 1, 19-25. 39. Huai, Z.; Sun, Z., Titration of Adenine in a GA mismatch with Grand Canonical Simulations. Journal of Computational Biophysics and Chemistry 2020, 20, 165-173.

40. Best, R. B.; de Sancho, D.; Mittal, J., Residue-specific $\alpha$-helix propensities from molecular simulation. Biophysical journal 2012, 102, 1462-1467.

41. Makowski, M.; Liwo, A.; Scheraga, H. A., Simple Physics-Based Analytical Formulas for the Potentials of Mean Force of the Interaction of Amino Acid Side Chains in Water. VII. Charged-Hydrophobic/Polar and Polar-Hydrophobic/Polar Side Chains. J. Phys. Chem. B 2017, 121, 379-390.

42. Tobias, D. J.; Brooks III, C. L., Thermodynamics and mechanism of. alpha. helix initiation in alanine and valine peptides. Biochemistry 1991, 30, 6059-6070.

43. Martínezveracoechea, F. J.; Escobedo, F. A., Variance minimization of free energy estimates from optimized expanded ensembles. J. Phys. Chem. B 2008, 112, 8120-8. 
44. Sun, Z.; Yan, Y. N.; Yang, M.; Zhang, J. Z., Interaction Entropy for Protein-Protein Binding. J. Chem. Phys. 2017, 146, 124124.

45. Wang, X.; Sun, Z., A Theoretical Interpretation of Variance-based Convergence Citeria in Perturbation-based Theories. arXiv preprint arXiv:1803.03123 2018.

46. Kästner, J., Umbrella sampling. Wiley Interdisip. Rev. Comput. Mol. Sci. 2011, 1, 932-942.

47. Tiwary, P.; van de Walle, A., Accelerated molecular dynamics through stochastic iterations and collective variable based basin identification. Phys. Rev. B 2013, 87, 094304.

48. Hub, J. S.; De Groot, B. L.; Van Der Spoel, D., g_wham—A Free Weighted Histogram Analysis Implementation Including Robust Error and Autocorrelation Estimates. J. Chem. Theory Comput. 2015, 6, 3713-3720.

49. Kastner, J., Umbrella integration with higher-order correction terms. J. Chem. Phys. 2012, 136, 234102.

50. Wang, X.; Xingzhao, T.; Boming, D.; John Z. H., Z.; Sun, Z., BAR-based Optimum Adaptive Steered MD for Configurational Sampling. J. Comput. Chem. 2019, 40, 1270-1289.

51. Sun, Z.; Gong, Z.; Xia, F.; He, X., Ion Dynamics and Selectivity of Nav channels from Molecular Dynamics Simulation. Chem. Phys. 2021, 111245.

52. Zhu, F.; Hummer, G., Convergence and error estimation in free energy calculations using the weighted histogram analysis method. J. Comput. Chem. 2012, 33, 453-465.

53. Kastner, J., Umbrella integration in two or more reaction coordinates. J. Chem. Phys. 2009, 131, 034109.

54. Sun, Z.; He, Q., Seeding the Multi-dimensional Nonequilibrium Pulling for Hamiltonian Variation: Indirect QM/MM Free Energy Simulations. 10.26434/chemrxiv.13634987.v1 2021.

55. Sun, Z.; Wang, X.; Zhang, J. Z. H.; He, Q., Sulfur-substitution-induced base flipping in the DNA duplex. Phys. Chem Chem. Phys. 2019, 21, 14923-14940.

56. Sun, Z.; Zhang, J. Z. H., Thermodynamic Insights of Base Flipping in TNA Duplex: Force Fields, Salt Concentrations, and Free-Energy Simulation Methods. CCS Chemistry 2021, 3, 1026-1039.

57. Wang, X.; He, Q.; Sun, Z., BAR-Based Multi-Dimensional Nonequilibrium Pulling for Indirect Construction of a QM/MM Free Energy Landscape. Phys. Chem. Chem. Phys. 2019, 21, 6672-6688

58. Sun, Z., BAR-based multi-dimensional nonequilibrium pulling for indirect construction of QM/MM free energy landscapes: from semi-empirical to ab initio. Phys. Chem. Chem. Phys. 2019, 21, 21942-21959

59. Wang, X., Conformational Fluctuations in GTP-Bound K-Ras: A Metadynamics Perspective with Harmonic Linear Discriminant Analysis. J. Chem. Inf. Model. 2021.

60. Sun, Z. X.; Wang, X. H.; Zhang, J. Z. H., BAR-based Optimum Adaptive Sampling Regime for Variance Minimization in Alchemical Transformation. Phys. Chem. Chem. Phys. 2017, 19, 15005-15020.

61. Wang, X.; Tu, X.; Zhang, J. Z. H.; Sun, Z., BAR-based Optimum Adaptive Sampling Regime for Variance Minimization in Alchemical Transformation: The Nonequilibrium Stratification. Phys. Chem. Chem. Phys. 2018, 20, 2009-2021.

62. Munoz, M.; Cardenas, C., How predictive could alchemical derivatives be? Phys. Chem. Chem. Phys. 2017, 19, 1600316012.

63. Wang, B.; Qi, Y.; Gao, Y.; Zhang, J. Z. H., A method for efficient calculation of thermal stability of proteins upon point mutations. Phys. Chem. Chem. Phys. 2020, 22, 8461-8466.

64. Lindahl, V.; Lidmar, J.; Hess, B., Riemann metric approach to optimal sampling of multidimensional free-energy landscapes. Phys. Rev. E2018, 98, 023312.

65. Dewar, M. J. S.; Zoebisch, E. G.; Healy, E. F.; Stewart, J. J. P., Development and use of quantum mechanical molecular models. 76. AM1: a new general purpose quantum mechanical molecular model. Journal of the American Chemical Society 1985, 107, 3902-3909.

66. Elstner, M.; Porezag, D.; Jungnickel, G.; Elsner, J.; Haugk, M.; Frauenheim, T.; Suhai, S.; Seifert, G., Self-consistentcharge density-functional tight-binding method for simulations of complex materials properties. Phys. Rev. B 1998, 58, 7260 .

67. Becke, A. D., Density-functional thermochemistry. IV. A new dynamical correlation functional and implications for exact-exchange mixing. J. Chem. Phys. 1996, 104, 1040-1046. 
68. Hertwig, R. H.; Koch, W., On the parameterization of the local correlation functional. What is Becke-3-LYP? Chem. Phys. Lett. 1997, 268, 345-351.

69. Pople, J. A.; Nesbet, R. K., Self-Consistent Orbitals for Radicals. J. Chem. Phys. 1954, 22, 571-572.

70. Sun, Z.; Zhu, T.; Wang, X.; Mei, Y.; Zhang, J. Z., Optimization of convergence criteria for fragmentation methods. Chem. Phys. Lett. 2017, 687, 163-170.

71. Cieplak, P.; Cornell, W. D.; Bayly, C.; Kollman, P. A., Application of the multimolecule and multiconformational RESP methodology to biopolymers: Charge derivation for DNA, RNA, and proteins. J. Comput. Chem. 1995, 16, 1357-1377.

72. Feig, M.; Onufriev, A.; Lee, M. S.; Im, W.; Case, D. A., Performance comparison of generalized born and Poisson methods in the calculation of electrostatic solvation energies for protein structures. J. Comput. Chem. 2004, 25, 265-84. 73. Hornak, V.; Abel, R.; Okur, A.; Strockbine, B.; Roitberg, A.; Simmerling, C., Comparison of multiple Amber force fields and development of improved protein backbone parameters. Proteins 2006, 65, 712-25.

74. Onufriev, A.; Bashford, D.; Case, D. A., Exploring protein native states and large-scale conformational changes with a modified generalized born model. Proteins Structure Function \& Bioinformatics 2004, 55, 383-94.

75. Sun, Z.; Wang, X., Thermodynamics of Helix formation in small peptides of varying length in vacuo, implicit solvent and explicit solvent: Comparison between AMBER force fields. Journal of Theoretical and Computational Chemistry 2019, 1950015.

76. Desgranges, C.; Delhommelle, J., Towards a machine learned thermodynamics: exploration of free energy landscapes in molecular fluids, biological systems and for gas storage and separation in metal-organic frameworks. Molecular Systems Design \& Engineering 2020.

77. Eken, Y.; Patel, P.; Díaz, T.; Jones, M. R.; Wilson, A. K., SAMPL6 host-guest challenge: binding free energies via a multistep approach. J. Comput.-Aided Mol. Des. 2018, 32, 1097-1115.

78. Peerannawar, S. R.; Rao, S. S.; Gejji, S. P., Density functional investigations on 2-naphthalenecarbonitrile dimerization within cucurbit [8] uril cavitand. J. Mol. Model. 2014, 20, 1-9.

79. Chakraborty, D.; Chattaraj, P. K., Confinement induced thermodynamic and kinetic facilitation of some Diels-Alder reactions inside a CB[7] cavitand. J. Comput. Chem. 2018, 39, 151-160.

80. Ahmadian, N.; Mehrnejad, F.; Amininasab, M., Molecular Insight into the Interaction between Camptothecin and Acyclic Cucurbit[4]urils as Efficient Nanocontainers in Comparison with Cucurbit[7]uril: Molecular Docking and Molecular Dynamics Simulation. J. Chem. Inf. Model. 2020, 60, 1791-1803.

81. Laury, M. L.; Wang, Z.; Gordon, A. S.; Ponder, J. W., Absolute binding free energies for the SAMPL6 cucurbit[8]uril host-guest challenge via the AMOEBA polarizable force field. J. Comput.-Aided Mol. Des. 2018, 32, 1087 -1095.

82. Ali, H. S.; Chakravorty, A.; Kalayan, J.; de Visser, S. P.; Henchman, R. H., Energy-entropy method using multiscale cell correlation to calculate binding free energies in the SAMPL8 host-guest challenge. J. Comput. -Aided Mol. Des. 2021.

83. Sun, Z.; Huai, Z.; He, Q.; Liu, Z., A General Picture of Cucurbit [8] uril Host-Guest Binding. 2021.

84. Sun, Z.; He, Q.; Li, X.; Zhu, Z., SAMPL6 host-guest binding affinities and binding poses from spherical-coordinatesbiased simulations. J. Comput.-Aided Mol. Des. 2020, 34, 589-600.

85. Sun, Z.; Wang, X.; Song, J., Extensive Assessment of Various Computational Methods for Aspartate's pKa Shift. J. Chem. Inf. Model. 2017, 57, 1621-1639.

86. Sun, Z.; Wang, X.; Zhang, J. Z. H., Protonation-dependent Base Flipping in The Catalytic Triad of A Small RNA. Chem. Phys. Lett. 2017, 684, 239-244.

87. Huai, Z.; Yang, H.; Sun, Z., Binding thermodynamics and interaction patterns of human purine nucleoside phosphorylase-inhibitor complexes from extensive free energy calculations. J. Comput.-Aided Mol. Des. 2021.

88. Gordon, J. C.; Myers, J. B.; Folta, T.; Shoja, V.; Heath, L. S.; Onufriev, A., H++: a server for estimating $\mathrm{p} \mathrm{K}$ as and adding missing hydrogens to macromolecules. Nucleic acids research 2005, 33, W368-W371.

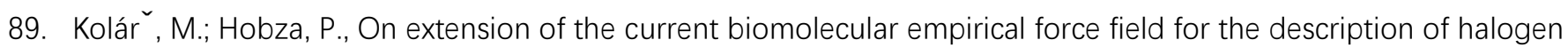
bonds. J. Chem. Theory Comput. 2012, 8, 1325-1333.

90. Li, M.; Zhang, J. Z., Two-bead polarizable water models combined with a two-bead multipole force field (TMFF) for coarse-grained simulation of proteins. Phys. Chem. Chem. Phys. 2017, 19, 7410-7419. 
91. Wang, X.; Yan, J.; Zhang, H.; Xu, Z; Zhang, J. Z., An electrostatic energy-based charge model for molecular dynamics simulation. J. Chem. Phys. 2021, 154, 134107.

92. Zhu, Z.; Wang, G.; Xu, Z.; Chen, Z.; Wang, J.; Shi, J.; Zhu, W., Halogen bonding in differently charged complexes: basic profile, essential interaction terms and intrinsic $\sigma$-hole. Phys. Chem. Chem. Phys. 2019, 21, 15106-15119.

93. Wang, J.; Wolf, R. M.; Caldwell, J. W.; Kollman, P. A.; Case, D. A., Development and testing of a general amber force field. J. Comput. Chem. 2004, 25, 1157-1173.

94. Sun, Z.; Wang, X.; Zhao, Q.; Zhu, T., Understanding Aldose Reductase-Inhibitors interactions with free energy simulation. Journal of Molecular Graphics and Modelling 2019, 91, 10-21.

95. Huai, Z.; Shen, Z.; Sun, Z., Binding Thermodynamics and Interaction Patterns of Inhibitor-Major Urinary Protein-I Binding from Extensive Free-Energy Calculations: Benchmarking AMBER Force Fields. J. Chem. Inf. Model. 2021, 61, 284 297.

96. Procacci, P.; Guarnieri, G., SAMPL7 blind predictions using nonequilibrium alchemical approaches. J. Comput.-Aided Mol. Des. 2021, 35, 37-47.

97. Sun, Z., SAMPL7 TrimerTrip Host-Guest Binding Poses and Binding Affinities from Spherical-Coordinates-Biased Simulations. J. Comput.-Aided Mol. Des. 2021, 35, 105-115.

98. Ercolessi, F.; Adams, J. B., Interatomic potentials from first-principles calculations: the force-matching method. EPL (Europhysics Letters) 1994, 26, 583.

99. Morado, J.; Mortenson, P. N.; Verdonk, M. L.; Ward, R. A.; Essex, J. W.; Skylaris, C.-K., ParaMol: A Package for Automatic Parameterization of Molecular Mechanics Force Fields. J. Chem. Inf. Model. 2021, 61, 2026-2047.

100. Murkli, S.; Klemm, J.; Brockett, A. T.; Shuster, M.; Briken, V.; Roesch, M. R.; Isaacs, L., In Vitro and In Vivo Sequestration of Phencyclidine by Me4Cucurbit [8] uril. Chemistry (Weinheim an der Bergstrasse, Germany) 2020.

101. Bayly, C. I.; Cieplak, P.; Cornell, W.; Kollman, P. A., A well-behaved electrostatic potential based method using charge restraints for deriving atomic charges: the RESP model. J. Phys. Chem. 1992, 97, 10269-10280.

102. Jakalian, A.; Jack, D. B.; Bayly, C. I., Fast, efficient generation of high-quality atomic charges. AM1-BCC model: II. Parameterization and validation. J. Comput. Chem. 2002, 23, 1623-41.

103. Jorgensen, W. L.; Chandrasekhar, J.; Madura, J. D.; Impey, R. W.; Klein, M. L., Comparison of Simple Potential Functions for Simulating Liquid Water. J. Chem. Phys. 1983, 79, 926-935.

104. Price, D. J.; Brooks III, C. L., A Modified TIP3P Water Potential for Simulation with Ewald Summation. J. Chem. Phys. 2004, 121, 10096-10103.

105. Joung, I. S.; Cheatham III, T. E., Determination of Alkali and Halide Monovalent lon Parameters for Use in Explicitly Solvated Biomolecular Simulations. J. Phys. Chem. B 2008, 112, 9020-9041.

106. Joung, I. S.; Cheatham, T. E., Molecular Dynamics Simulations of the Dynamic and Energetic Properties of Alkali and Halide Ions Using Water-Model-Specific Ion Parameters. J. Phys. Chem. B 2009, 113, 13279-13290.

107. Huai, Z.; Yang, H.; Li, X.; Sun, Z., SAMPL7 TrimerTrip host-guest binding affinities from extensive alchemical and endpoint free energy calculations. J. Comput.-Aided Mol. Des. 2021, 35, 117-129.

108. Barducci, A.; Bonomi, M.; Parrinello, M., Metadynamics. Wiley Interdisip. Rev. Comput. Mol. Sci. 2011, 1, 826-843.

109. Valsson, O.; Tiwary, P.; Parrinello, M., Enhancing Important Fluctuations: Rare Events and Metadynamics from a Conceptual Viewpoint. Annual Review of Physical Chemistry 2016, 67, 159.

110. Barducci, A.; Bussi, G.; Parrinello, M., Well-tempered metadynamics: a smoothly converging and tunable free-energy method. Physical Review Letters 2008, 100, 020603.

111. Tiwary, P.; Parrinello, M., A time-independent free energy estimator for metadynamics. J. Phys. Chem. B 2015, 119, 736-42.

112. Tribello, G. A.; Bonomi, M.; Branduardi, D.; Camilloni, C.; Bussi, G., PLUMED 2: New feathers for an old bird. Comput. Phys. Commun. 2014, 185, 604-613.

113. Giovanni, B.; Davide, D.; Michele, P., Canonical sampling through velocity rescaling. J. Chem. Phys. 2007, 126, 2384. 114. Nosé, S.; Klein, M. L., Constant pressure molecular dynamics for molecular systems. Molecular Physics 1983, 50, 1055-1076. 
115. Parrinello, M.; Rahman, A., Polymorphic transitions in single crystals: A new molecular dynamics method. Journal of Applied Physics 1981, 52, 7182-7190.

116. Essmann, U.; Perera, L.; Berkowitz, M. L.; Darden, T.; Lee, H.; Pedersen, L. G., A smooth particle mesh Ewald method. J. Chem. Phys. 1995, 103, 8577-8593.

117. Wang, L.-P.; Chen, J.; Van Voorhis, T., Systematic parametrization of polarizable force fields from quantum chemistry data. J. Chem. Theory Comput. 2013, 9, 452-460.

118. S Brahmkshatriya, P.; Dobes, P.; Fanfrlik, J.; Rezac, J.; Paruch, K.; Bronowska, A.; Lepsik, M.; Hobza, P., Quantum mechanical scoring: structural and energetic insights into cyclin-dependent kinase 2 inhibition by pyrazolo [1, 5-a] pyrimidines. Current computer-aided drug design 2013, 9, 118-129.

119. Becke, A. D., Density-functional exchange-energy approximation with correct asymptotic behavior. Phys. Rev. $A$ 1988, 38, 3098.

120. Lee, C.; Yang, W.; Parr, R. G., Development of the Colle-Salvetti correlation-energy formula into a functional of the electron density. Phys. Rev. B 1988, 37, 785.

121. Weigend, F., Accurate Coulomb-fitting basis sets for H to Rn. Phys. Chem. Chem. Phys. 2006, 8, 1057-1065.

122. Weigend, F.; Ahlrichs, R., Balanced basis sets of split valence, triple zeta valence and quadruple zeta valence quality for $\mathrm{H}$ to Rn: Design and assessment of accuracy. Phys. Chem. Chem. Phys. 2005, 7, 3297-3305.

123. Eichkorn, K.; Weigend, F.; Treutler, O.; Ahlrichs, R., Auxiliary basis sets for main row atoms and transition metals and their use to approximate Coulomb potentials. Theor. Chem. Acc. 1997, 97, 119-124.

124. Eichkorn, K.; Treutler, O.; Öhm, H.; Häser, M.; Ahlrichs, R., Auxiliary basis sets to approximate Coulomb potentials. Chem. Phys. Lett. 1995, 240, 283-290.

125. Caldeweyher, E.; Bannwarth, C.; Grimme, S., Extension of the D3 dispersion coefficient model. J. Chem. Phys. 2017, $147,034112$.

126. Caldeweyher, E.; Ehlert, S.; Hansen, A.; Neugebauer, H.; Spicher, S.; Bannwarth, C.; Grimme, S., A generally applicable atomic-charge dependent London dispersion correction. J. Chem. Phys. 2019, 150, 154122.

127. Stewart, J. J., MOPAC: a semiempirical molecular orbital program. J. Comput.-Aided Mol. Des. 1990, 4, 1-103.

128. Stewart, J. J., MOPAC2016, HTTP://OpenMOPAC.net. Stewart Computational Chemistry, Colorado Springs, CO, USA 2016

129. Neese, F., The ORCA program system. Wiley Interdisip. Rev. Comput. Mol. Sci. 2012, 2, 73-78.

130. Neese, F., Software update: the ORCA program system, version 4.0. Wiley Interdisip. Rev. Comput. Mol. Sci. 2018, 8, e1327.

131. Rocha, G. B.; Freire, R. O.; Simas, A. M.; Stewart, J. J. P., RM1: A reparameterization of AM1 for H, C, N, O, P, S, F, Cl, $\mathrm{Br}$, and I. J. Comput. Chem. 2006, 27, 1101-1111.

132. Bentzien, J.; Muller, R. P.; Florián, J.; Warshel, A., Hybrid ab initio quantum mechanics/molecular mechanics calculations of free energy surfaces for enzymatic reactions: the nucleophilic attack in subtilisin. J. Phys. Chem. B 1998, 102, 2293-2301.

133. Caldararu, O.; Olsson, M. A.; Riplinger, C.; Neese, F.; Ryde, U., Binding free energies in the SAMPL5 octa-acid hostguest challenge calculated with DFT-D3 and CCSD(T). J. Comput.-Aided Mol. Des. 2016, 1-20.

134. Olsson, M. A.; Söderhjelm, P.; Ryde, U., Converging ligand-binding free energies obtained with free-energy perturbations at the quantum mechanical level. J. Comput. Chem. 2016, 37, 1589-1600.

135. Jackson, N. E.; Webb, M. A.; de Pablo, J. J., Layered nested Markov chain Monte Carlo. J. Chem. Phys. 2018, 149, 072326.

136. Wang, X.; Liu, J.; Zhang, J. Z.; He, X., Electrostatically embedded generalized molecular fractionation with conjugate caps method for full quantum mechanical calculation of protein energy. J. Phys. Chem. A 2013, 117, 7149-7161.

137. Kitaura, K.; Ikeo, E.; Asada, T.; Nakano, T.; Uebayasi, M., Fragment molecular orbital method: an approximate computational method for large molecules. Chem. Phys. Lett. 1999, 313, 701-706.

138. Sun, Z.; Liu, Z., BAR-Based Multi-Dimensional Nonequilibrium Pulling for Indirect Construction of QM/MM Free Energy Landscapes: Varying the QM Region. Adv. Theory Simul. 2021, 2100185. 
139. Song, J.; Ji, C.; Zhang, J. Z., The critical effect of polarization on the dynamical structure of guanine quadruplex DNA. Phys. Chem. Chem. Phys. 2013, 15, 3846-3854.

140. Leimkuhler, B.; Matthews, C., Rational construction of stochastic numerical methods for molecular sampling. Applied Mathematics Research eXpress 2012, 2013, 34-56.

141. Zhang, Z.; Liu, X.; Chen, Z.; Zheng, H.; Yan, K.; Liu, J., A unified thermostat scheme for efficient configurational sampling for classical/quantum canonical ensembles via molecular dynamics. J. Chem. Phys. 2017, 147, 034109.

142. Procacci, P.; Berne, B., Computer simulation of solid C60 using multiple time-step algorithms. J. Chem. Phys. 1994, 101, 2421-2431.

143. Bou-Rabee, N., Time integrators for molecular dynamics. Entropy 2014, 16, 138-162.

144. Izadi, S.; Anandakrishnan, R.; Onufriev, A. V., Building Water Models, A Different Approach. J. Phys. Chem. Lett. 2014, 5, 3863-3871. 
Table 1. The names of the CB8 host and 7 drugs/guests, the experimental binding affinities in $\mathrm{kcal} / \mathrm{mol}$, the pKa values of all guests determined experimentally at $298 \mathrm{~K}$ and via ChemAxon, and the net charges considered in the current work. The net charge of each molecule is determined by the relative magnitude of its $\mathrm{pKa}$ and the experimental condition ( $\mathrm{pH} 7.4)$ that the binding thermodynamics are measured. The experimental and ChemAxon predicted values of all guests except G5 (Ketamine) are obviously larger than the experimental condition $\mathrm{pH}$ 7.4. Thus, all of them are protonated at the nitrogen atom in our modelling. As for the guest G5, both the experimental and the ChemAxon results indicate the existence of acid-base equilibria. Thus, both the protonated and deprotonated forms are considered.

\begin{tabular}{cccccc}
\hline Molecule & Name & $\Delta G_{\text {exp }}$ & $p K_{a, \exp }$ & $p K_{a, \text { ChemAxon }}$ & Net Charge Considered \\
\hline CB8 & Cucurbit[8]uril & - & - & - & 0 \\
G1 & Methamphetamine & -7.05 & 9.87 & 10.21 & 1 \\
G2 & Fentanyl & -9.93 & 8.99 & 8.77 & 1 \\
G3 & Morphine & -11.63 & 8.21 & 9.12 & 1 \\
G4 & Hydromorphone & -11.22 & 8.2 & 8.59 & 1 \\
G5 & Ketamine & -12.32 & 7.5 & 7.45 & 0 or 1 \\
G6 & PhenylCyclohexylPiperidine & -14.07 & 8.29 & 10.56 & 1 \\
G7 & Cocaine & -7.92 & 8.61 & 8.85 & 1 \\
\hline
\end{tabular}


Table 2. The CB8-guest binding affinities in kcal/mol. $\Delta G_{\text {exp }}$ is the experimental value. $\Delta G_{\mathrm{GAFF}}$ and $\Delta G_{\mathrm{GAF} 2}$ denote the binding affinity obtained with the existing general force fields of GAFF and GAFF2, respectively, while $\Delta G_{\mathrm{FM}-\mathrm{PM} 6}$ and $\Delta G_{\mathrm{FM}-\mathrm{BLYP}}$ represent the binding free energy obtained with our newly fitted bonded parameter sets targeting PM6-D3H4X and BLYP-D4/def2-SVP, respectively. SD represents the standard error of the free energy estimate, which is obtained from block analysis. MSE, MAE, RMSE, $\tau$, and PI serve as quality measurements. Due to the closeness of the pKa of G5 Ketamine and the pH condition that the experimental binding affinities are measured, the protonated and deprotonated forms of G5 are considered to be approximately equally populated. As a result, the final estimate of CB8-G5 binding affinity is the average of the protonated and deprotonated G5 results, which is used to calculate the quality metrics, while protonation-dependent results are presented for a detailed comparison of the simulated results.

\begin{tabular}{|c|c|c|c|c|c|c|c|c|c|c|}
\hline Host & Guest & $\Delta G_{\text {exp }}$ & $\Delta G_{\mathrm{GAFF}}$ & SD & $\Delta G_{\mathrm{GAFF} 2}$ & SD & $\Delta G_{\mathrm{FM}-\mathrm{PM} 6}$ & SD & $\Delta G_{\mathrm{FM}-\mathrm{BLYP}}$ & SD \\
\hline \multirow{8}{*}{ CB8 } & G1 & -7.05 & -5.9 & 0.4 & -8.0 & 0.4 & -7.4 & 0.4 & -7.4 & 0.4 \\
\hline & $\mathrm{G} 2$ & -9.93 & -8.2 & 0.5 & -11.3 & 0.5 & -9.1 & 0.5 & -10.0 & 0.5 \\
\hline & G3 & -11.63 & -14.9 & 0.4 & -8.5 & 0.5 & -8.6 & 0.5 & -11.4 & 0.4 \\
\hline & G4 & -11.22 & -9.9 & 0.5 & -5.5 & 0.5 & -6.6 & 0.4 & -14.7 & 0.4 \\
\hline & G5 prot & -12.32 & -3.5 & 0.5 & -8.3 & 0.5 & -6.4 & 0.5 & -9.2 & 0.5 \\
\hline & G5 deprot & -12.32 & -10.4 & 0.5 & -10.9 & 0.5 & -8.0 & 0.5 & -16.0 & 0.5 \\
\hline & G6 & -14.07 & -12.4 & 0.5 & -14.1 & 0.5 & -12.8 & 0.5 & -11.0 & 0.4 \\
\hline & G7 & -7.92 & -8.1 & 0.5 & -6.9 & 0.4 & -9.2 & 0.5 & -8.2 & 0.4 \\
\hline RMSE & & & 2.6 & & 2.8 & & 2.9 & & 1.8 & \\
\hline MSE & & & -1.1 & & -1.5 & & -1.9 & & 0.2 & \\
\hline MAE & & & 2.1 & & 2.1 & & 2.4 & & 1.1 & \\
\hline$\tau$ & & & 0.4 & & 0.4 & & 0.0 & & 0.5 & \\
\hline PI & & & 0.6 & & 0.6 & & 0.5 & & 0.7 & \\
\hline
\end{tabular}




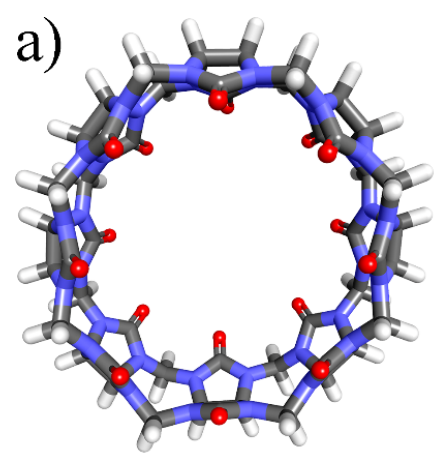

Cucurbit[8]uril<smiles>CN[C@@H](C)Cc1ccccc1</smiles>

G1 Methamphetamine

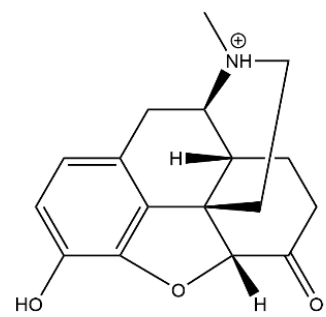

G4 Hydromorphone CyclohexylPiperidine<smiles>CCC(=O)N(c1ccccc1)C1CCN(CCc2ccccc2)CC1</smiles>

G2 Fentanyl<smiles>CN[C@@]1(c2ccccc2Cl)CCCCC1=O</smiles>

G5 Ketamine protonated

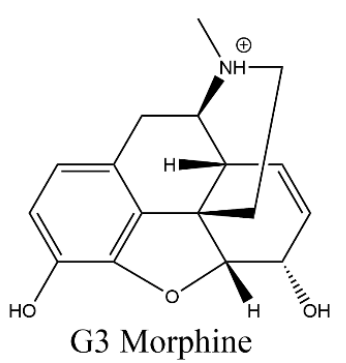

G3 Morphine

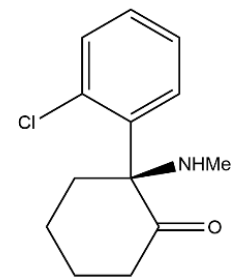

G5 Ketamine deprotonated

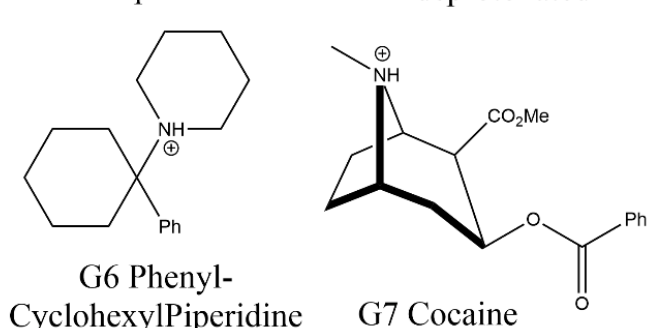

b)

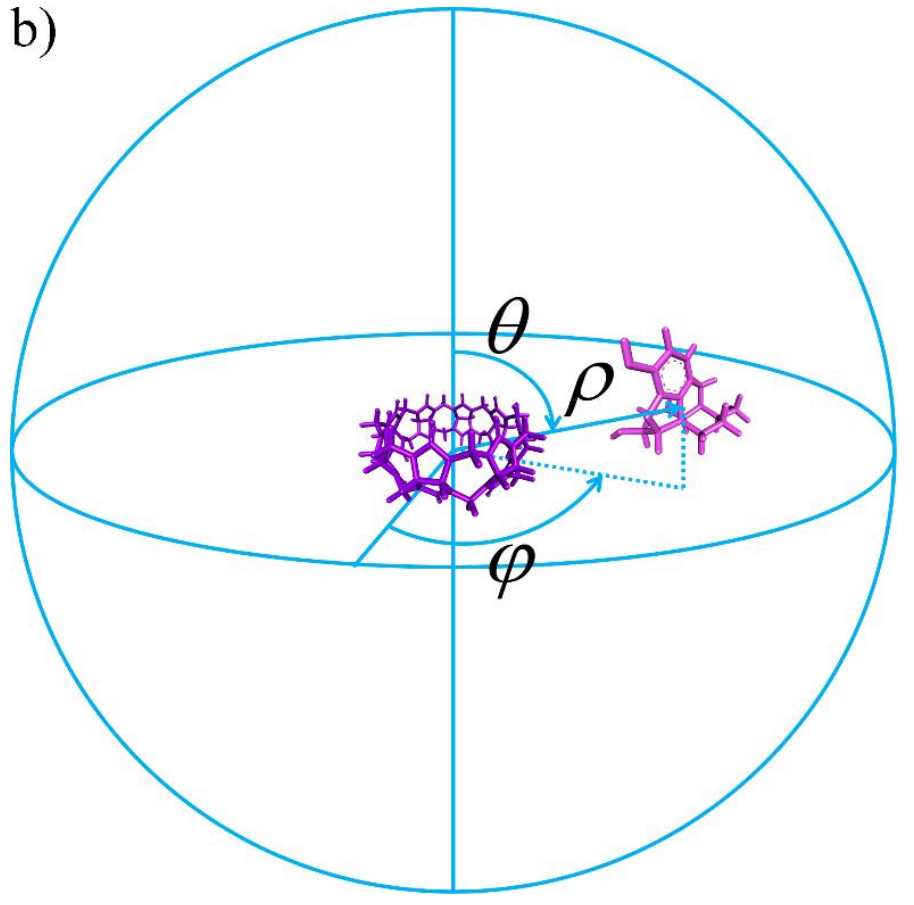

Fig. 1. a) The 3D structure of the CB8 host and the 2D chemical structures of its 7 guests. For the guest G5 Ketamine, due to the small difference between the experimental and ChemAxon predicted $\mathrm{pKa}$ and the $\mathrm{pH}$ condition that the binding affinity is measured, both the protonated and deprotonated forms of the guest G5 are considered in our modelling. b) An illustration of the 3D spherical coordinates CV used to bias the simulation. 


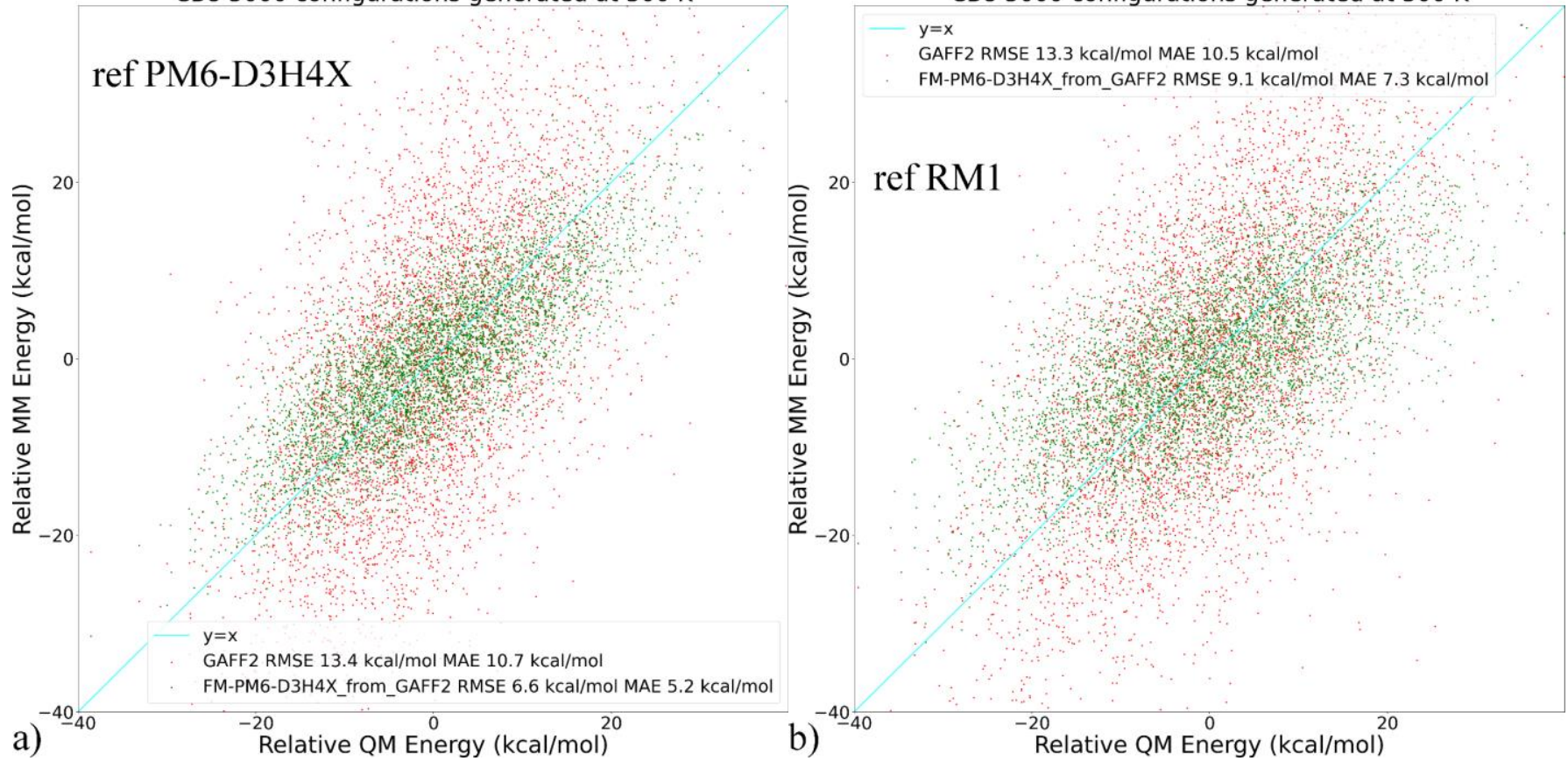

$>50 \mathrm{kcal} /(\mathrm{mol} \cdot A)>30 \mathrm{kcal} /(\mathrm{mol} \cdot A)>10 \mathrm{kcal} /(\mathrm{mol} \cdot \AA)$ CB8 GAFF2 Force by-atom error 5000 Configs at $300 \mathrm{~K}$ RMSE $40.05 \mathrm{kcal} /\left(\mathrm{mol} * \mathrm{~A}^{*}\right.$ atom)

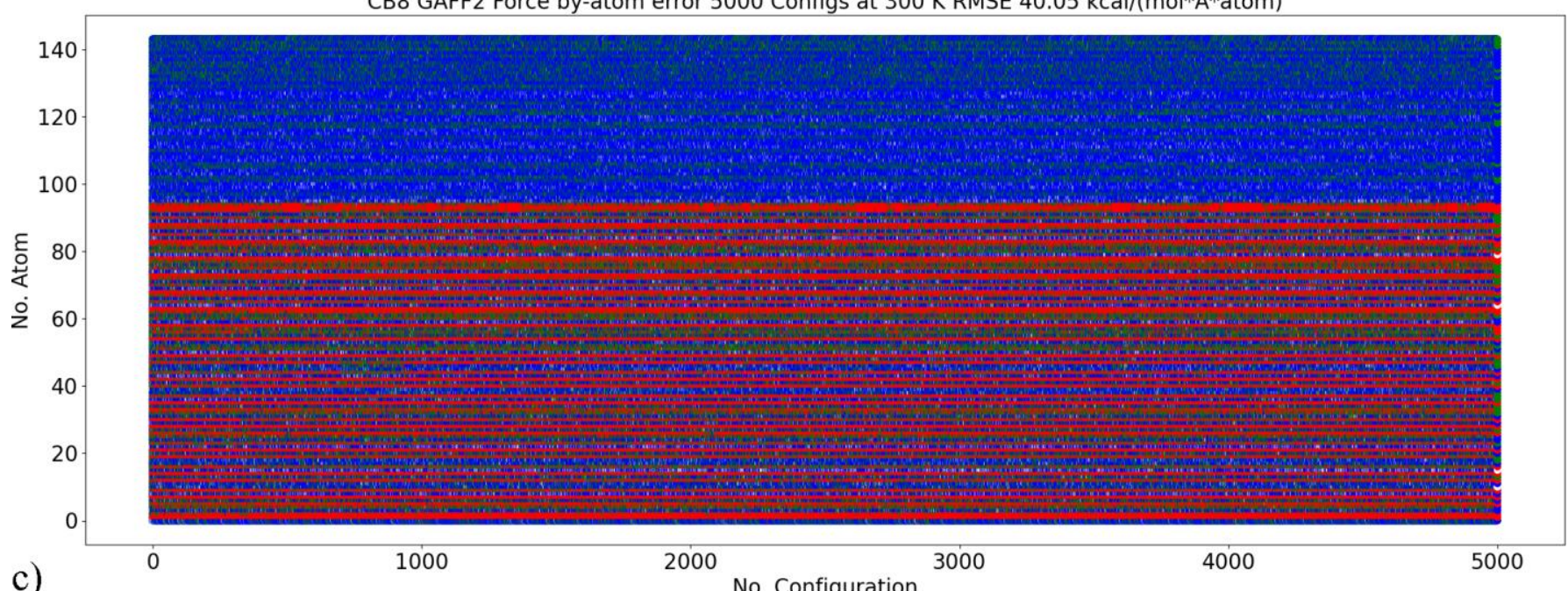

c)

CB8 FM-PM6-D3H4X_from_GAFF2 Force by-atom error 5000 Configs at $300 \mathrm{~K}$ RMSE $17.87 \mathrm{kcal} /\left(\mathrm{mol} * \mathrm{~A}^{*}\right.$ atom)

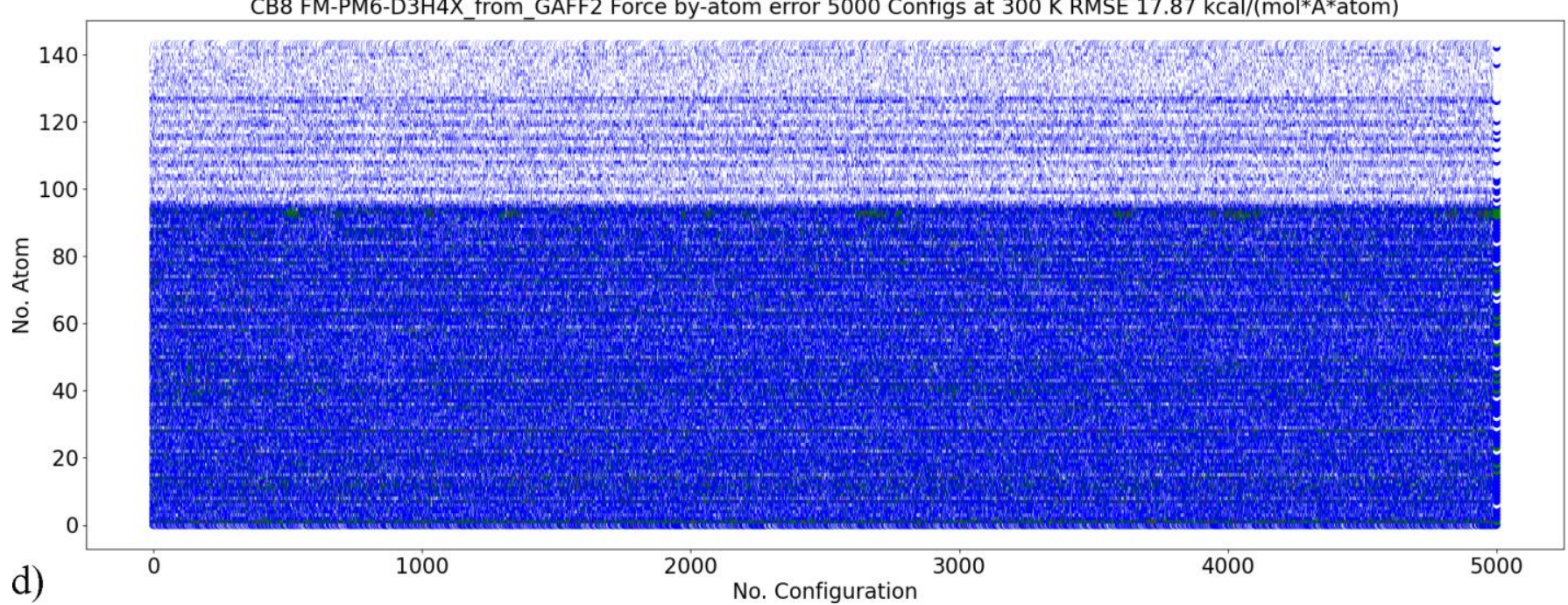



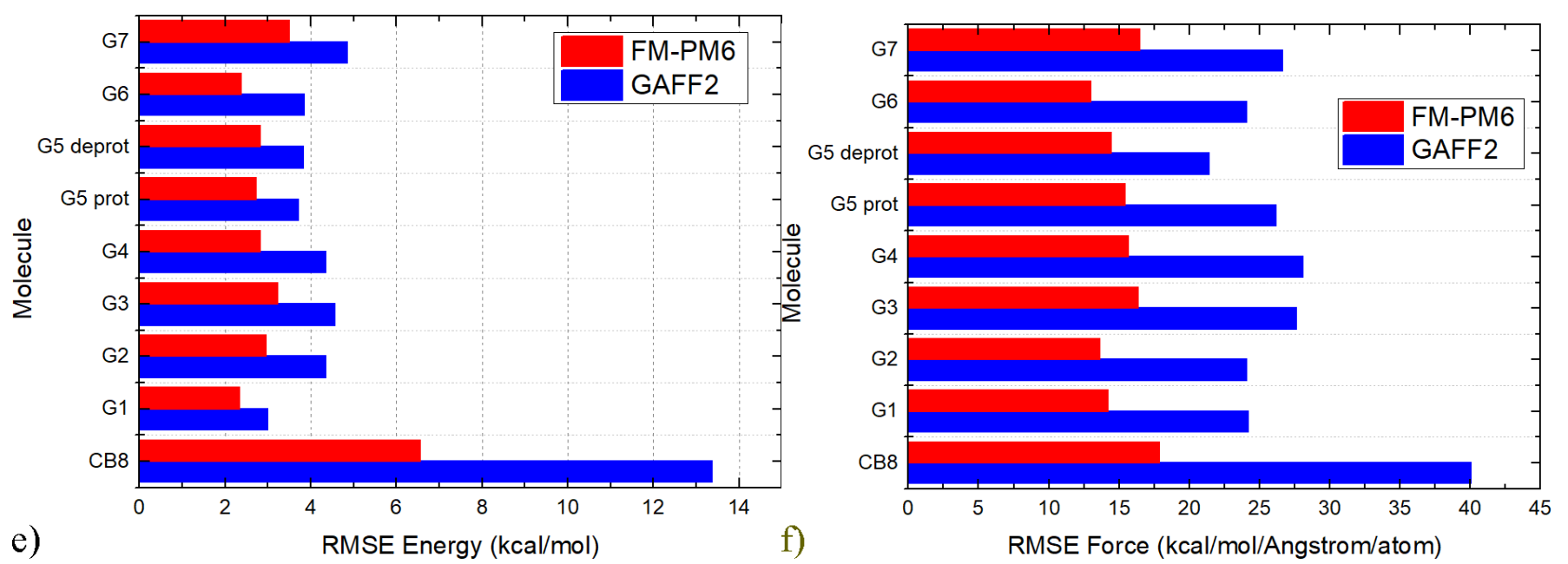

Fig. 2. a) The correlations between the MM (i.e., GAFF2 and FM-PM6) and PM6-D3H4X energetics calculated from $25 \mathrm{~ns}$ trajectories generated at $300 \mathrm{~K}$ in vacuo for the host, b) those between energetics produced by MM force fields and a different SQM Hamiltonian of RM1 instead of the target PM6-D3H4X. c-d) The time series of errors of atomic forces (i.e., Frobenius norm of $\Delta \mathbf{F}$ ) produced by GAFF2 and the refitted FM-PM6 force field calculated with configurations generated at $300 \mathrm{~K}$ for the host CB8. Red dots for the force errors larger than $50 \mathrm{kcal} /(\mathrm{mol} \cdot \AA)$, green for force errors larger than $30 \mathrm{kcal} /(\mathrm{mol} \cdot \AA)$, blue for errors larger than $10 \mathrm{kcal} /(\mathrm{mol} \cdot \AA)$, and white for the other small-error points. The first 96 atoms are nonhydrogen heavy atoms, the force errors of which are larger than the other hydrogen atoms. The errors/deviations (RMSEs) of e) the energetics and f) atomic forces produced by GAFF2 and the refitted FM-PM6 force field calculated with configurations generated at $300 \mathrm{~K}$ for all host and guest molecules under investigation. 


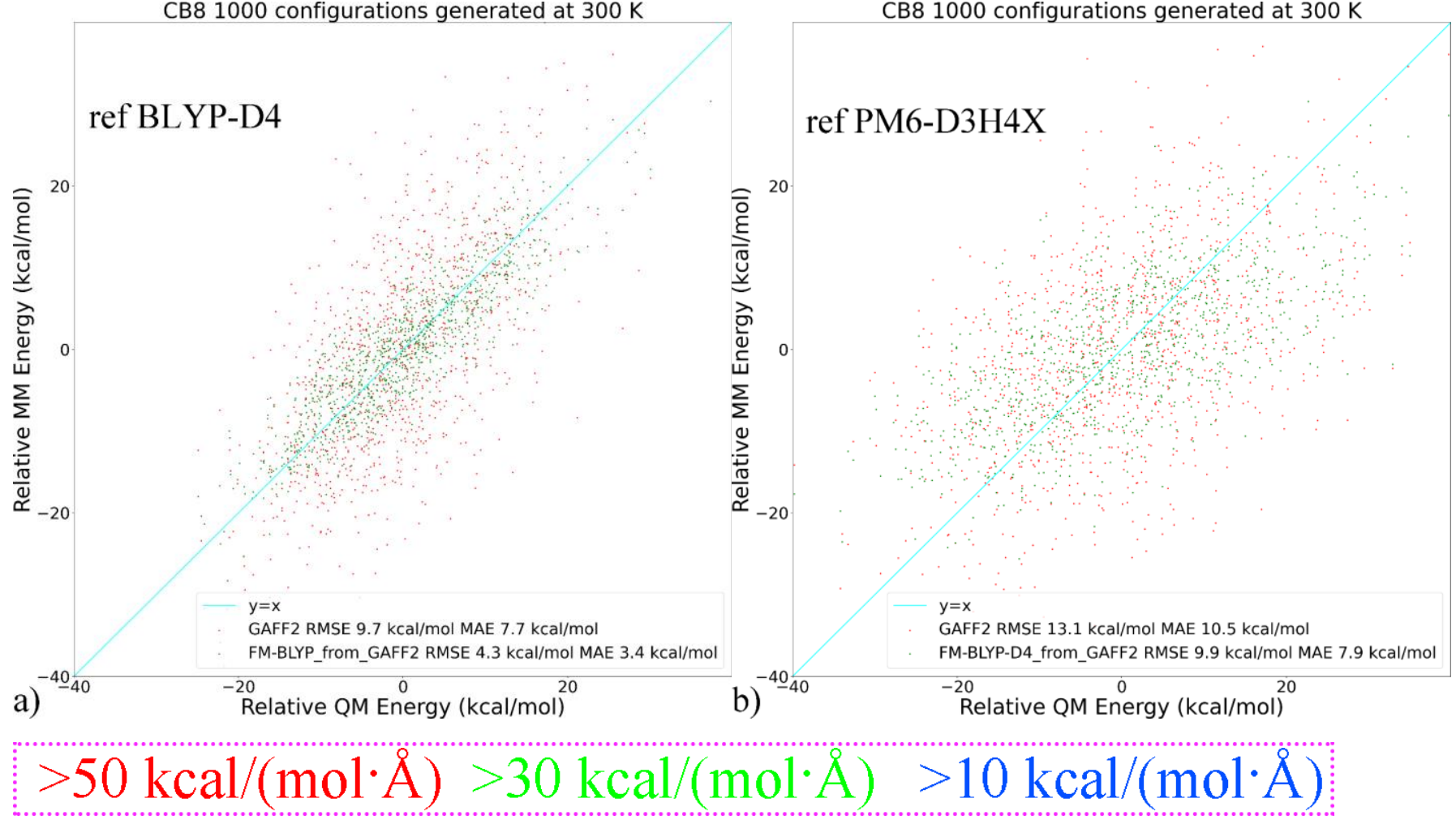

CB8 GAFF2 Force by-atom error 1000 Configs at $300 \mathrm{~K} \mathrm{RMSE} 30.06 \mathrm{kcal} /\left(\mathrm{mol} * A^{*}\right.$ atom)

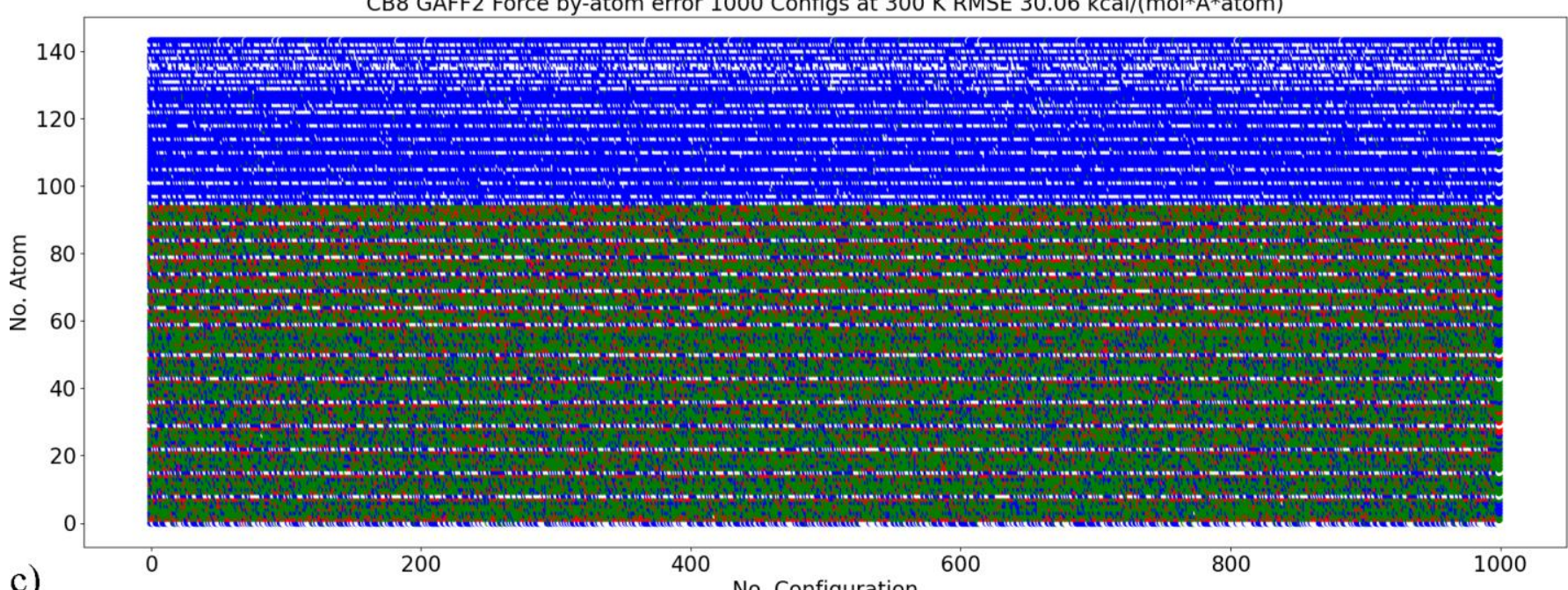

c)

No. Configuration

CB8 FM-BLYP-D4_from_GAFF2 Force by-atom error 1000 Configs at $300 \mathrm{~K}$ RMSE $13.07 \mathrm{kcal} /\left(\mathrm{mol} * \mathrm{~A}^{*}\right.$ atom)

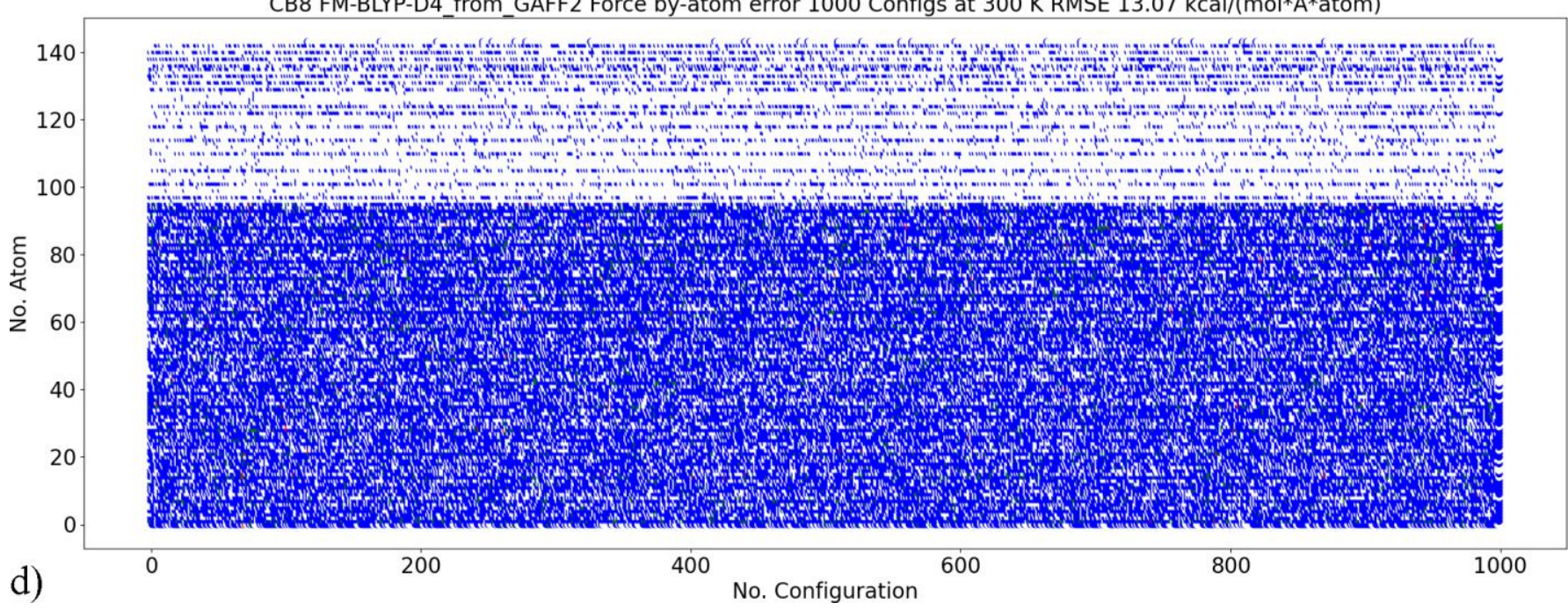



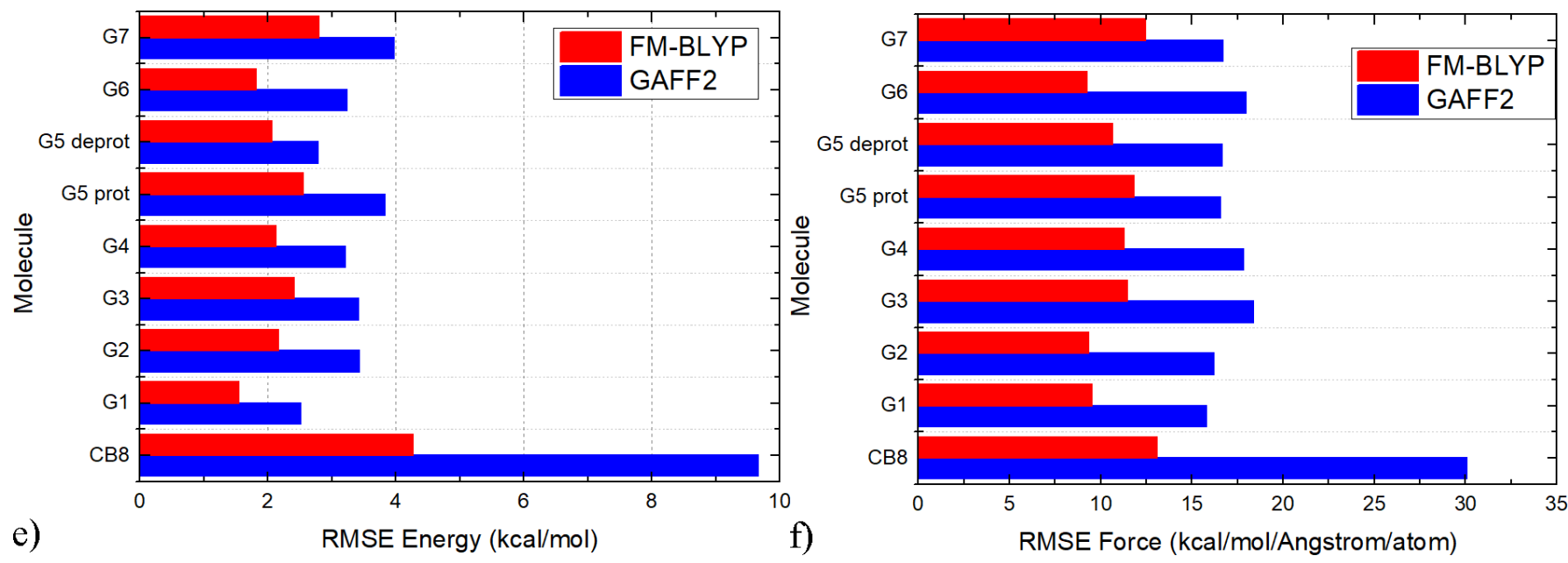

Fig. 3. a) The correlations between the MM (i.e., GAFF2 and FM-BLYP) and BLYP-D4 energetics calculated from $10 \mathrm{~ns}$ trajectories generated at $300 \mathrm{~K}$ in vacuo for the host, b) those between the energetics produced by MM and a different reference level of PM6-D3H4X (i.e., the previous target in FM-PM6 refitting) instead of the target level BLYP-D4. Note that for this reference level, the FM-BLYP parameter set produces larger errors compared with the previous FM-PM6, which is fitted directly to the data at this reference level. c-d) The time series of errors of atomic forces produced by GAFF2 and the refitted FMBLYP force field calculated with configurations generated at $300 \mathrm{~K}$ for the host CB8. Red dots for the force errors larger than $50 \mathrm{kcal} /(\mathrm{mol} \cdot \AA)$, green for force errors larger than $30 \mathrm{kcal} /(\mathrm{mol} \cdot \AA)$, blue for errors larger than $10 \mathrm{kcal} /(\mathrm{mol} \cdot \AA)$, and white for the other small-error points. The first 96 atoms are non-hydrogen heavy atoms, the force errors of which are larger than the other hydrogen atoms. The errors/deviations of e) energetics and f) atomic forces produced by GAFF2 and the refitted FM-BLYP force field calculated with configurations generated at $300 \mathrm{~K}$ for all host and guest molecules under investigation. 

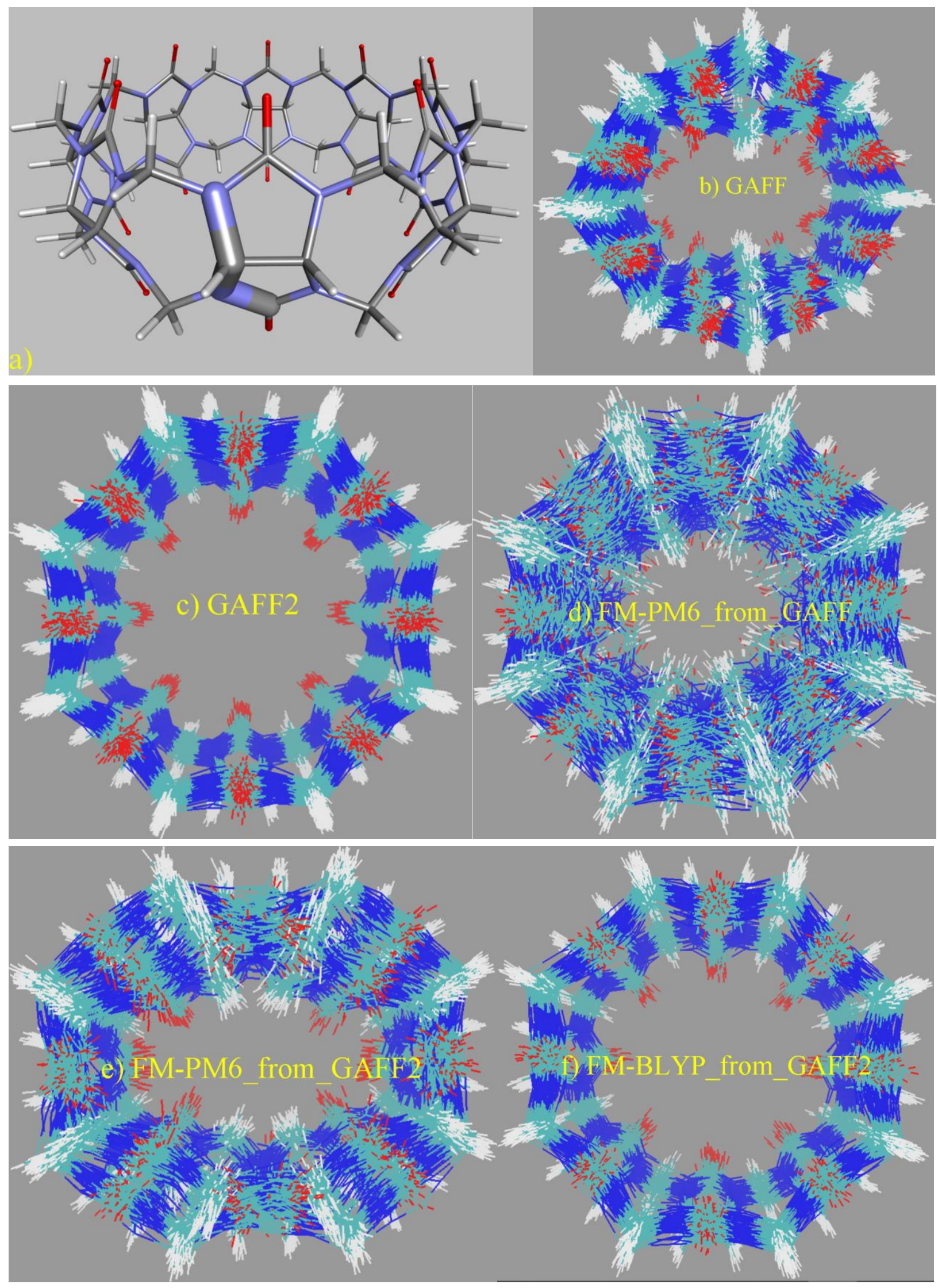


\section{$\mathrm{Rg}$ of host}

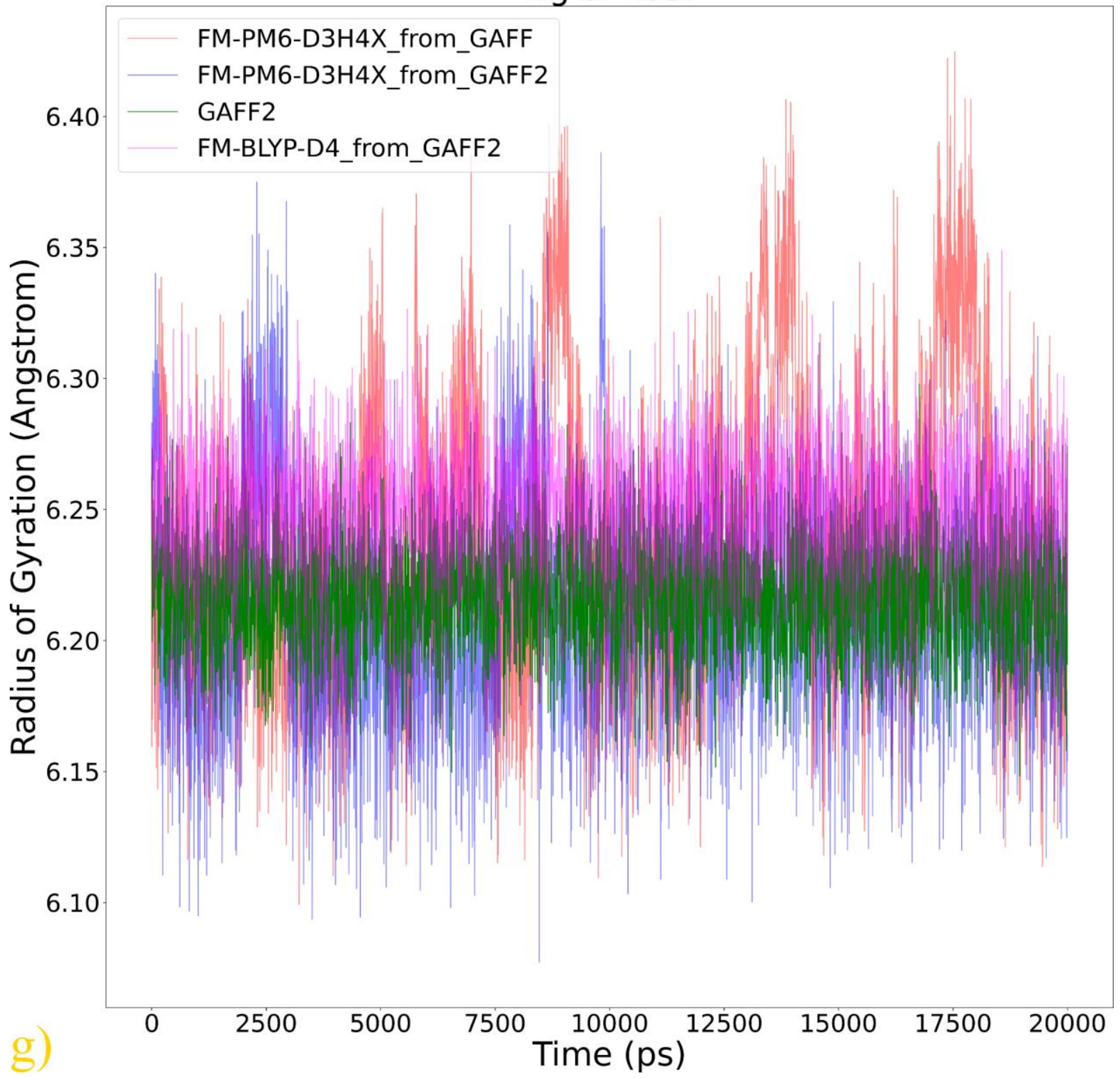

Fig. 4. a) The dihedral term that shows the most significant difference between GAFF derivatives describing the conformational preference (stiffness) of the host. The superposition of the host configurations during 20 ns unbiased simulations in explicit solvent with bonded parameters from b) GAFF, c) GAFF2, d) the FMPM6 parameters initiated from GAFF, e) the FM-PM6 parameters initiated from GAFF2, and f) FM-BLYP initiated from GAFF2. g) The time series of the radius of gyration of the CB8 ring under the GAFF2 and the refitted parameter sets. For the target PM6-D3H4X, two parameter sets are fitted initiated from GAFF and GAFF2 parameters, respectively, while for the target BLYP-D4 the initial guess used is only GAFF2. 

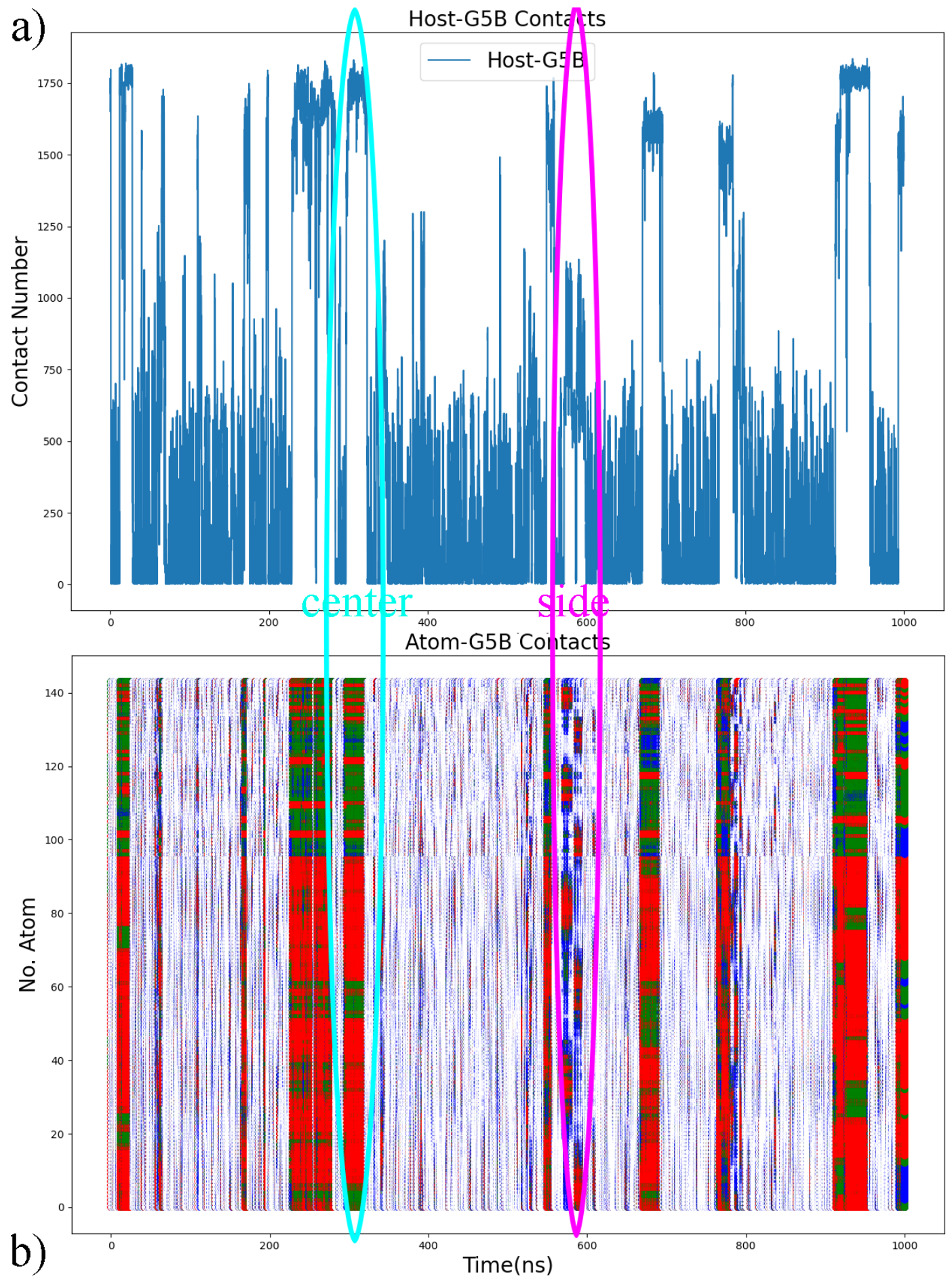

Fig. 5. a) The number of contacts between all atoms of the host CB8 and the guest deprotonated G5 and b) its decomposition by each atom of the host during 1000 ns enhanced sampling simulations with the newly refitted FM-PM6 parameters. The y-axis represents the serial number of host atom. The first 96 atoms of the host are heavy atoms, and the other are hydrogen atoms. As all atoms of the host and the guest are included in the calculation, the number of contacts differ from the later projection of the free energy landscapes. Red dots denote contacts larger than 10, green dots represent contact number between 5 and 10, blue ones are those larger than 1, and the other are represented by white dots. The cyan oval provides an example of the typical center-binding pose explored during enhanced sampling simulations, while in the magenta one there 
is a side-binding mode. The results of all host-guest complexes under the GAFF2 and the refitted FM-PM6 and FM-BLYP parameter sets are provided in Fig. S6-S8. 

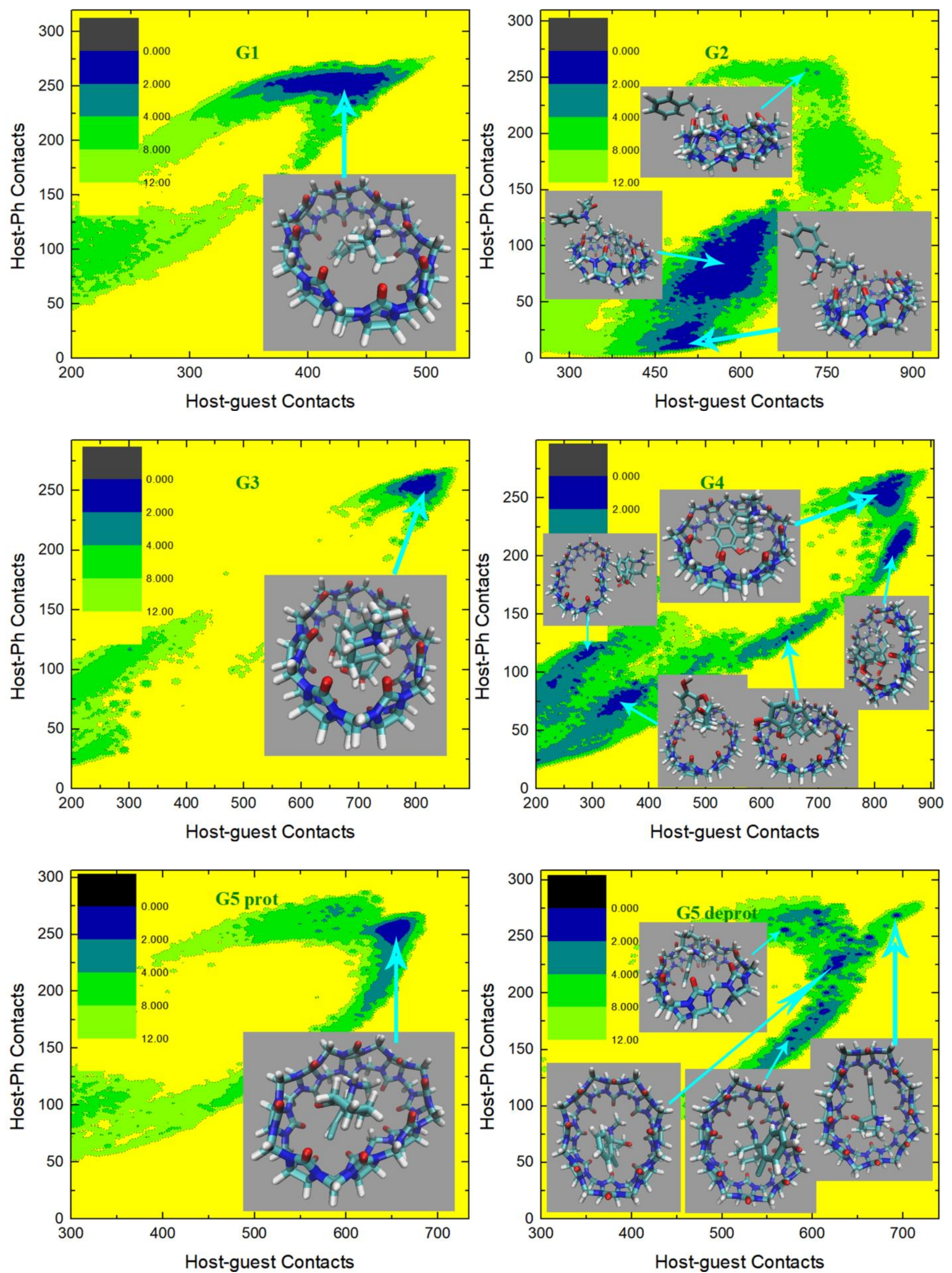

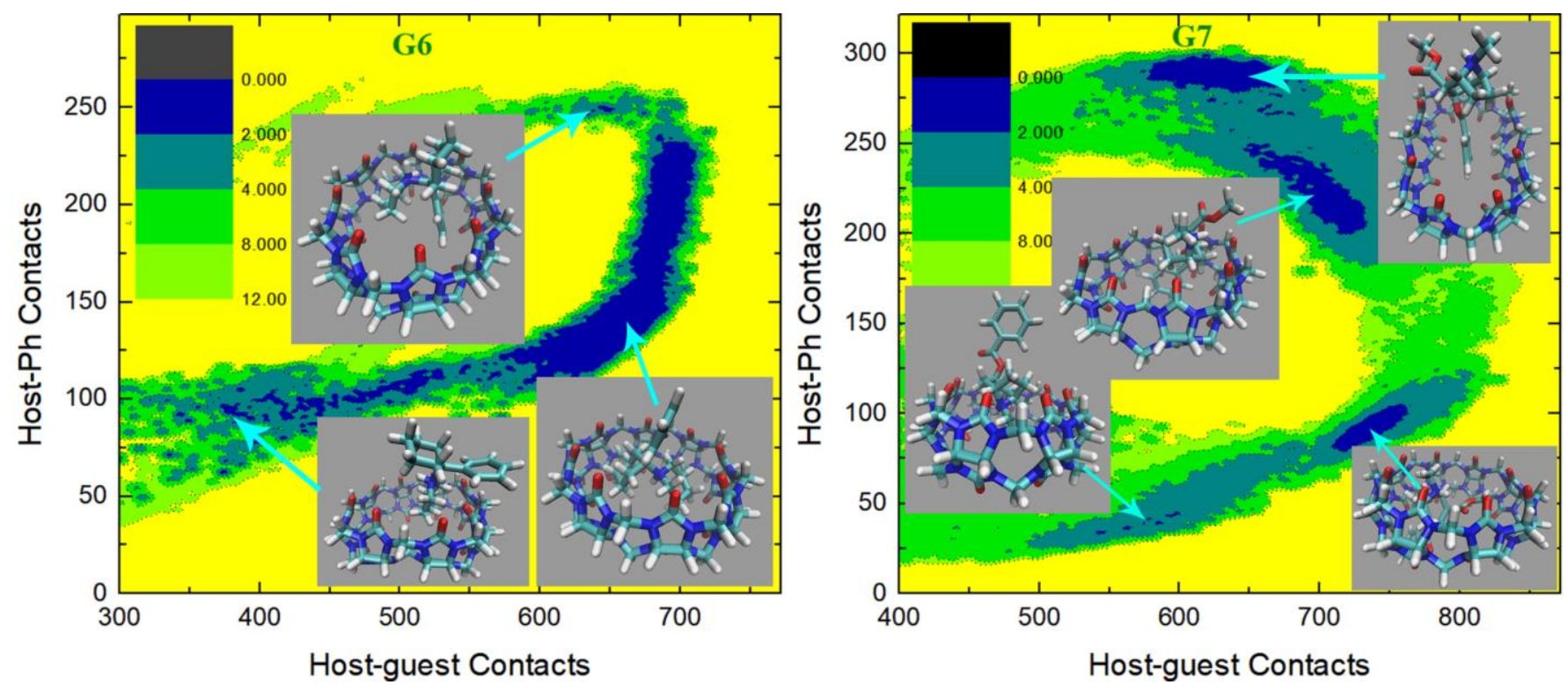

Fig. 6. $2 \mathrm{D} C-C_{P h}$ free energy surfaces in $\mathrm{kcal} / \mathrm{mol}$ obtained under the GAFF 2 force field. 

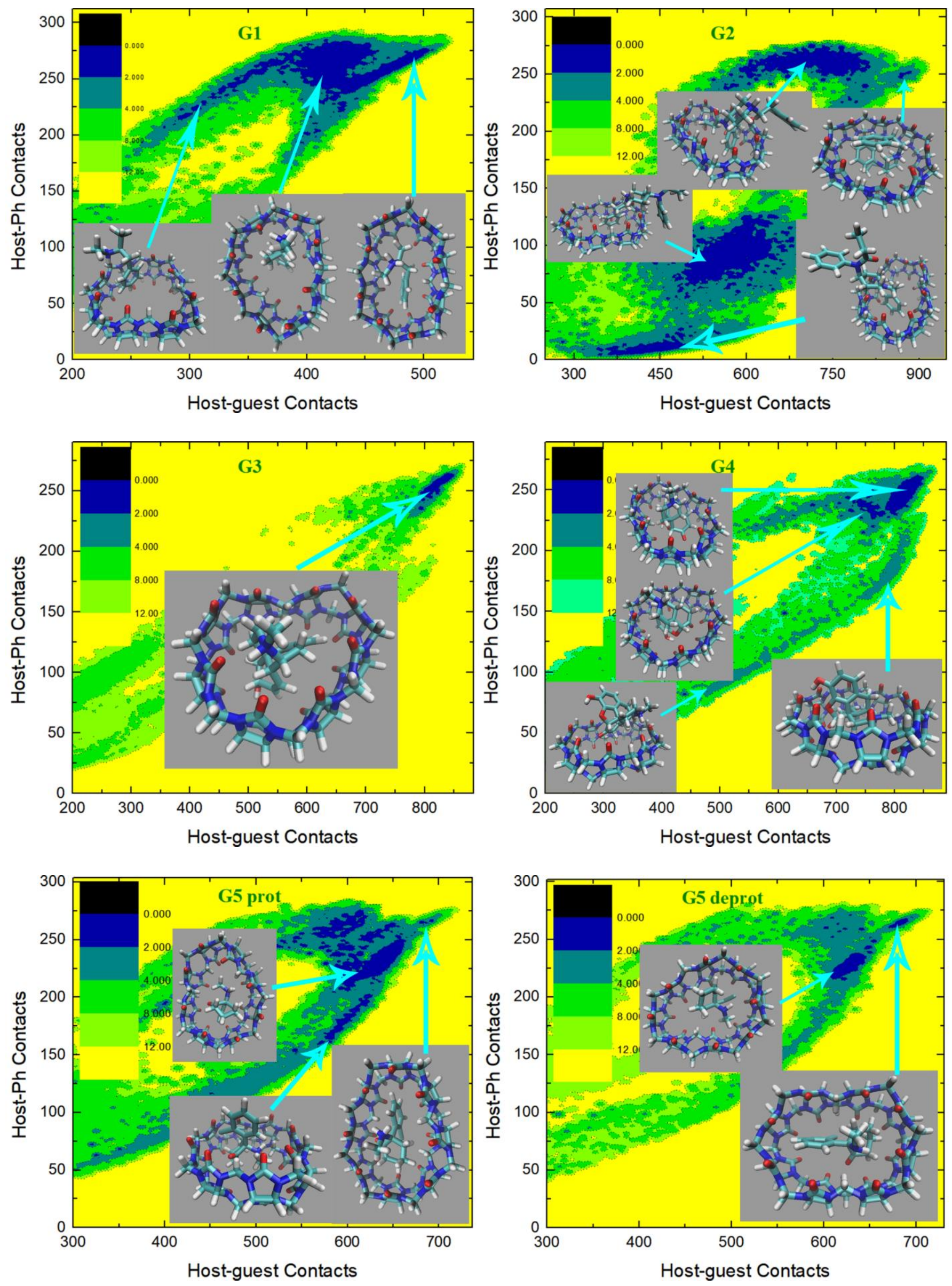

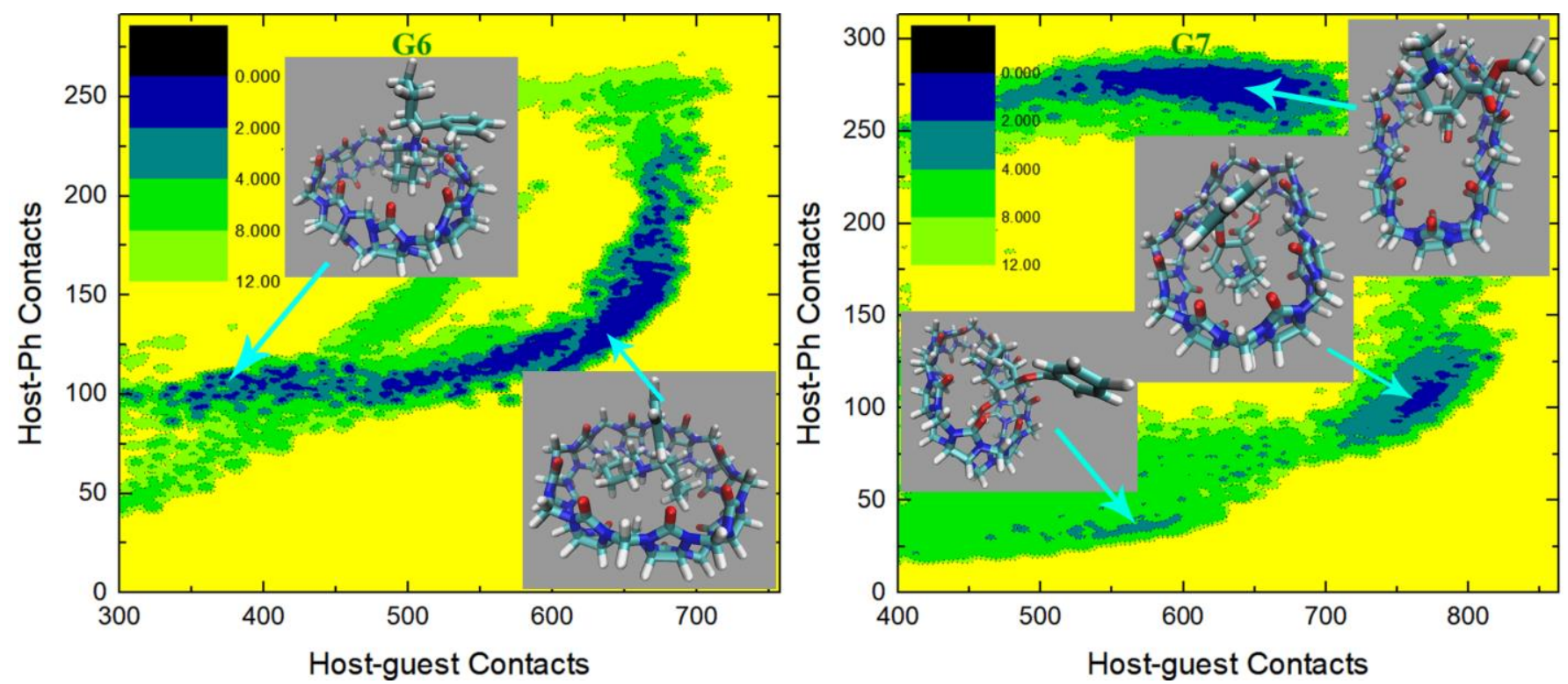

Fig. 7. 2D $C-C_{P h}$ free energy surfaces in $\mathrm{kcal} / \mathrm{mol}$ obtained under the newly refitted force field FM-PM6. 

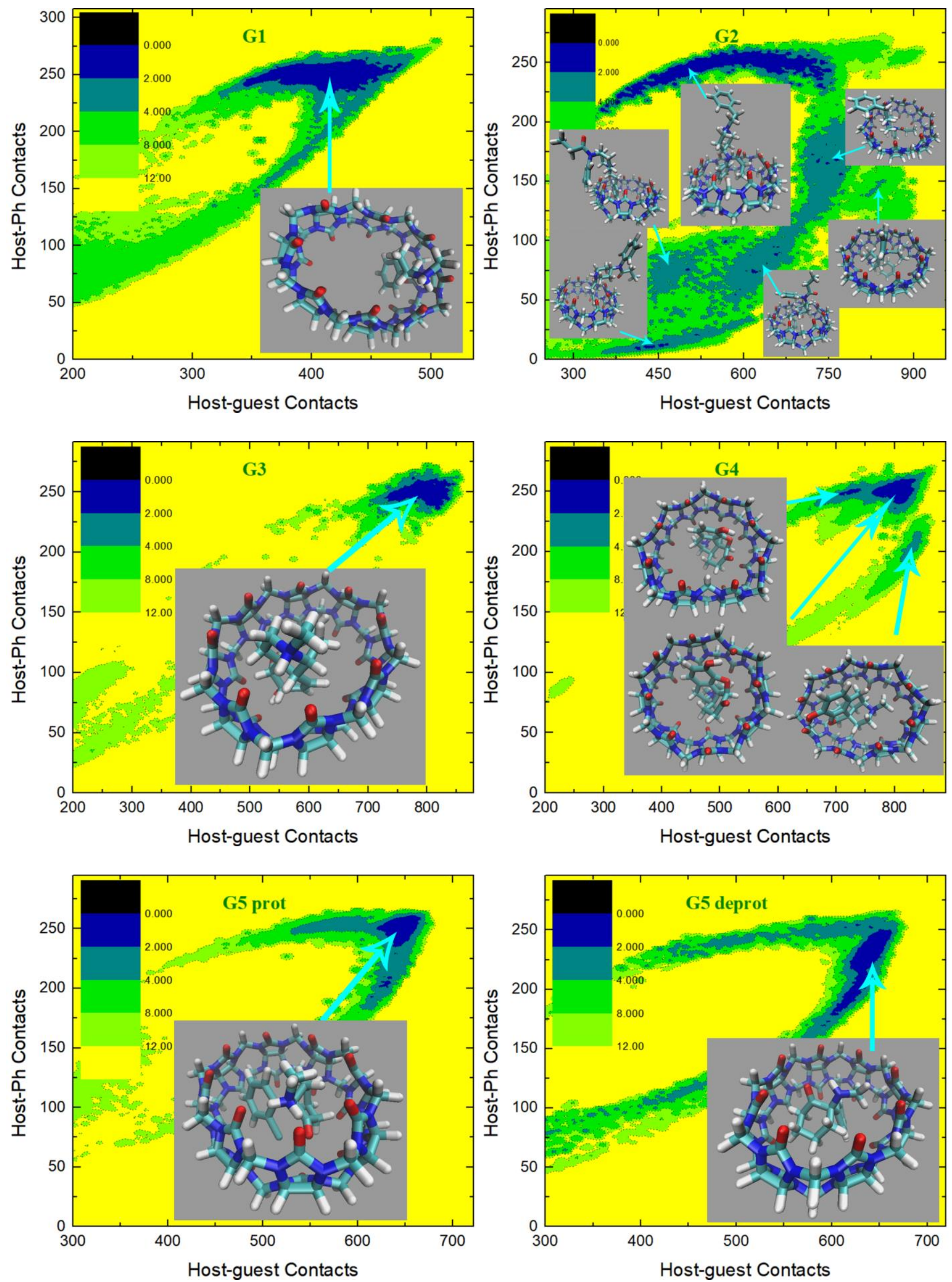

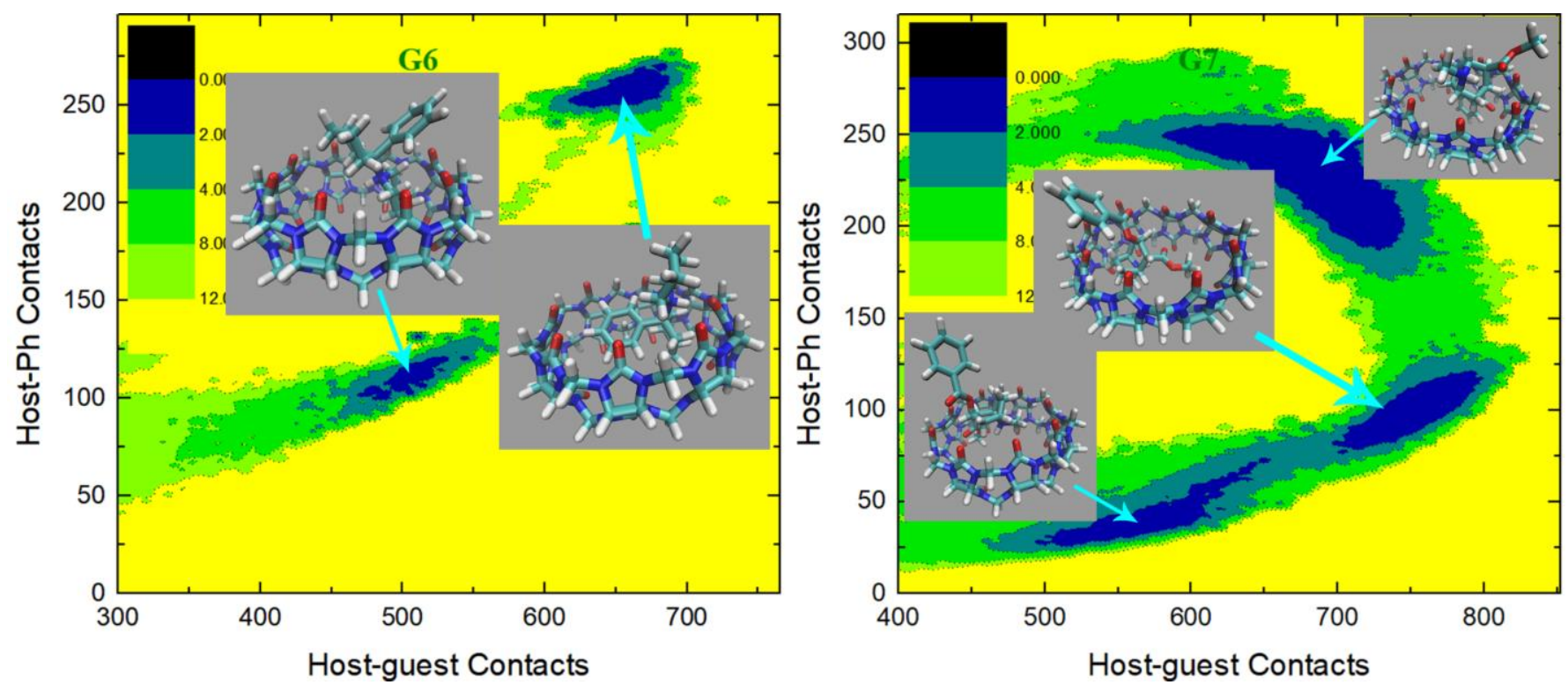

Fig. 8. 2D $C-C_{P h}$ free energy surfaces in $\mathrm{kcal} / \mathrm{mol}$ obtained under the newly refitted force field FMBLYP. 


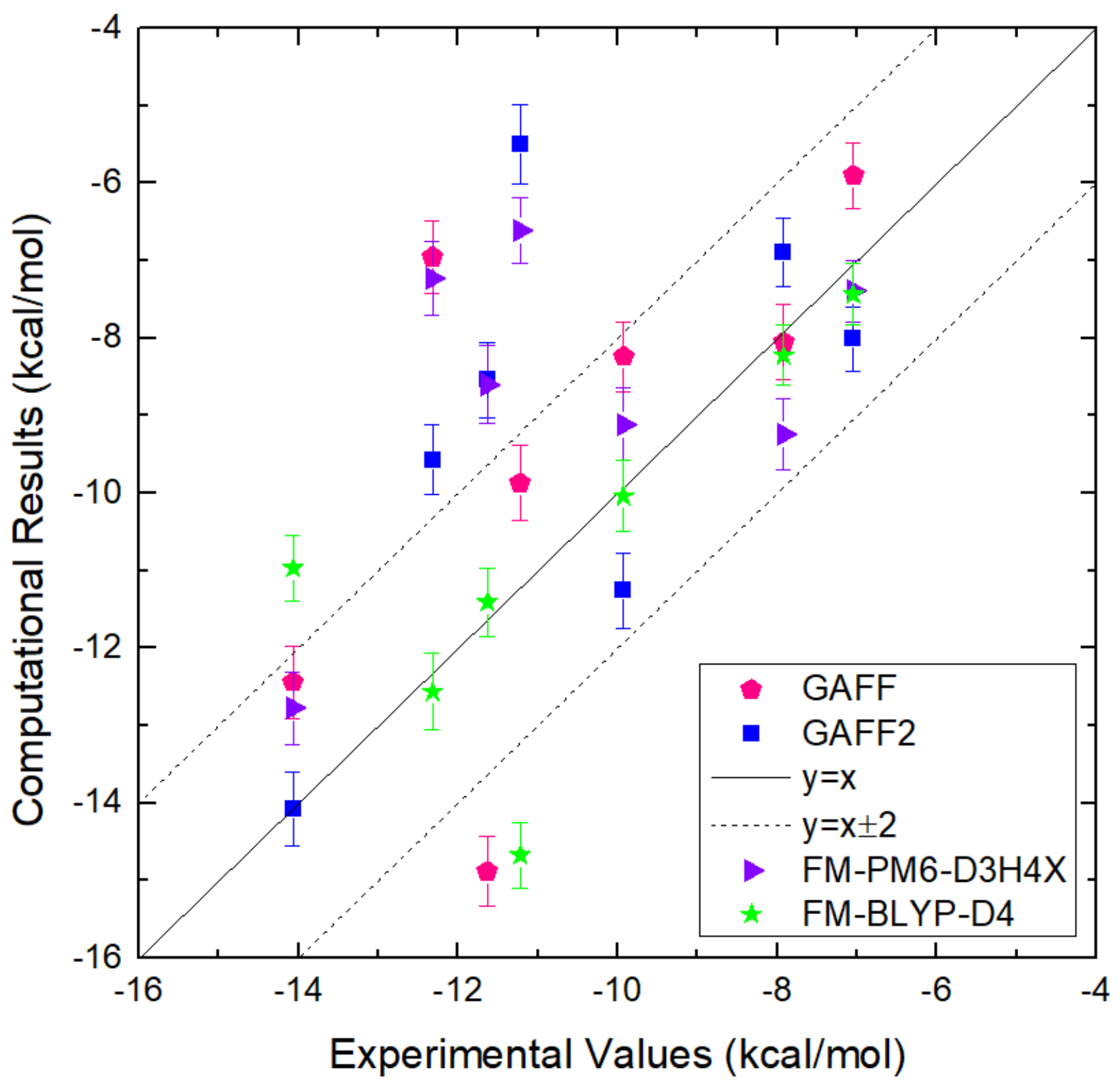

Fig. 9. Correlation between the binding affinities obtained from our computational modeling and the experimental reference for CB8-guest systems. The exact values of the binding affinities are presented in Table 2. 


\section{Supporting Information: A General Picture of Cucurbit[8] uril Host-Guest Binding: Recalibrating Bonded Interactions}

Zhaoxi Sun ${ }^{1}$, Qiaole $\mathrm{He}^{2}$, Zhihao Gong ${ }^{1}$, Zhe Huai ${ }^{3}$, Payam Kalhor ${ }^{1}$, Zhirong Liu ${ }^{1}$

${ }^{I}$ Beijing National Laboratory for Molecular Sciences, Institute of Theoretical and Computational Chemistry, College of Chemistry and Molecular Engineering, Peking University, Beijing 100871, China

${ }^{2}$ Enzymaster (Ningbo) Bio-Engineering Co., Ltd., North Century Avenue 333, 315100 Ningbo, China

${ }^{3} X t a l P i$ - AI Research Center (XARC), 9F, Tower A, Dongsheng Building, No.8, Zhongguancun East Road, Haidian District, Beijing 100083, P.R. China 
Fig. S1. The correlations between the MM and SQM (PM6-D3H4X) energetics calculated from 25 ns trajectories generated at $600 \mathrm{~K}$ (left) and $300 \mathrm{~K}$ (right) in vacuo for the host and 7 guest molecules. The sampling interval is $5 \mathrm{ps}$ and there are 5000 independent configurations in total. The RMSE and MAE of the original parameter set and the newly fitted force-matching set are also presented. Note that the protonated and deprotonated Ketamine are fitted separately.
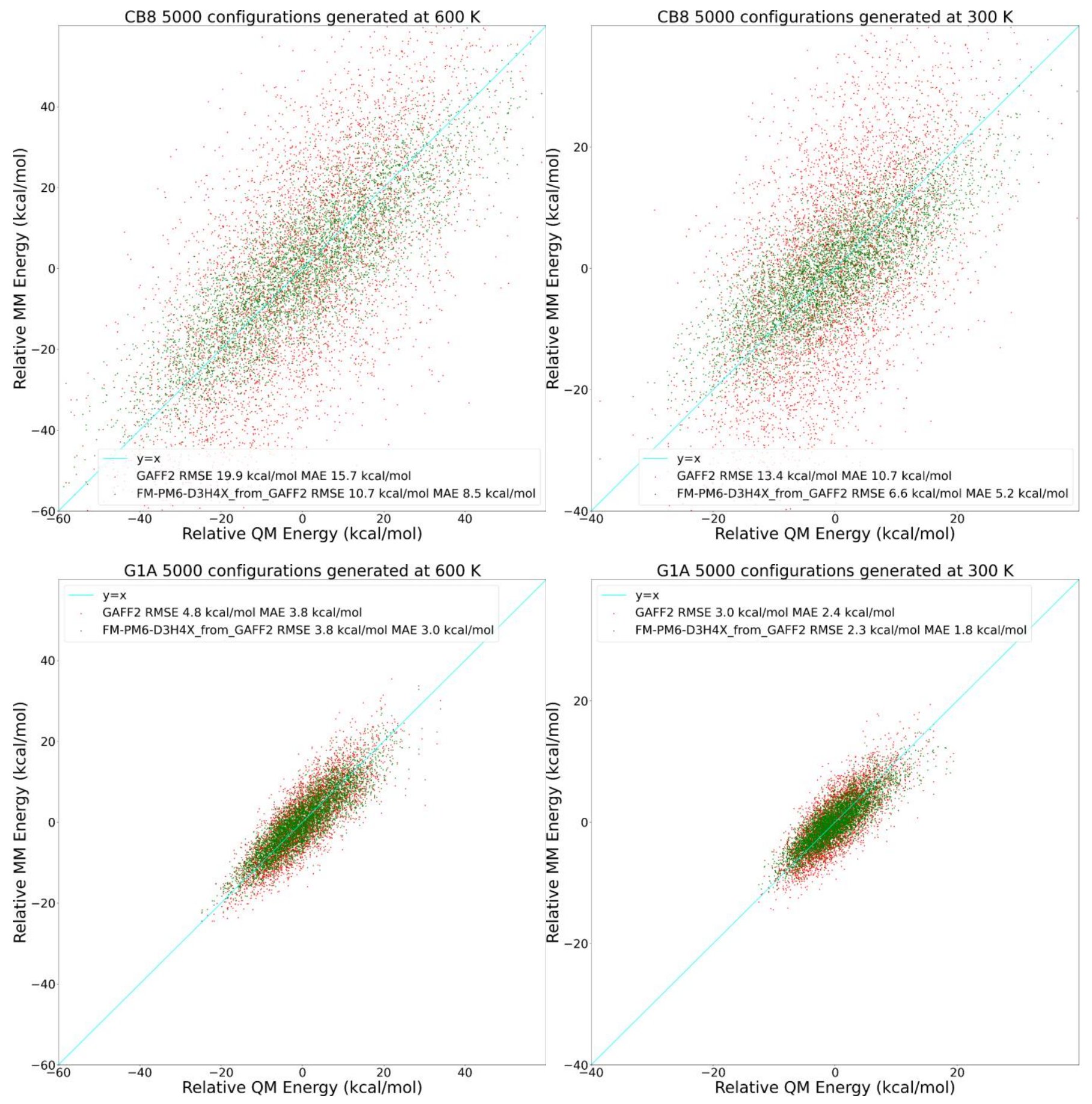

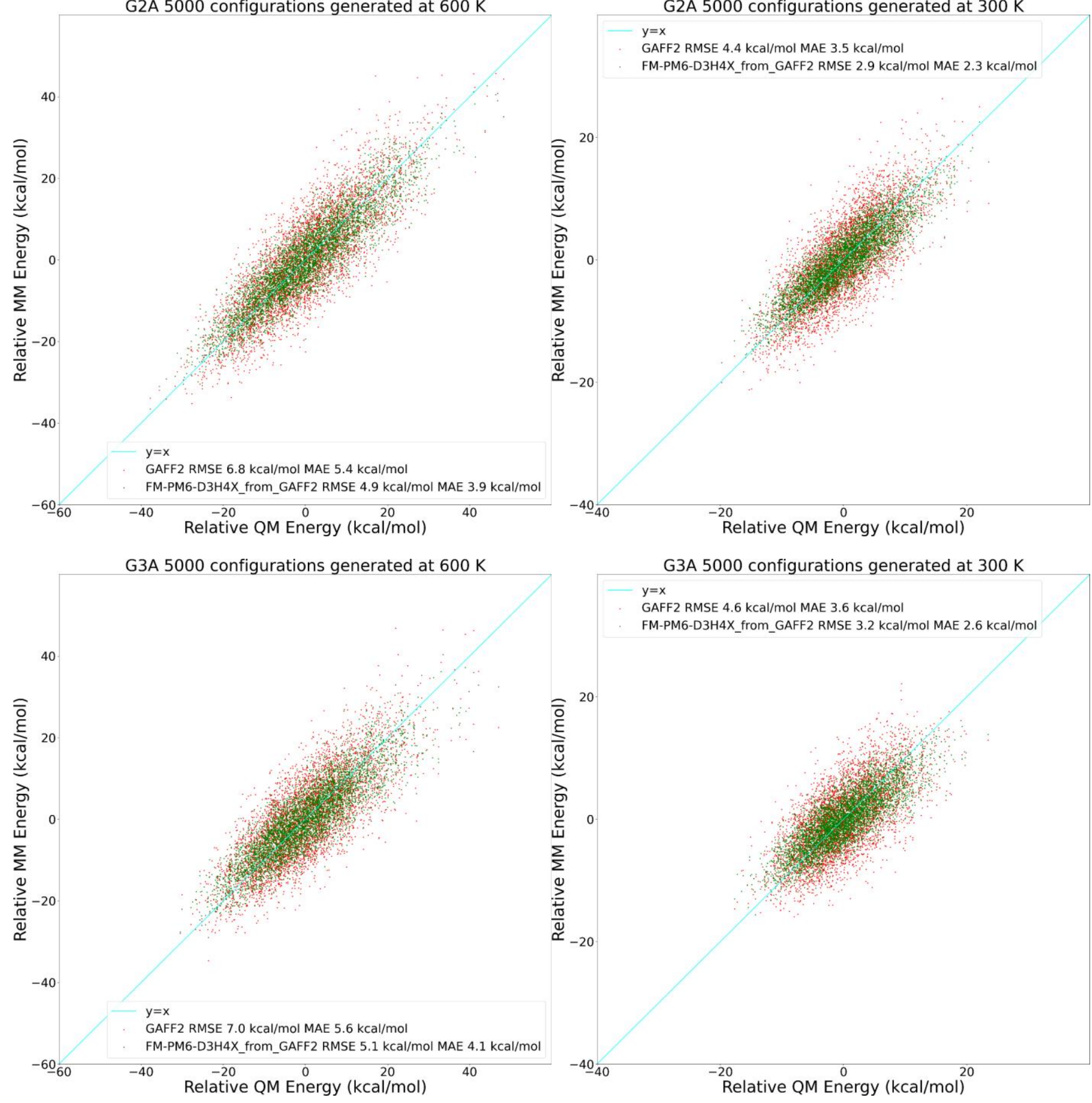

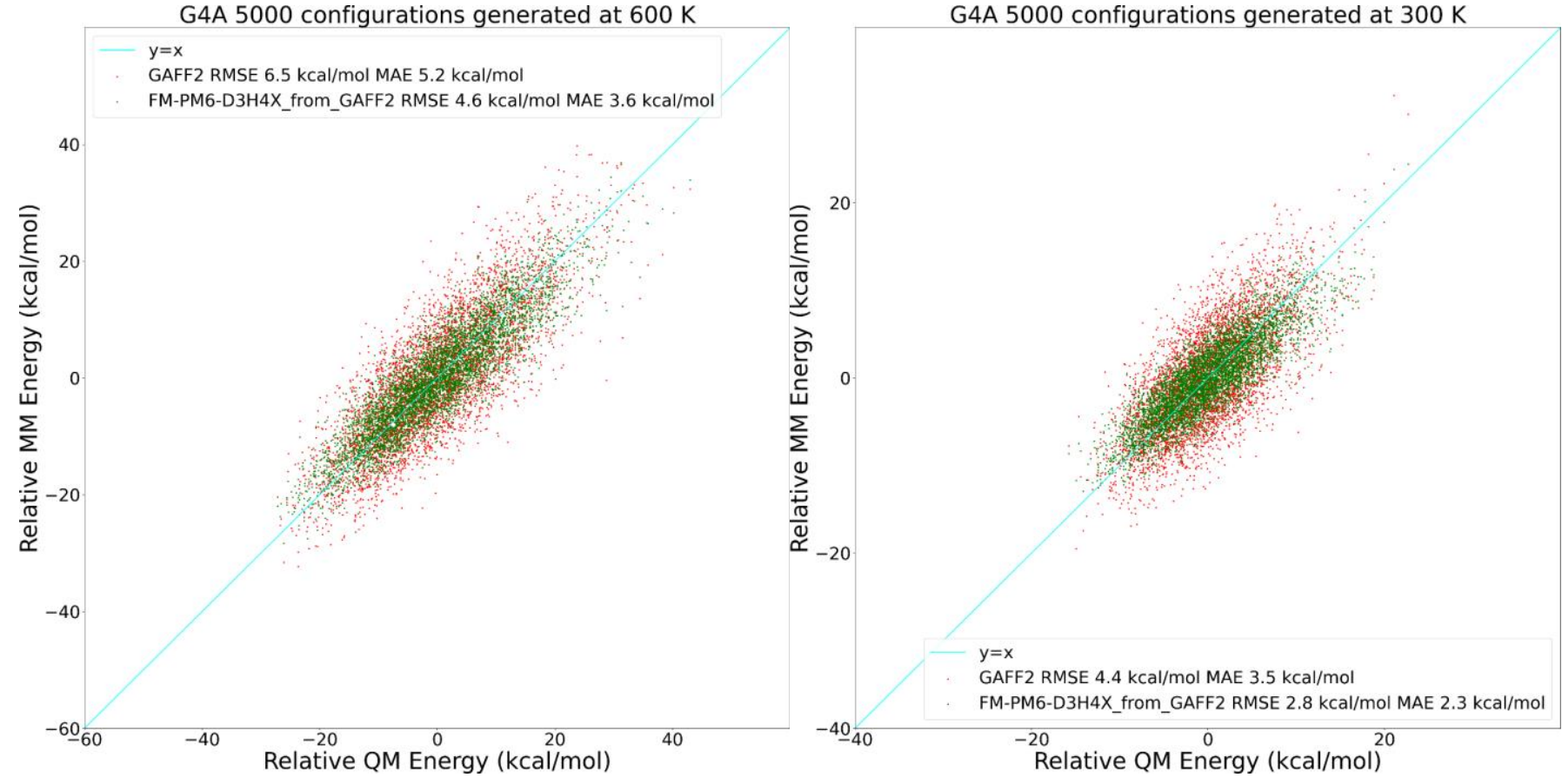

G5A 5000 configurations generated at $600 \mathrm{~K}$
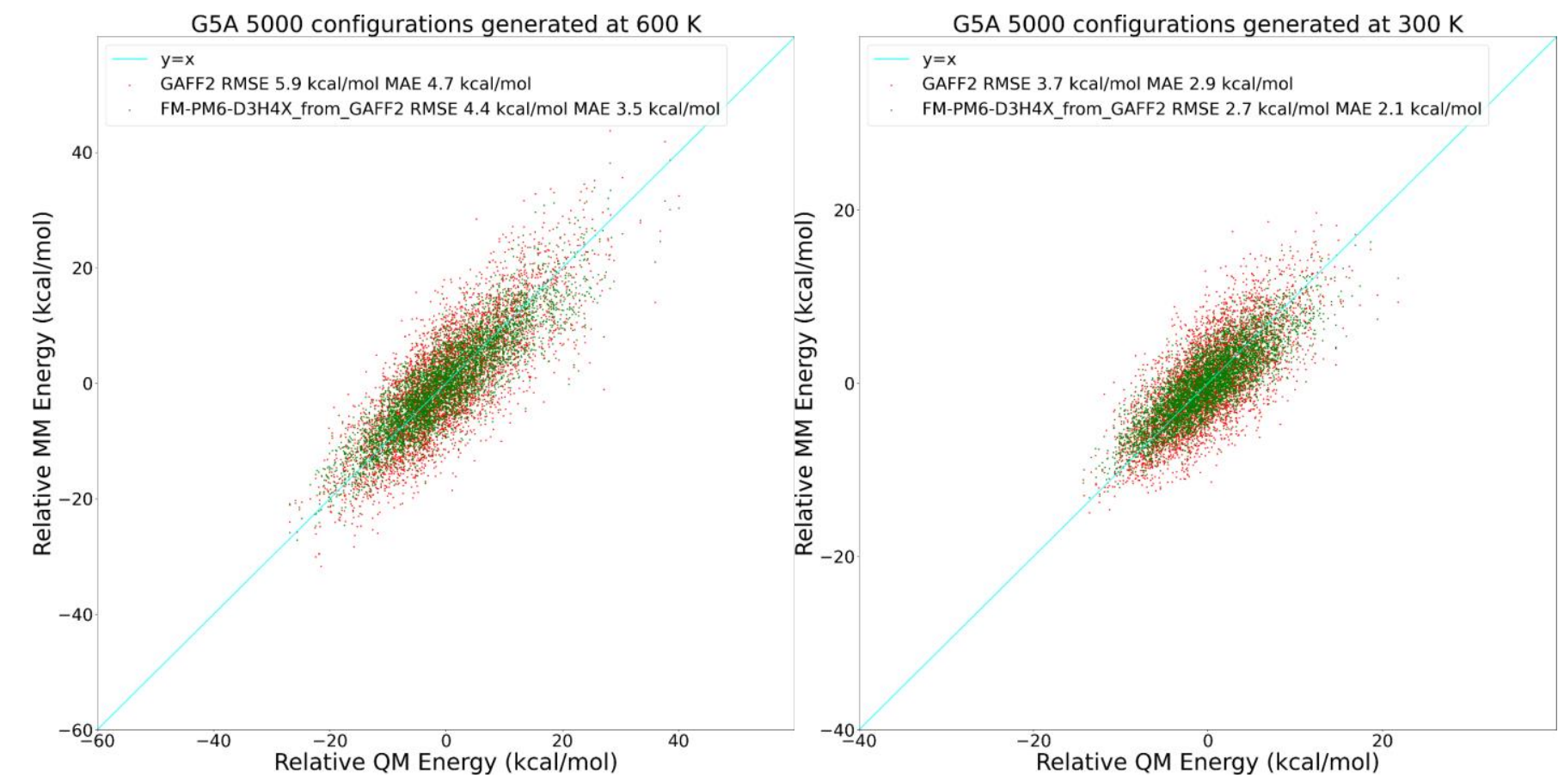

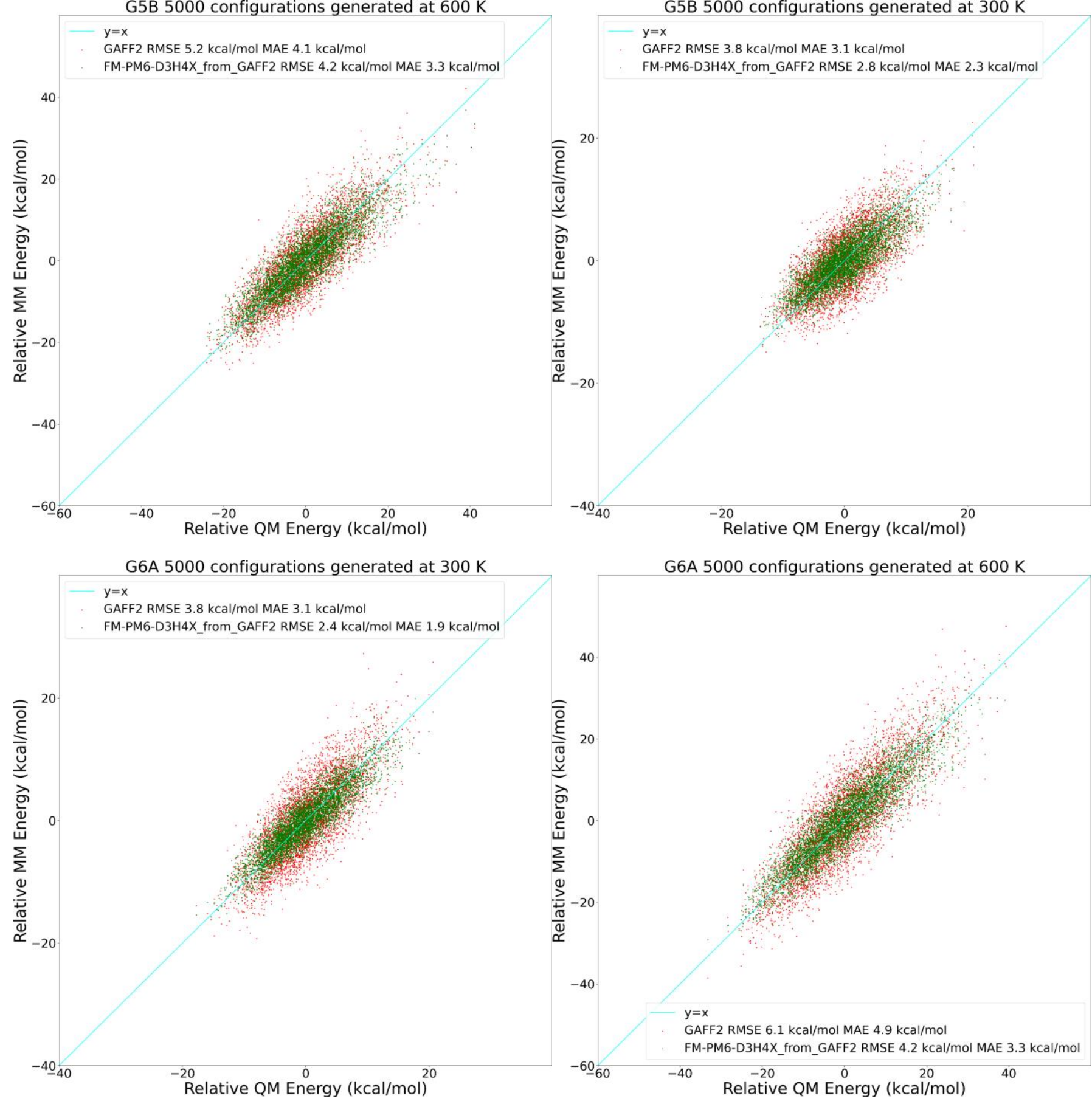

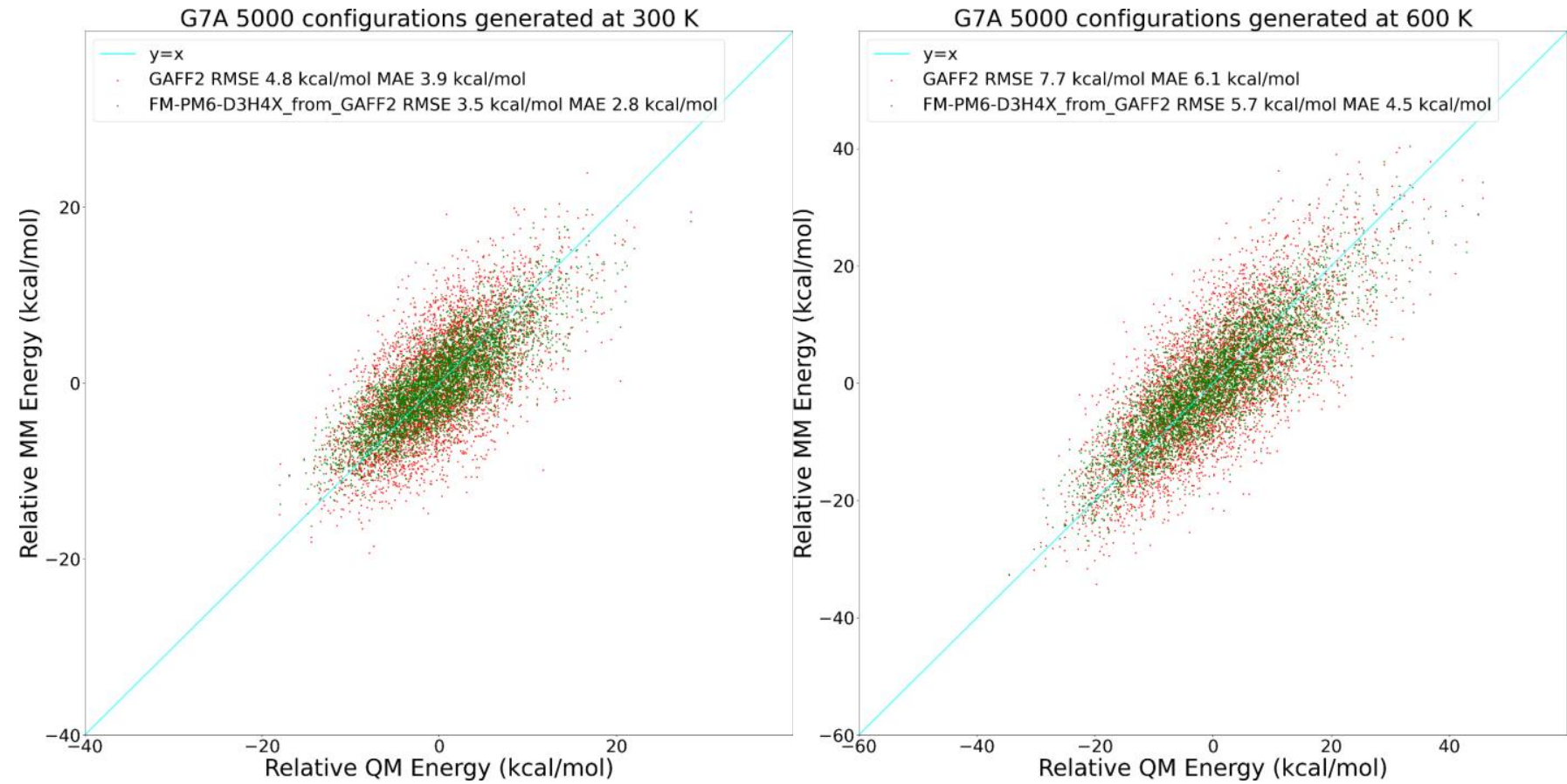
Fig. S2. The time series of the errors of atomic forces $\left(\left\|\Delta \mathbf{F}_{i}\right\|_{2}\right.$ for the $i$ th atom) under the original GAFF2 and the refitted FM-PM6_from_GAFF2 parameter set calculated from $25 \mathrm{~ns}$ trajectories generated at $600 \mathrm{~K}$ and $300 \mathrm{~K}$ in vacuo for the host and 7 guest molecules. The protonated and deprotonated forms of Ketamine are parameterized and thus tested separately. The sampling interval is 5 ps and there are 5000 independent configurations in total. Red dots for the force errors larger than $50 \mathrm{kcal} /(\mathrm{mol} \cdot \AA)$, green for force errors larger than $30 \mathrm{kcal} /(\mathrm{mol} \cdot \AA)$, blue for errors larger than $10 \mathrm{kcal} /(\mathrm{mol} \cdot \AA)$, and white for the other small-error points. The overall RMSEs of atomic forces of each molecule in $\mathrm{kcal} /(\mathrm{mol} \cdot \AA \cdot$ atom $)$ under the original GAFF2 and the newly obtained FM-PM6 parameter sets are also given.

\section{$>50 \mathrm{kcal} /(\mathrm{mol} \cdot \AA)>30 \mathrm{kcal} /(\mathrm{mol} \cdot \AA)>10 \mathrm{kcal} /(\mathrm{mol} \cdot \AA)$}
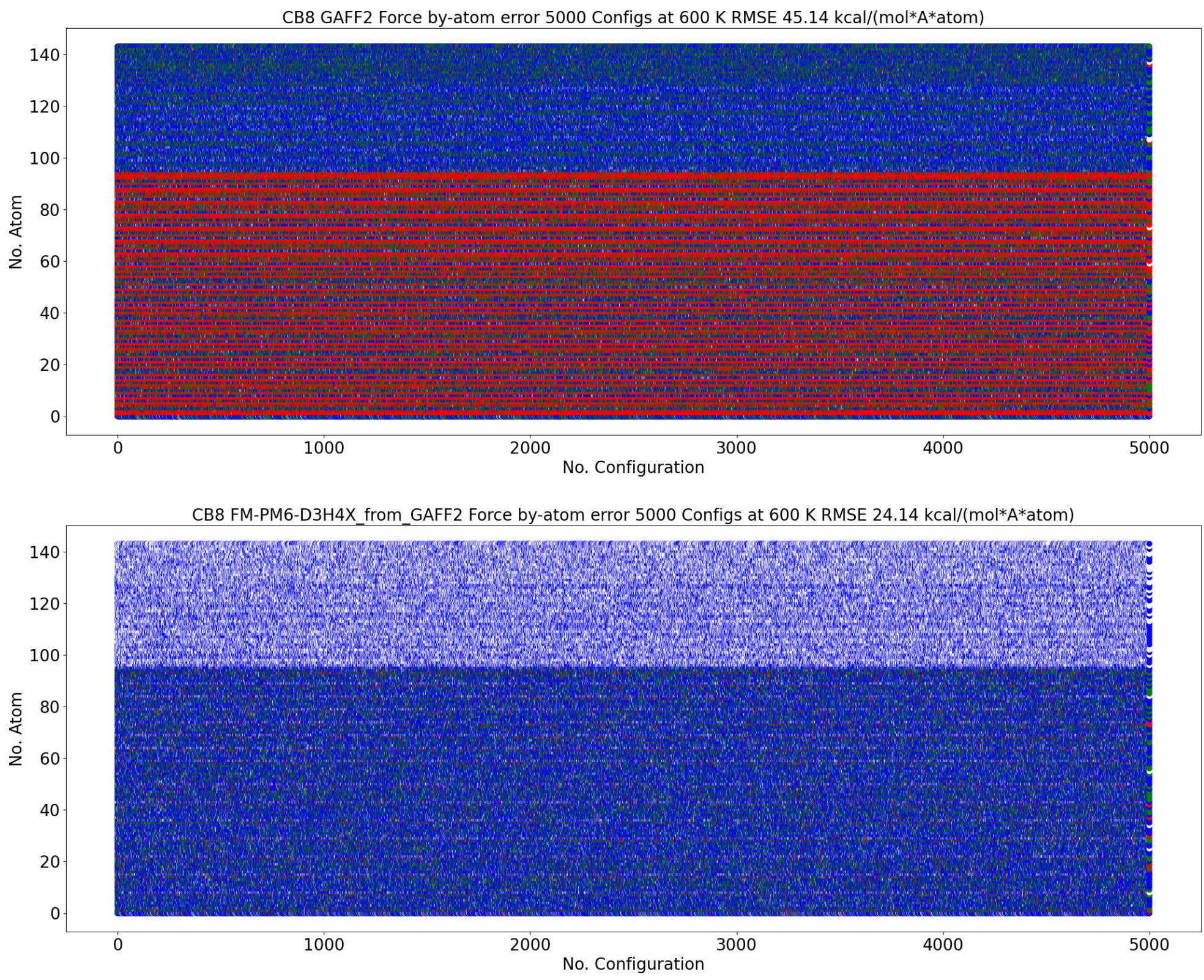
CB8 GAFF2 Force by-atom error 5000 Configs at $300 \mathrm{~K}$ RMSE $40.05 \mathrm{kcal} /\left(\mathrm{mol} * \mathrm{~A}^{*}\right.$ atom)

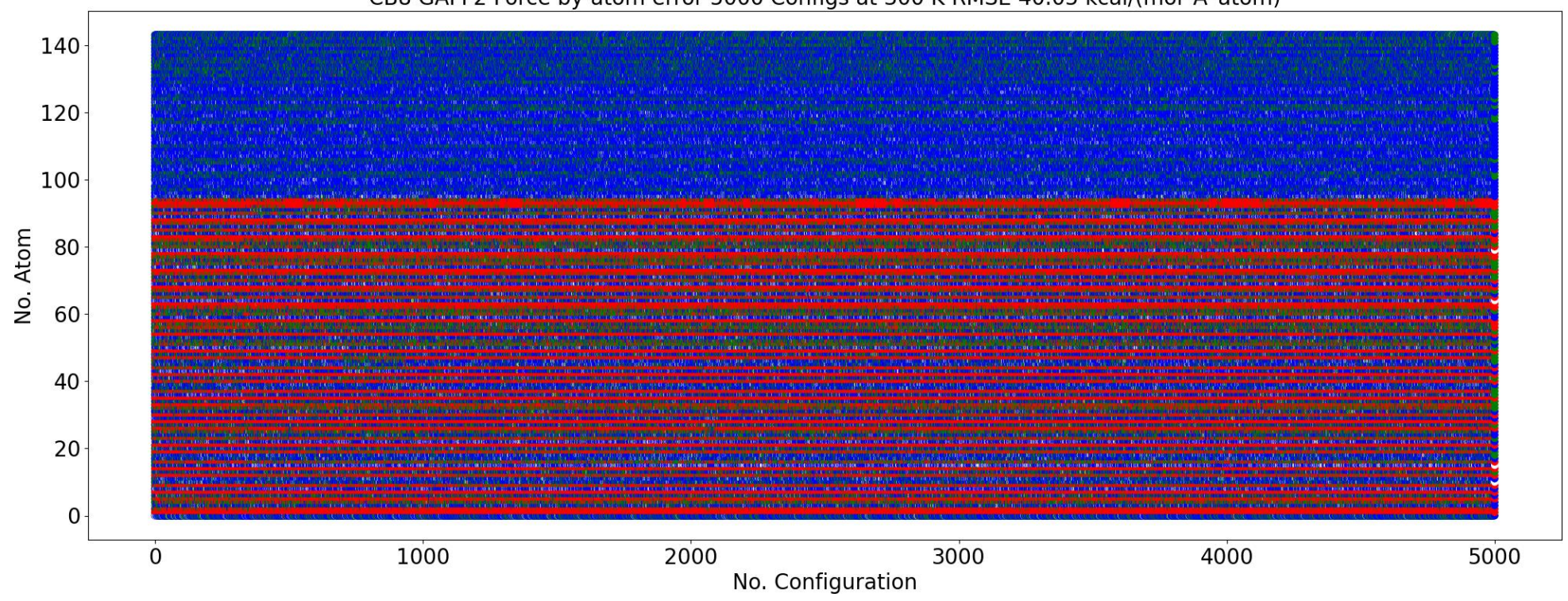

CB8 FM-PM6-D3H4X_from_GAFF2 Force by-atom error 5000 Configs at $300 \mathrm{~K}$ RMSE $17.87 \mathrm{kcal} /(\mathrm{mol} * A * a t o m)$

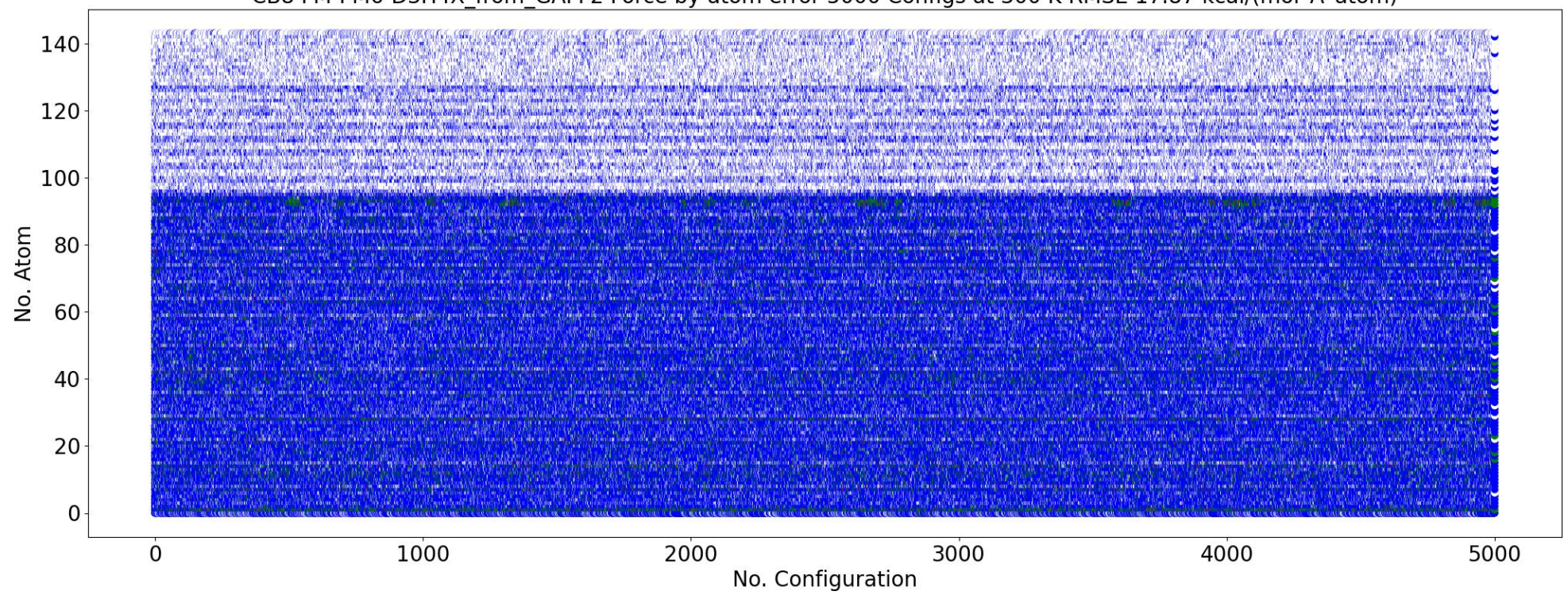




\section{$>50 \mathrm{kcal} /(\mathrm{mol} \cdot \AA)>30 \mathrm{kcal} /(\mathrm{mol} \cdot \AA)>10 \mathrm{kcal} /(\mathrm{mol} \cdot \AA)$}

G1A GAFF2 Force by-atom error 5000 Configs at $600 \mathrm{~K} \mathrm{RMSE} 29.11 \mathrm{kcal} /\left(\mathrm{mol} * \mathrm{~A}^{*}\right.$ atom)

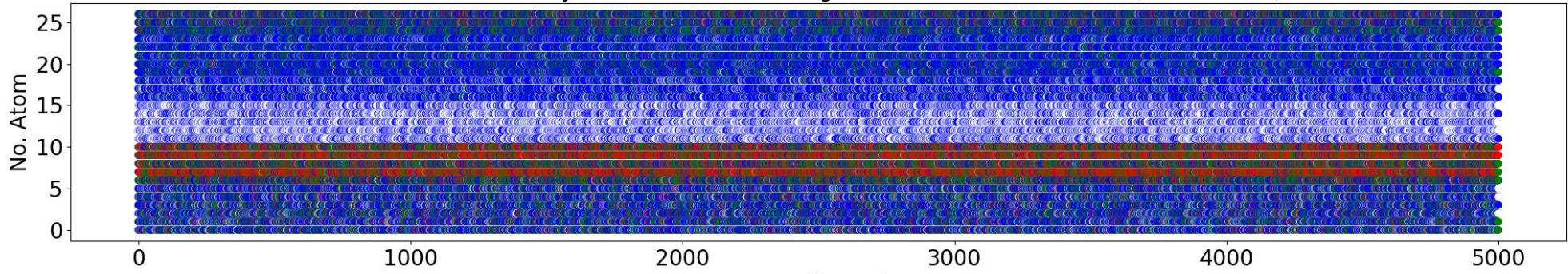

G1A FM-PM6-D3H4X_from_GAFF2 Force by-atom error 5000 Configs at $600 \mathrm{~K}$ RMSE $19.41 \mathrm{kcal} /\left(\mathrm{mol} * A^{*}\right.$ atom)

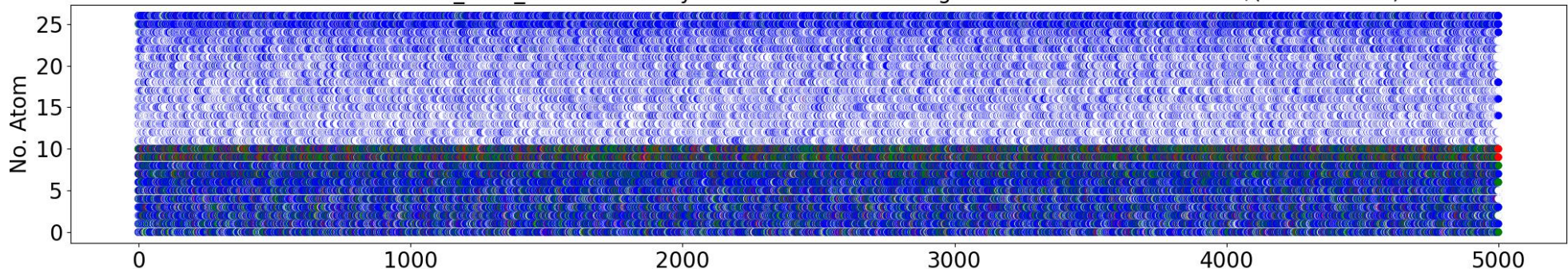

G1A GAFF2 Force by-atom error 5000 Configs at $300 \mathrm{~K}$ RMSE $24.23 \mathrm{kcal} /\left(\mathrm{mol} * \mathrm{~A}^{*}\right.$ atom)

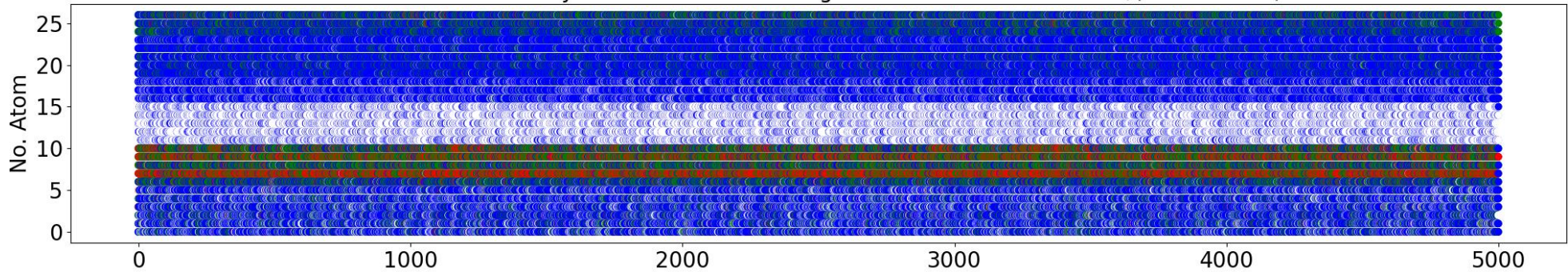

G1A FM-PM6-D3H4X_from_GAFF2 Force by-atom error 5000 Configs at $300 \mathrm{~K}$ RMSE $14.18 \mathrm{kcal} /\left(\mathrm{mol} * \mathrm{~A}^{*}\right.$ atom)

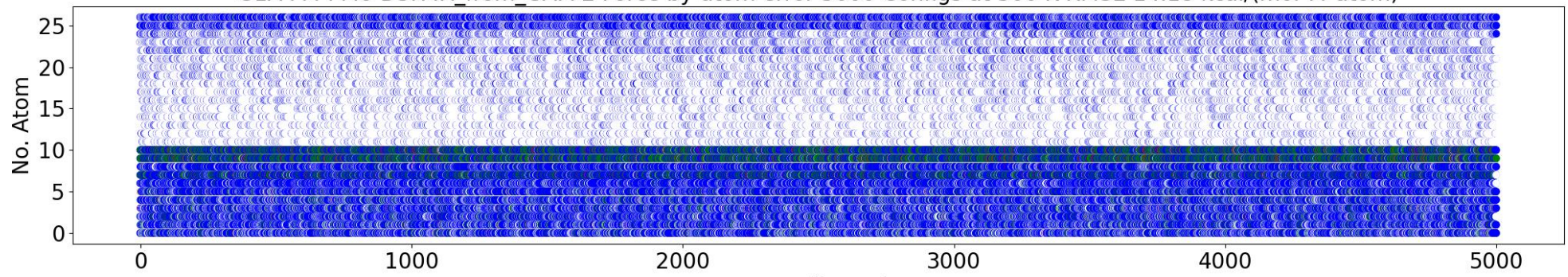




\section{$>50 \mathrm{kcal} /(\mathrm{mol} \cdot \AA)>30 \mathrm{kcal} /(\mathrm{mol} \cdot \AA)>10 \mathrm{kcal} /(\mathrm{mol} \cdot \AA)$}

G2A GAFF2 Force by-atom error 5000 Configs at $600 \mathrm{~K}$ RMSE $28.85 \mathrm{kcal} /\left(\mathrm{mol} * \mathrm{~A}^{*}\right.$ atom)

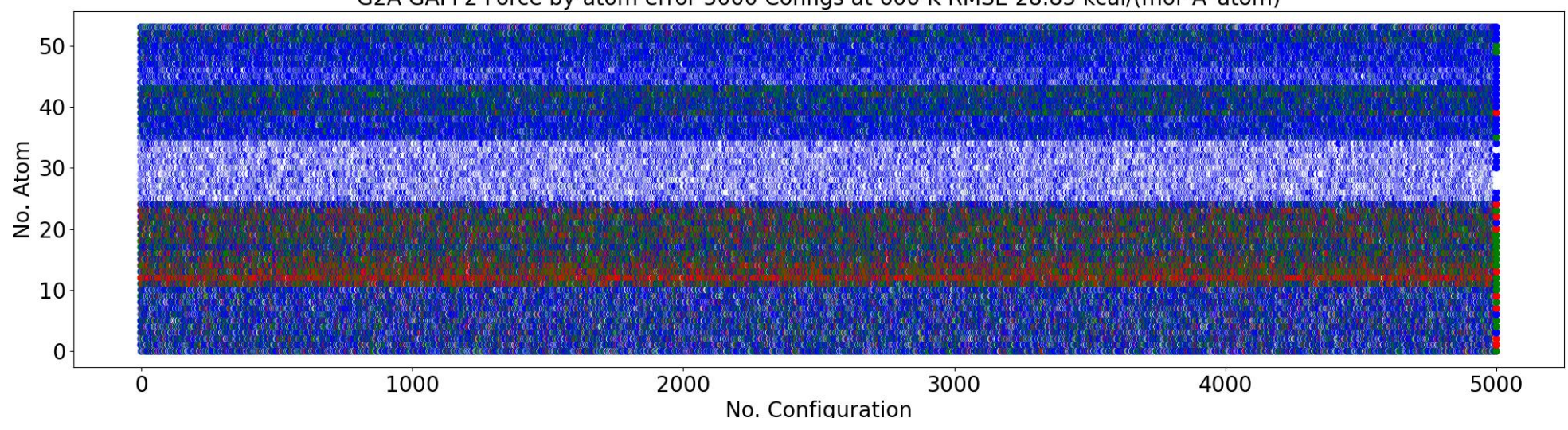

G2A FM-PM6-D3H4X_from_GAFF2 Force by-atom error 5000 Configs at $600 \mathrm{~K}$ RMSE $19.15 \mathrm{kcal} /(\mathrm{mol}$ *A*atom)

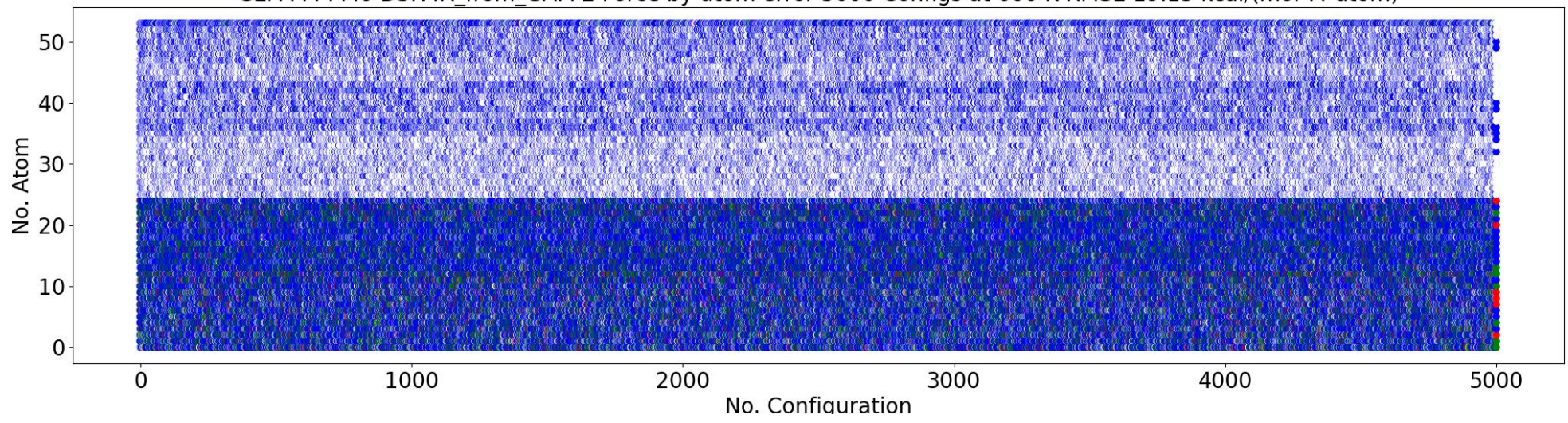

G2A GAFF2 Force by-atom error 5000 Configs at $300 \mathrm{~K}$ RMSE $24.10 \mathrm{kcal} /\left(\mathrm{mol} * \mathrm{~A}^{*}\right.$ atom)

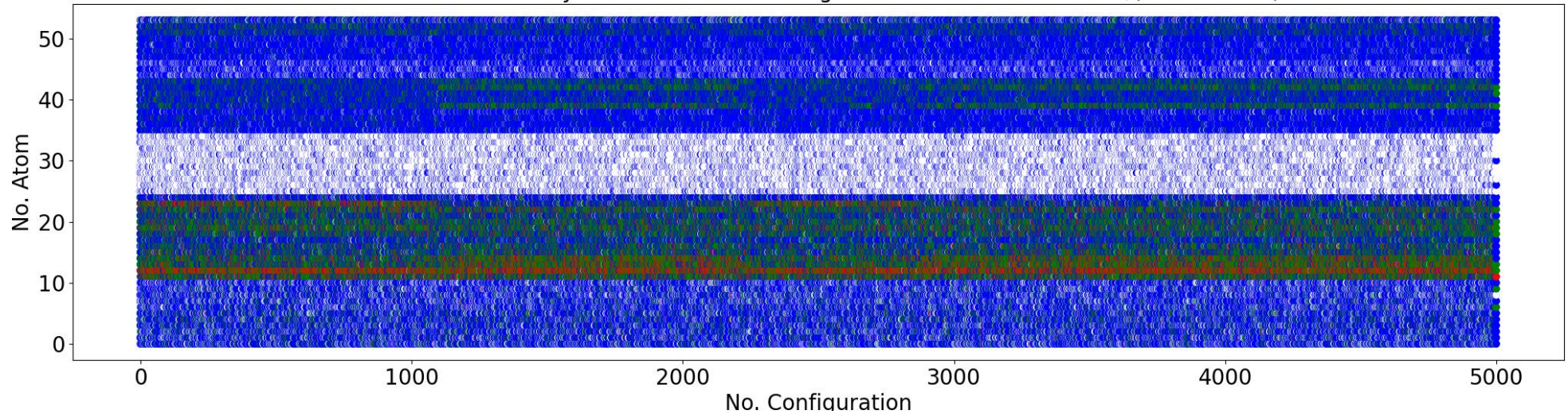

G2A FM-PM6-D3H4X_from_GAFF2 Force by-atom error 5000 Configs at $300 \mathrm{~K}$ RMSE $13.64 \mathrm{kcal} /\left(\mathrm{mol} * A^{*}\right.$ atom)

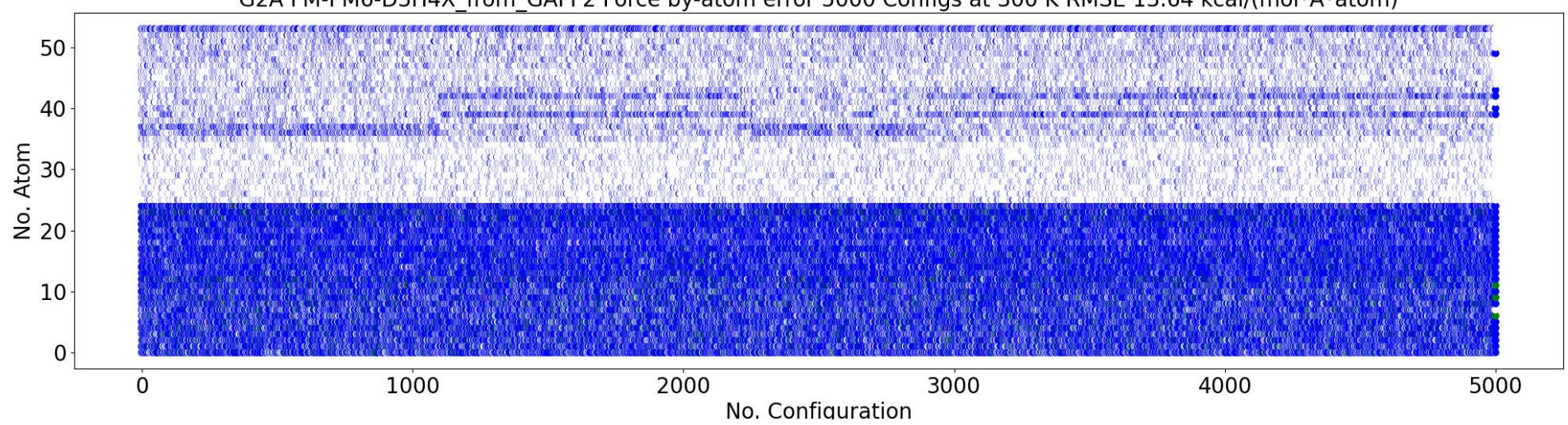




\section{$>50 \mathrm{kcal} /(\mathrm{mol} \cdot \AA)>30 \mathrm{kcal} /(\mathrm{mol} \cdot \AA)>10 \mathrm{kcal} /(\mathrm{mol} \cdot \AA)$}

G3A GAFF2 Force by-atom error 5000 Configs at $600 \mathrm{~K}$ RMSE $32.21 \mathrm{kcal} /\left(\mathrm{mol} * A^{*}\right.$ atom)

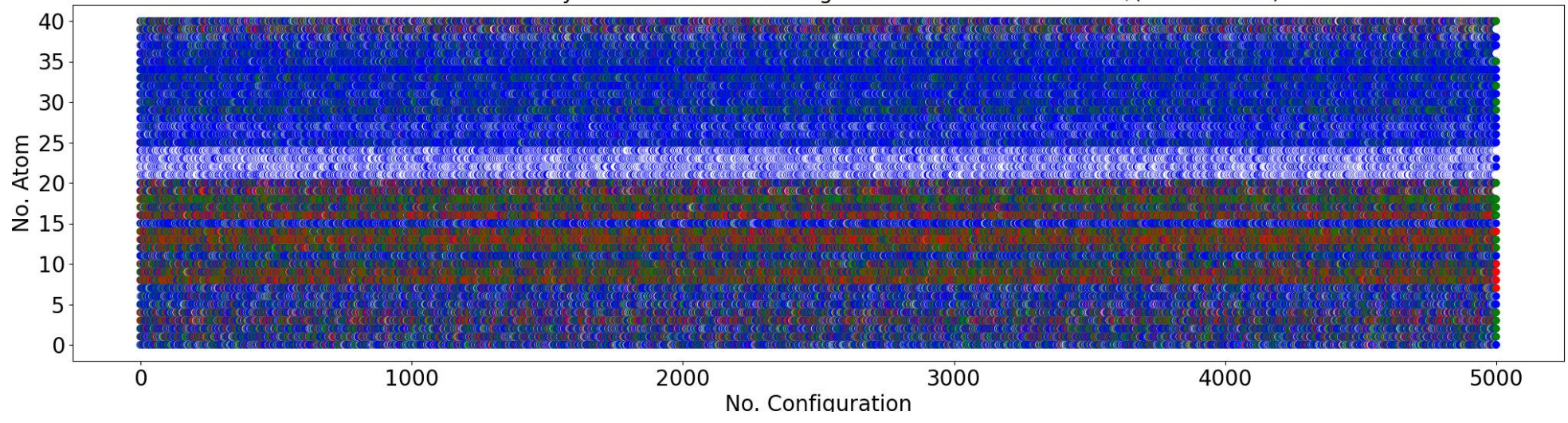

G3A FM-PM6-D3H4X_from_GAFF2 Force by-atom error 5000 Configs at $600 \mathrm{~K}$ RMSE $21.85 \mathrm{kcal} /(\mathrm{mol}$ *A*atom)

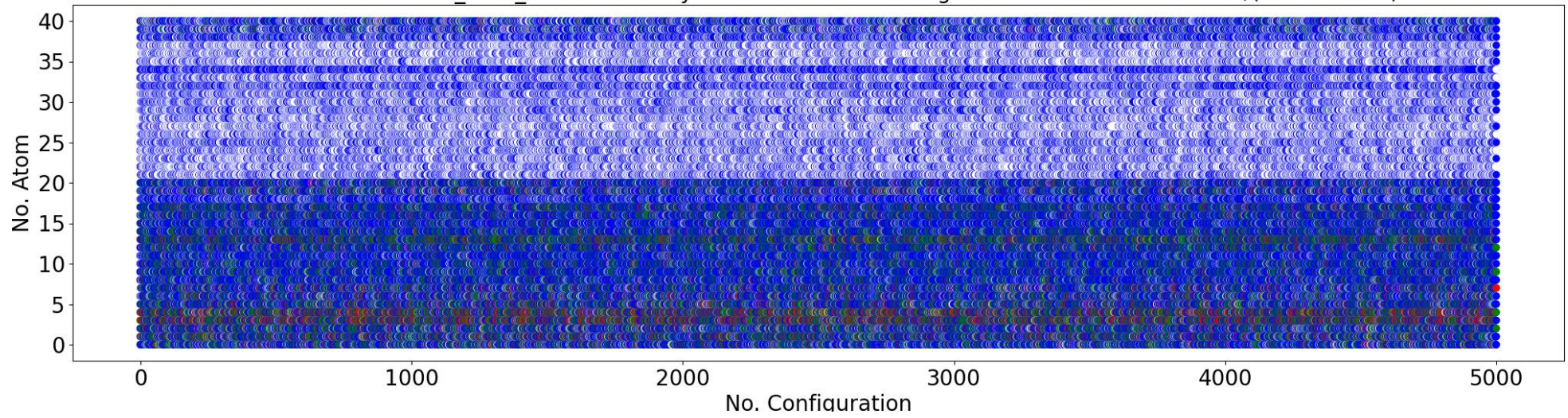

G3A GAFF2 Force by-atom error 5000 Configs at $300 \mathrm{~K}$ RMSE $27.63 \mathrm{kcal} /\left(\mathrm{mol} * \mathrm{~A}^{*}\right.$ atom)

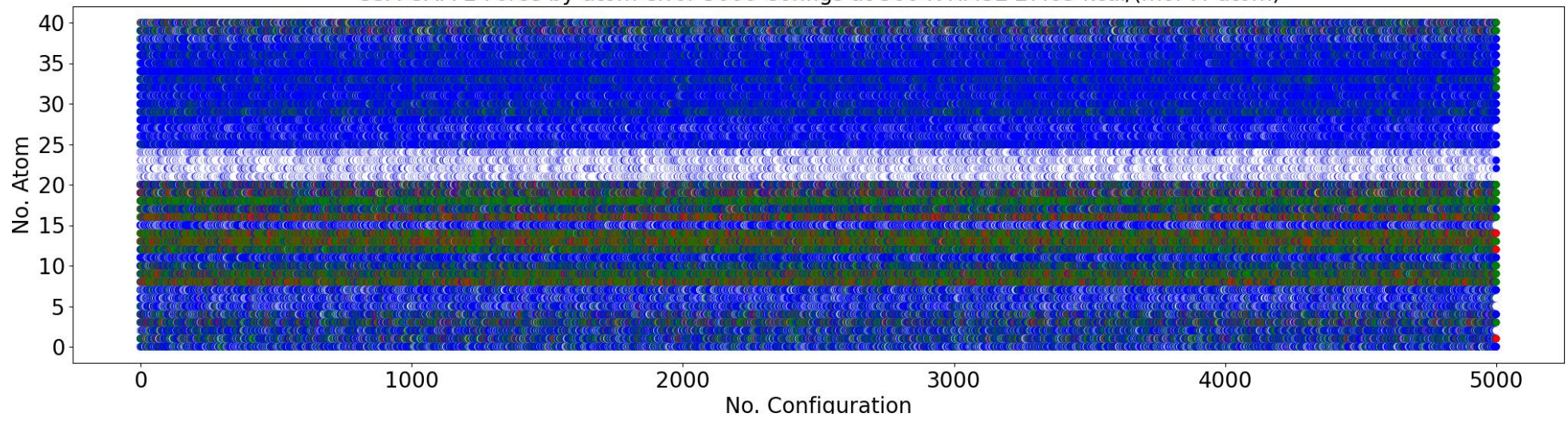

G3A FM-PM6-D3H4X_from_GAFF2 Force by-atom error 5000 Configs at $300 \mathrm{~K}$ RMSE $16.38 \mathrm{kcal} /\left(\mathrm{mol} * A^{*}\right.$ atom)

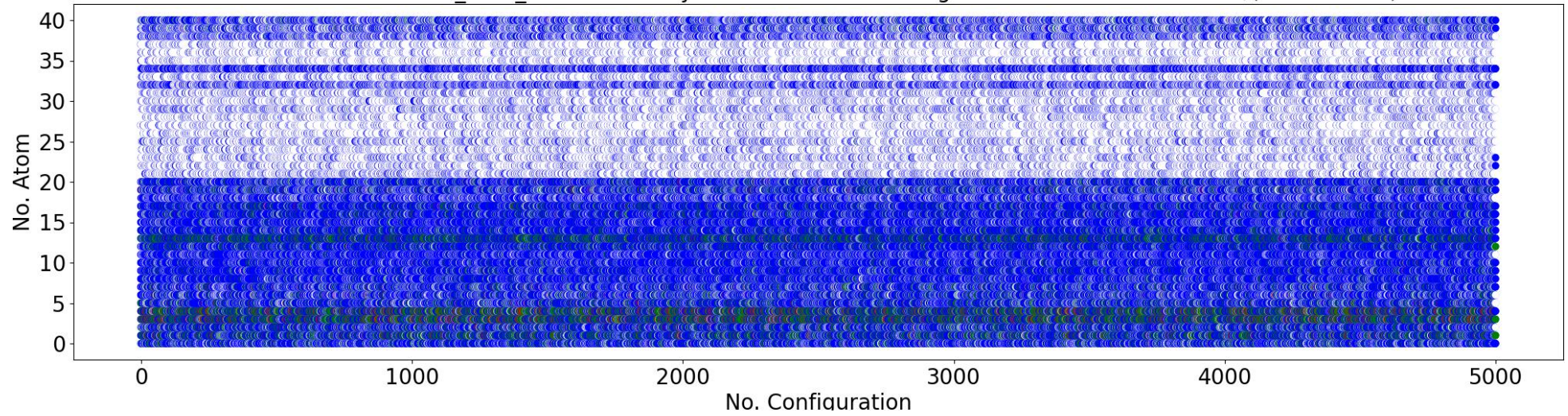




\section{$>50 \mathrm{kcal} /(\mathrm{mol} \cdot \AA)>30 \mathrm{kcal} /(\mathrm{mol} \cdot \AA)>10 \mathrm{kcal} /(\mathrm{mol} \cdot \AA)$}

G4A GAFF2 Force by-atom error 5000 Configs at $600 \mathrm{~K}$ RMSE $32.50 \mathrm{kcal} /\left(\mathrm{mol} * A^{*}\right.$ atom)

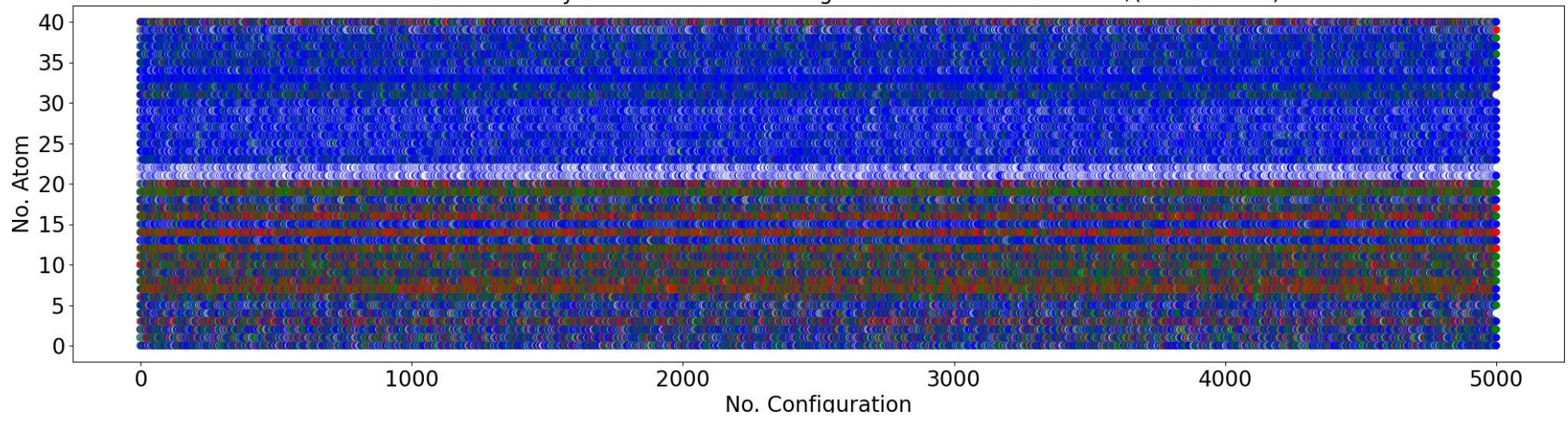

G4A FM-PM6-D3H4X_from_GAFF2 Force by-atom error 5000 Configs at $600 \mathrm{~K}$ RMSE $20.97 \mathrm{kcal} /(\mathrm{mol}$ *A*atom)

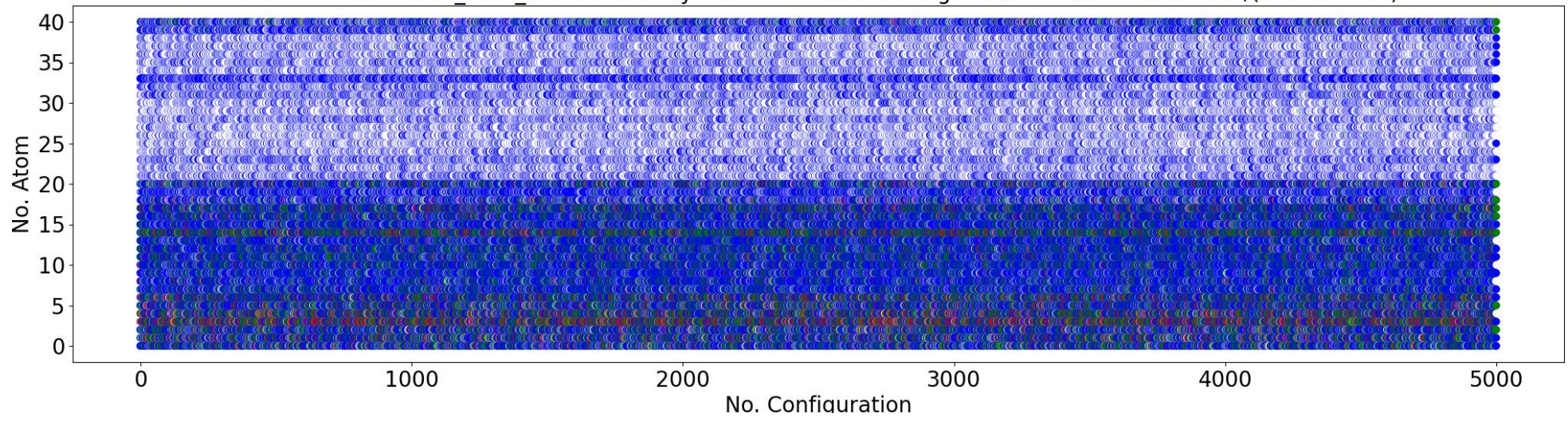

G4A GAFF2 Force by-atom error 5000 Configs at $300 \mathrm{~K}$ RMSE $28.11 \mathrm{kcal} /\left(\mathrm{mol} * \mathrm{~A}^{*}\right.$ atom)

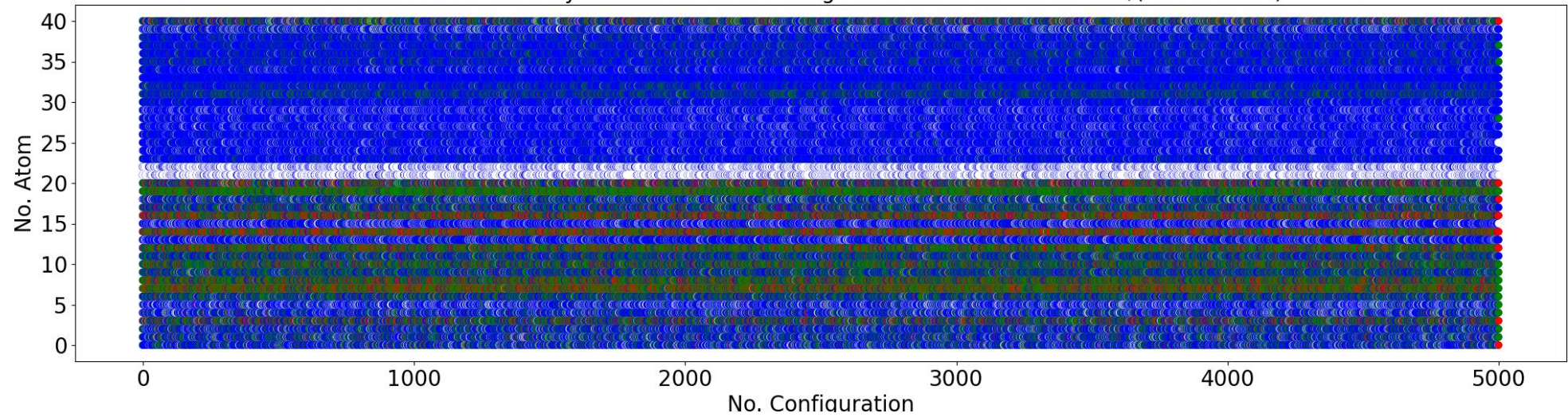

G4A FM-PM6-D3H4X_from_GAFF2 Force by-atom error 5000 Configs at $300 \mathrm{~K}$ RMSE $15.65 \mathrm{kcal} /\left(\mathrm{mol} * \mathrm{~A}^{*}\right.$ atom)

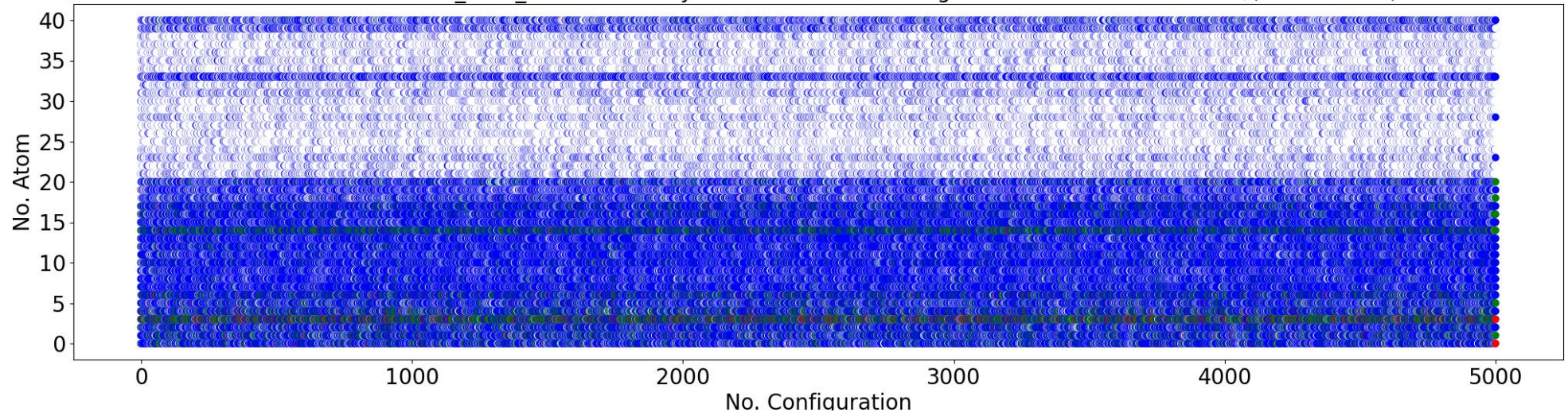




\section{$>50 \mathrm{kcal} /(\mathrm{mol} \cdot \AA)>30 \mathrm{kcal} /(\mathrm{mol} \cdot \AA)>10 \mathrm{kcal} /(\mathrm{mol} \cdot \AA)$}

G5A GAFF2 Force by-atom error 5000 Configs at $600 \mathrm{~K} \mathrm{RMSE} 30.78 \mathrm{kcal} /\left(\mathrm{mol} * \mathrm{~A}^{*}\right.$ atom)

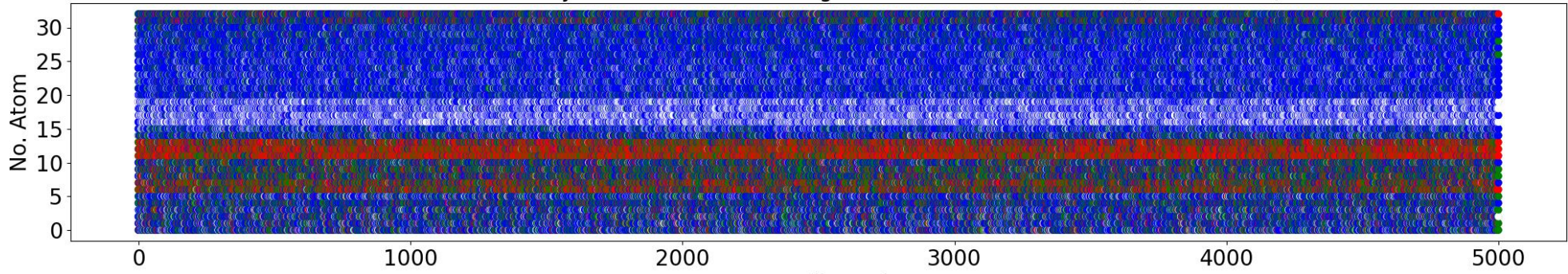

G5A FM-PM6-D3H4X_from_GAFF2 Force by-atom error 5000 Configs at $600 \mathrm{~K}$ RMSE $20.82 \mathrm{kcal} /\left(\mathrm{mol} * A^{*}\right.$ atom)

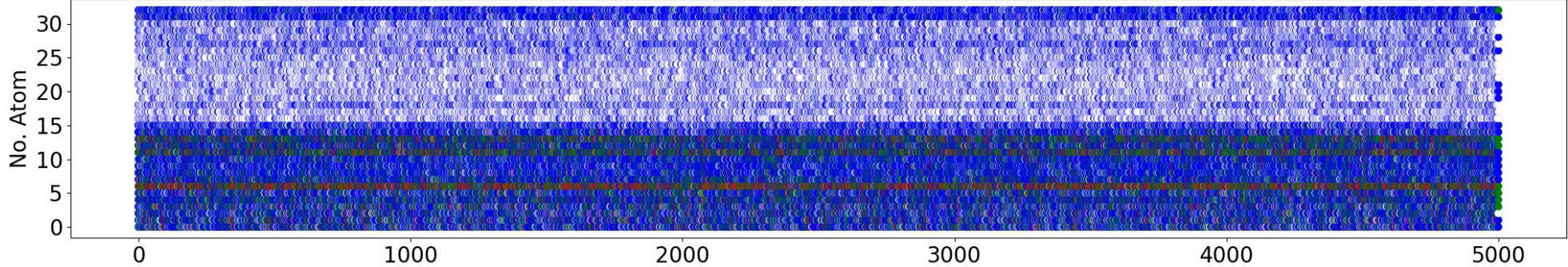

G5A GAFF2 Force by-atom error 5000 Configs at $300 \mathrm{~K}$ RMSE $26.17 \mathrm{kcal} /\left(\mathrm{mol} * \mathrm{~A}^{*}\right.$ atom)

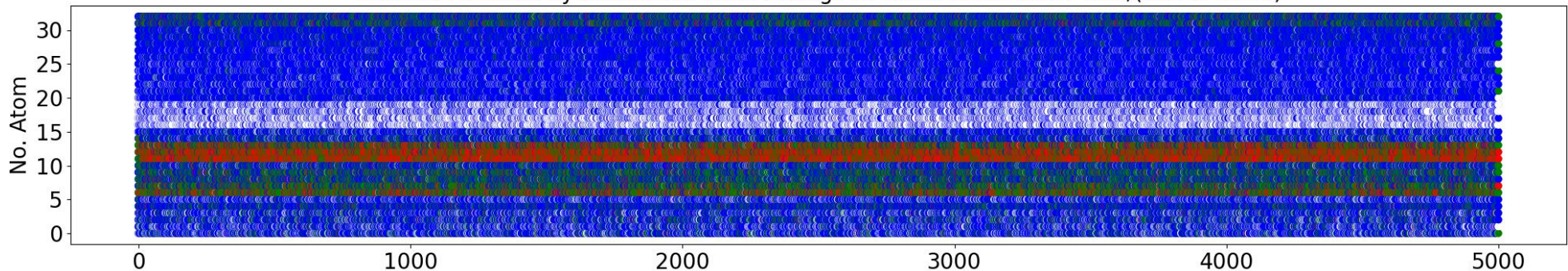

G5A FM-PM6-D3H4X_from_GAFF2 Force by-atom error 5000 Configs at $300 \mathrm{~K}$ RMSE $15.43 \mathrm{kcal} /\left(\mathrm{mol} * A^{*}\right.$ atom)

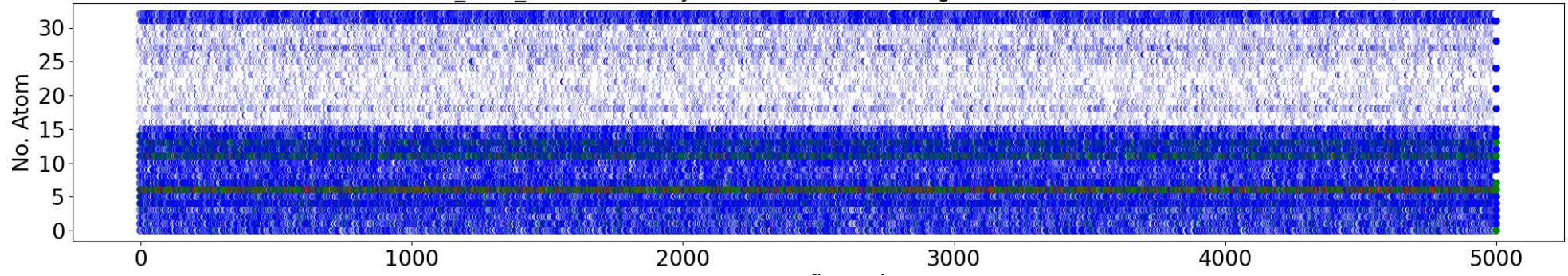




\section{$>50 \mathrm{kcal} /(\mathrm{mol} \cdot \AA)>30 \mathrm{kcal} /(\mathrm{mol} \cdot \AA)>10 \mathrm{kcal} /(\mathrm{mol} \cdot \AA)$}

G5B GAFF2 Force by-atom error 5000 Configs at $600 \mathrm{~K}$ RMSE $26.59 \mathrm{kcal} /\left(\mathrm{mol} * \mathrm{~A}^{*}\right.$ atom)

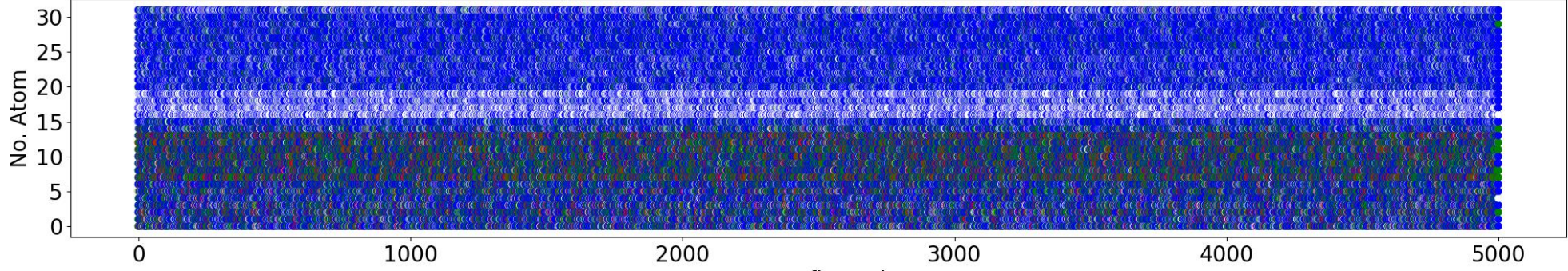

G5B FM-PM6-D3H4X_from_GAFF2 Force by-atom error 5000 Configs at $600 \mathrm{~K}$ RMSE $20.01 \mathrm{kcal} /\left(\mathrm{mol} * A^{*}\right.$ atom)

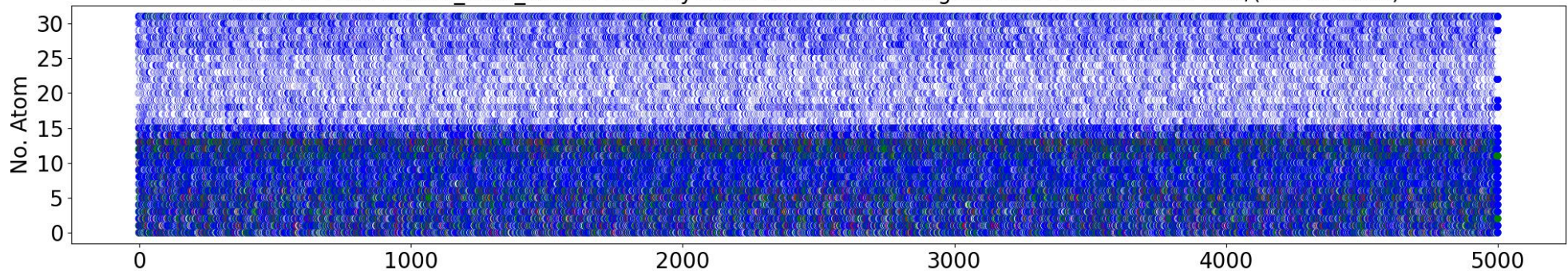

G5B GAFF2 Force by-atom error 5000 Configs at $300 \mathrm{~K}$ RMSE $21.43 \mathrm{kcal} /(\mathrm{mol} * \mathrm{~A} *$ atom)

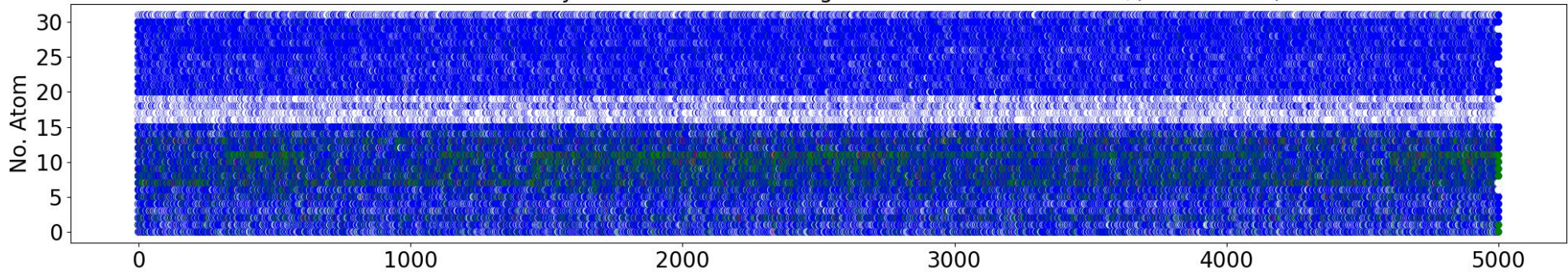

G5B FM-PM6-D3H4X_from_GAFF2 Force by-atom error 5000 Configs at $300 \mathrm{~K}$ RMSE $14.43 \mathrm{kcal} /\left(\mathrm{mol} * A^{*}\right.$ atom)

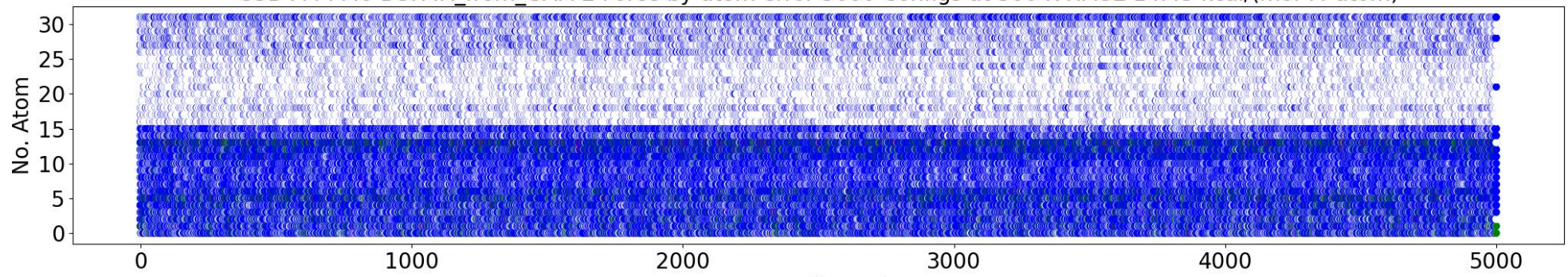




\section{$>50 \mathrm{kcal} /(\mathrm{mol} \cdot \AA)>30 \mathrm{kcal} /(\mathrm{mol} \cdot \AA)>10 \mathrm{kcal} /(\mathrm{mol} \cdot \AA)$}

G6A GAFF2 Force by-atom error 5000 Configs at $600 \mathrm{~K}$ RMSE $28.80 \mathrm{kcal} /\left(\mathrm{mol} * A^{*}\right.$ atom)

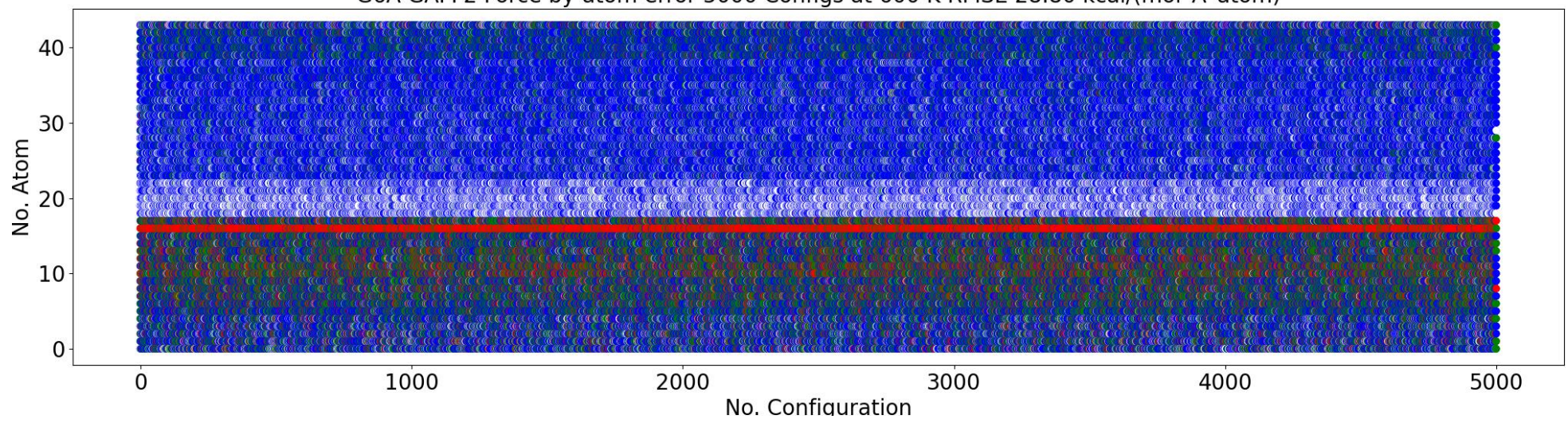

G6A FM-PM6-D3H4X_from_GAFF2 Force by-atom error 5000 Configs at $600 \mathrm{~K}$ RMSE $17.95 \mathrm{kcal} /(\mathrm{mol}$ *A*atom)

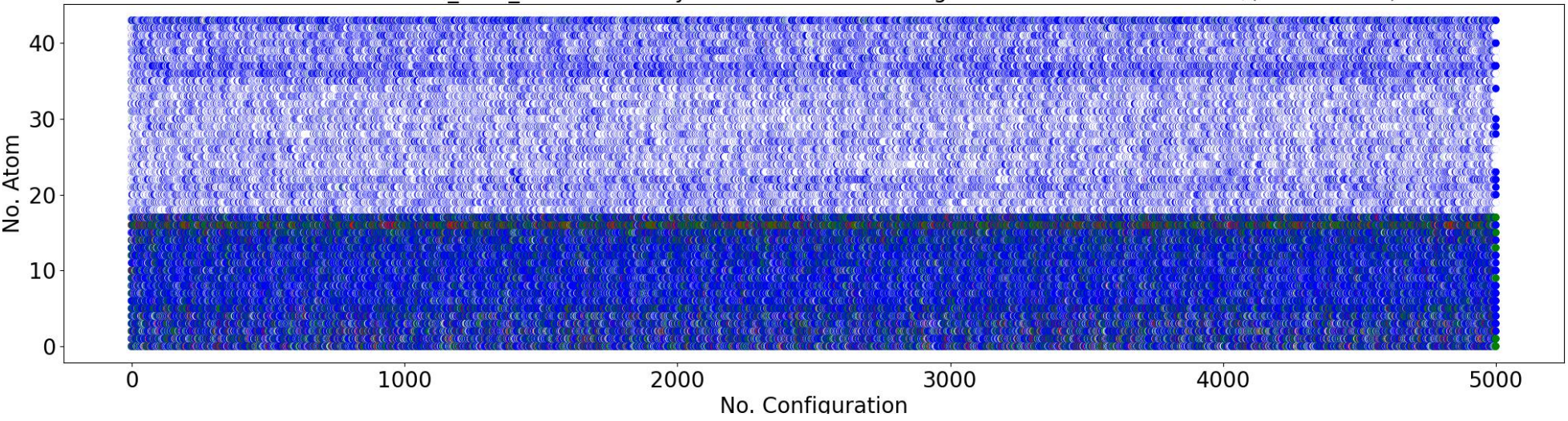

G6A GAFF2 Force by-atom error 5000 Configs at $300 \mathrm{~K}$ RMSE $24.05 \mathrm{kcal} /\left(\mathrm{mol} * \mathrm{~A}^{*}\right.$ atom)

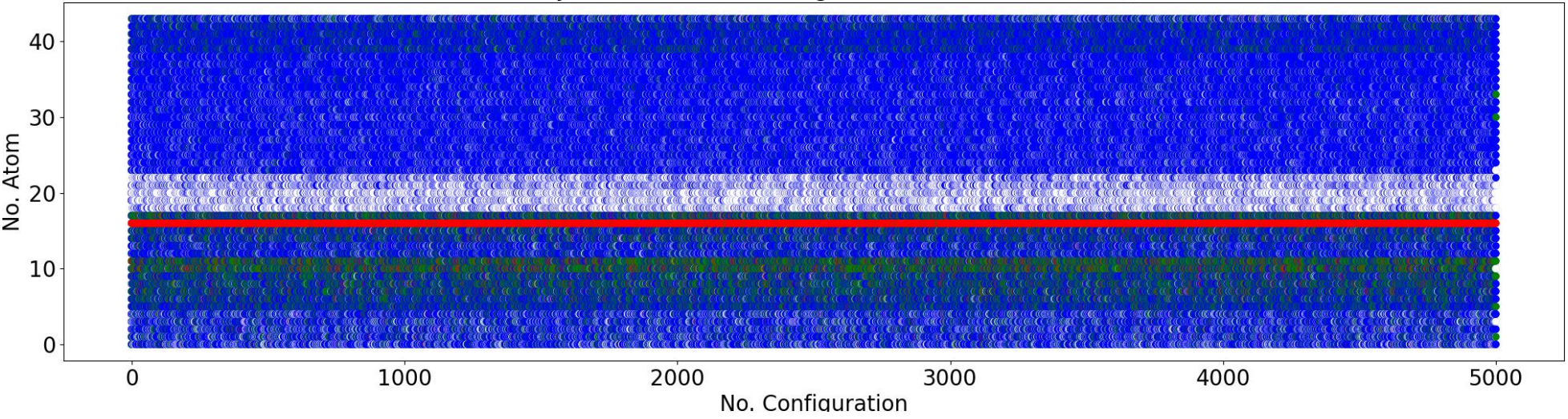

G6A FM-PM6-D3H4X_from_GAFF2 Force by-atom error 5000 Configs at $300 \mathrm{~K}$ RMSE $12.96 \mathrm{kcal} /(\mathrm{mol}$ *A*atom)

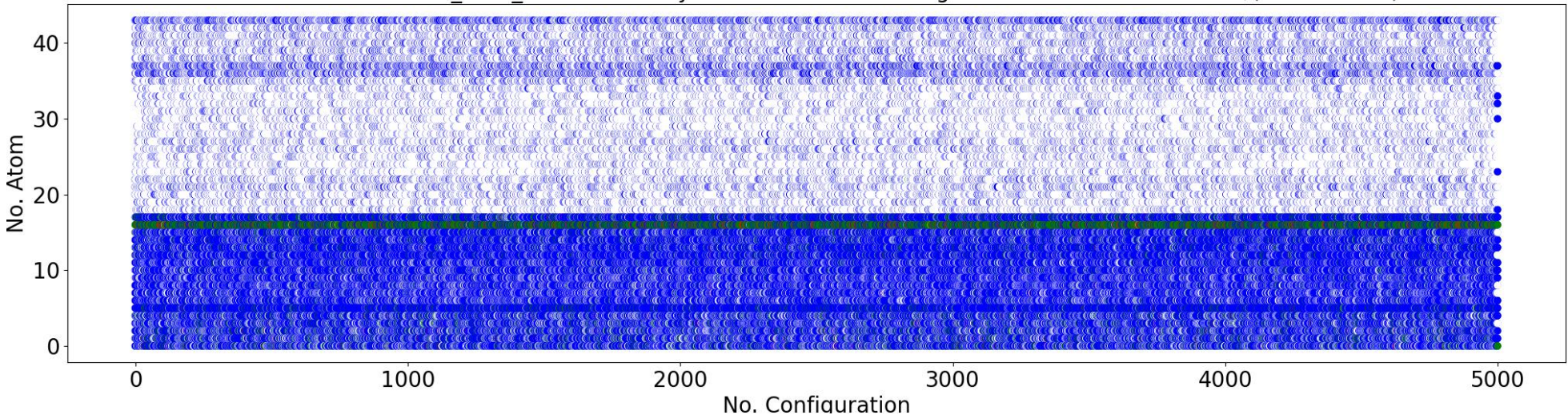




\section{$>50 \mathrm{kcal} /(\mathrm{mol} \cdot \AA)>30 \mathrm{kcal} /(\mathrm{mol} \cdot \AA)>10 \mathrm{kcal} /(\mathrm{mol} \cdot \AA)$}

G7A GAFF2 Force by-atom error 5000 Configs at $600 \mathrm{~K}$ RMSE $31.22 \mathrm{kcal} /\left(\mathrm{mol} * \mathrm{~A}^{*}\right.$ atom)

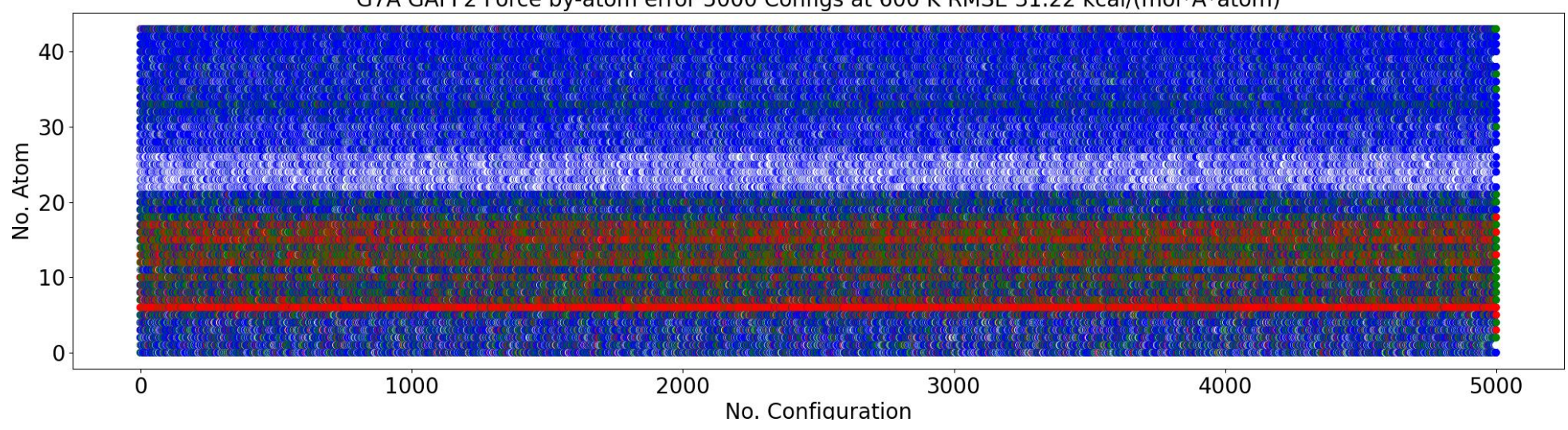

G7A FM-PM6-D3H4X_from_GAFF2 Force by-atom error 5000 Configs at $600 \mathrm{~K}$ RMSE $21.67 \mathrm{kcal} /(\mathrm{mol}$ *A*atom)

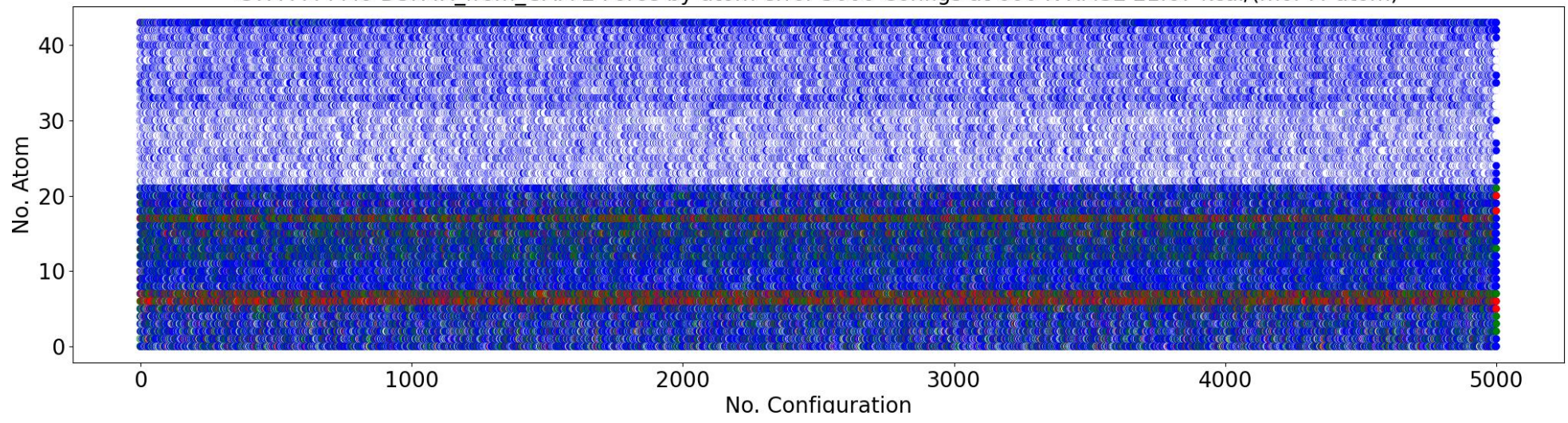

G7A GAFF2 Force by-atom error 5000 Configs at $300 \mathrm{~K}$ RMSE $26.66 \mathrm{kcal} /\left(\mathrm{mol} * \mathrm{~A}^{*}\right.$ atom)

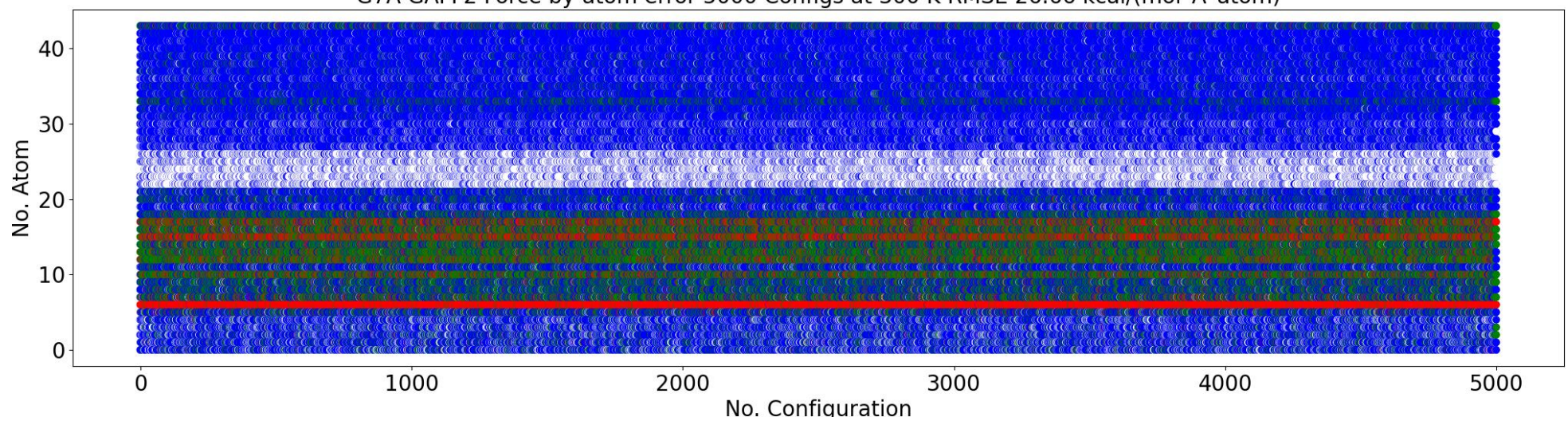

G7A FM-PM6-D3H4X_from_GAFF2 Force by-atom error 5000 Configs at $300 \mathrm{~K}$ RMSE $16.49 \mathrm{kcal} /(\mathrm{mol} * A * a t o m)$

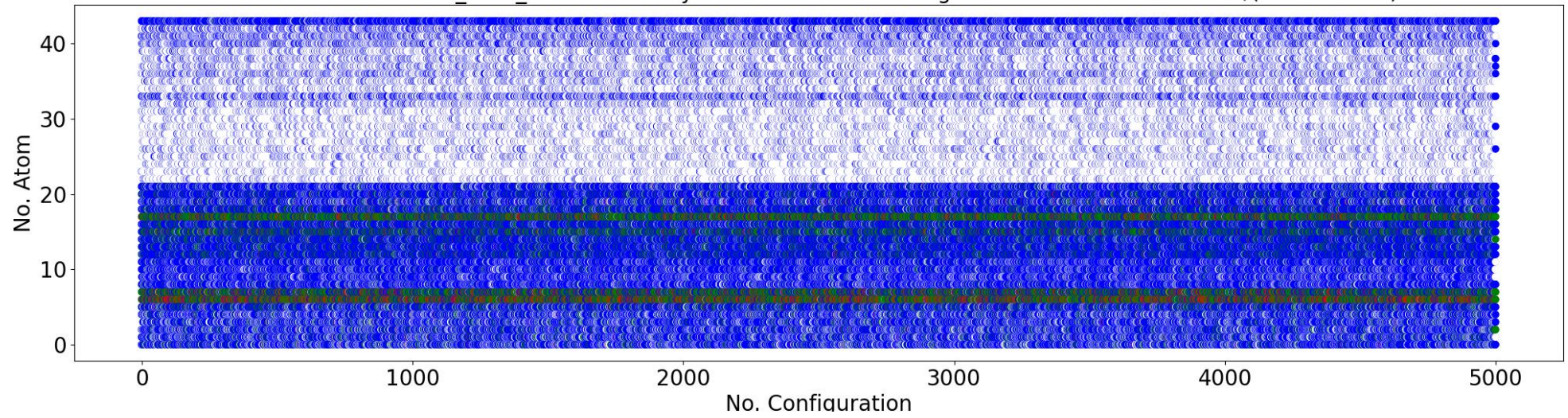


Fig. S3. The correlations between the MM and ab initio QM (BLYP-D4) energetics calculated from $10 \mathrm{~ns}$ trajectories generated at $300 \mathrm{~K}$ in vacuo for the host and 7 guest molecules. The sampling interval is $10 \mathrm{ps}$ and there are 1000 independent configurations in total. The RMSE and MAE of the original parameter set and the newly fitted force-matching set are also presented. Note that the protonated and deprotonated forms of G5 Ketamine are fitted separately.
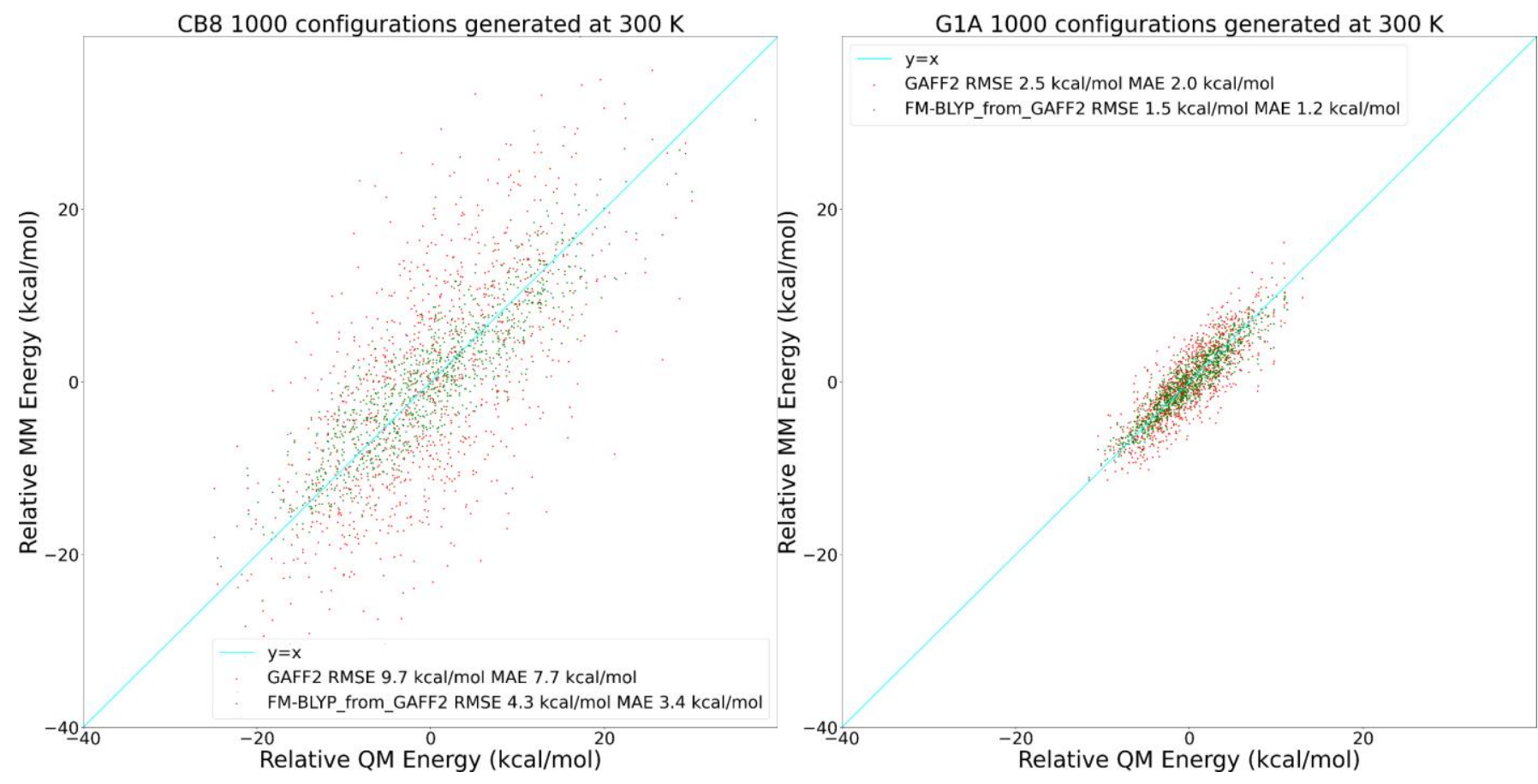

G2A 1000 configurations generated at $300 \mathrm{~K}$
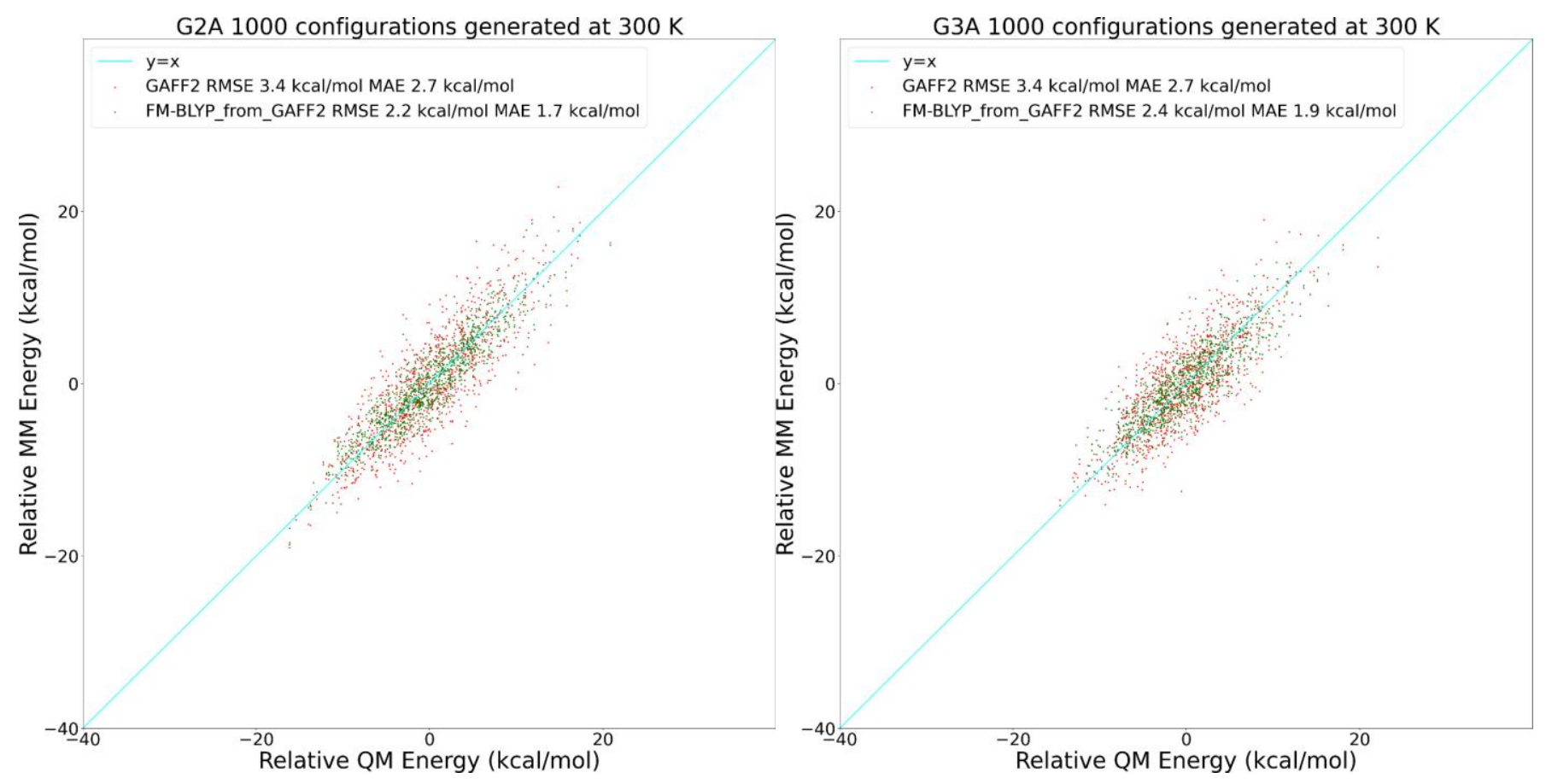

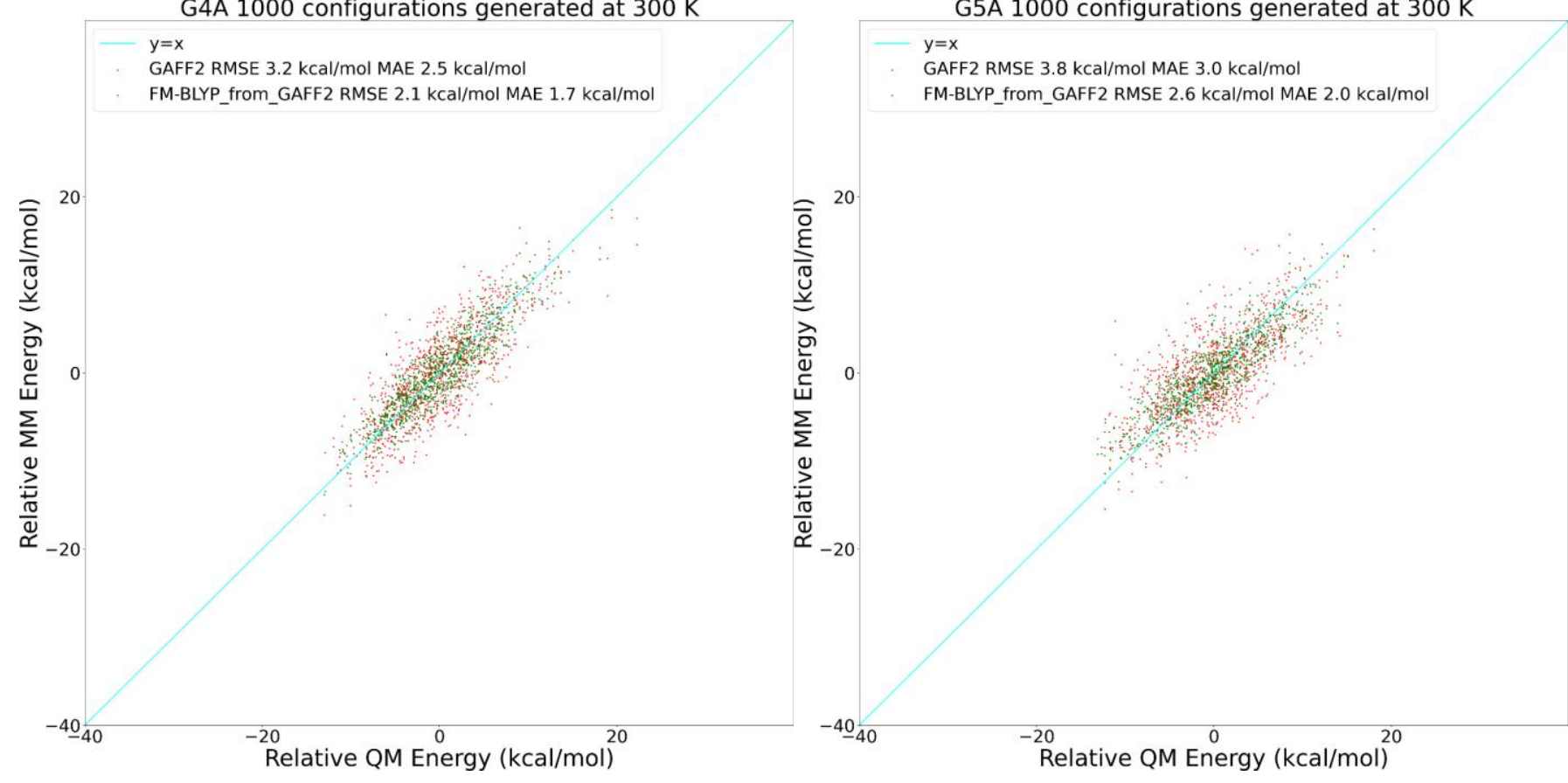

G5B 1000 configurations generated at $300 \mathrm{~K}$

G6A 1000 configurations generated at $300 \mathrm{~K}$
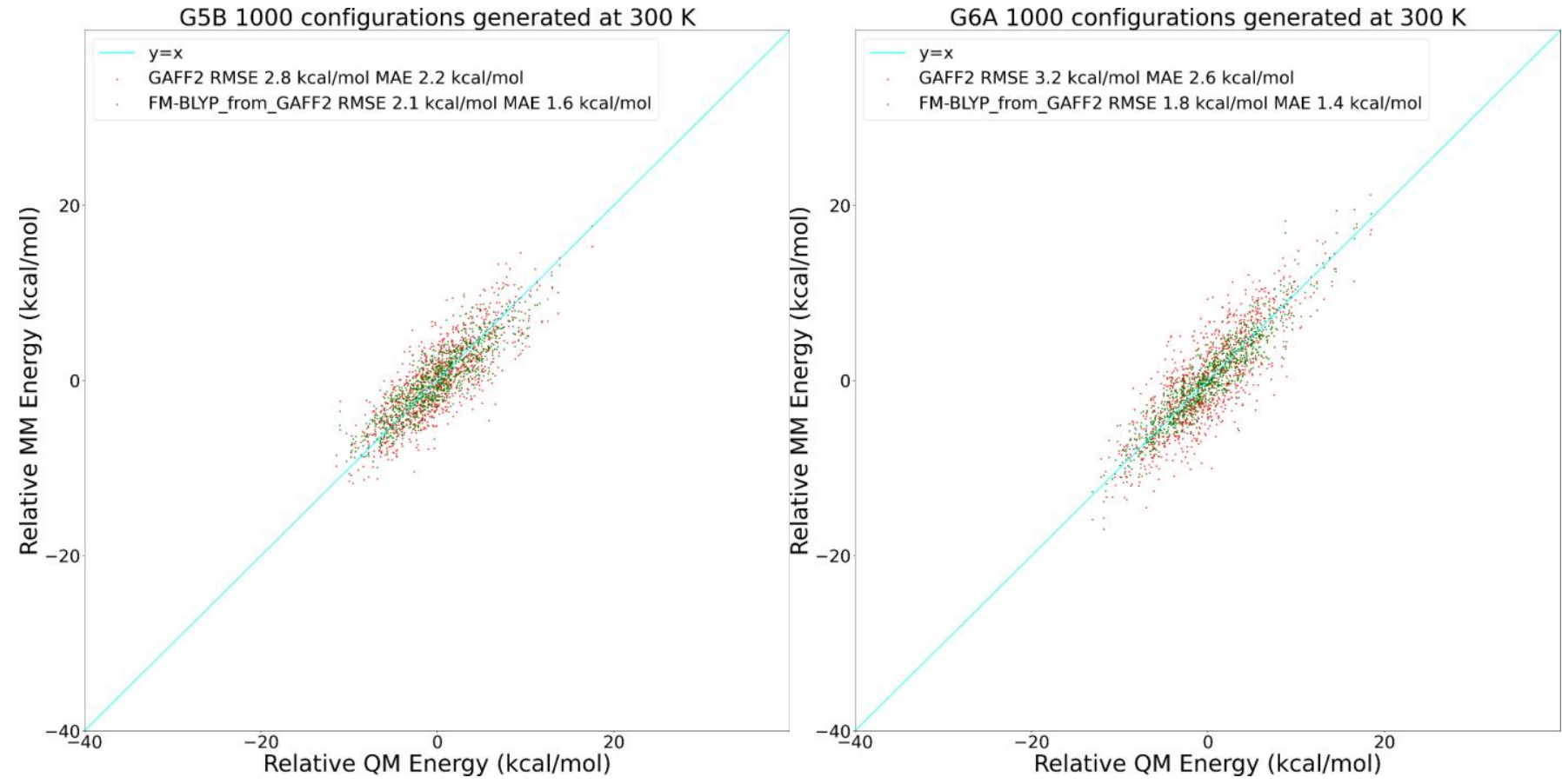
G7A 1000 configurations generated at $300 \mathrm{~K}$

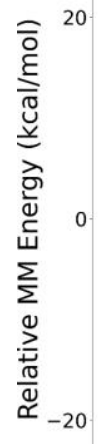

GAFF2 RMSE $4.0 \mathrm{kcal} / \mathrm{mol}$ MAE $3.1 \mathrm{kcal} / \mathrm{mol}$

FM-BLYP_from_GAFF2 RMSE $2.8 \mathrm{kcal} / \mathrm{mol}$ MAE $2.2 \mathrm{kcal} / \mathrm{mol}$

$-40$

$\stackrel{-20}{\text { Relative QM Energy }(\mathrm{kcal} / \mathrm{mol})}$ 
Fig. S4. The time series of the errors of atomic forces $\left(\left\|\Delta \mathbf{F}_{i}\right\|_{2}\right.$ for the $i$ th atom) under the original GAFF2 and the refitted FM-BLYP_from_GAFF2 parameter set calculated from $10 \mathrm{~ns}$ trajectories generated at $300 \mathrm{~K}$ in vacuo for the host and guest molecules. The protonated and deprotonated forms of G5 Ketamine are parameterized and thus tested separately. The sampling interval is $10 \mathrm{ps}$ and there are 1000 independent configurations in total. Red dots for the force errors larger than $50 \mathrm{kcal} /(\mathrm{mol} \cdot \AA)$, green for force errors larger than $30 \mathrm{kcal} /(\mathrm{mol} \cdot \AA)$, blue for errors larger than $10 \mathrm{kcal} /(\mathrm{mol} \cdot \AA)$, and white for the other small-error points. The overall RMSEs of atomic forces of each molecule in $\mathrm{kcal} /(\mathrm{mol} \cdot \AA \cdot$ atom $)$ under the original GAFF2 and the newly obtained FM-BLYP parameter sets are also given.

\section{$>50 \mathrm{kcal} /(\mathrm{mol} \cdot \AA)>30 \mathrm{kcal} /(\mathrm{mol} \cdot \AA)>10 \mathrm{kcal} /(\mathrm{mol} \cdot \AA)$}
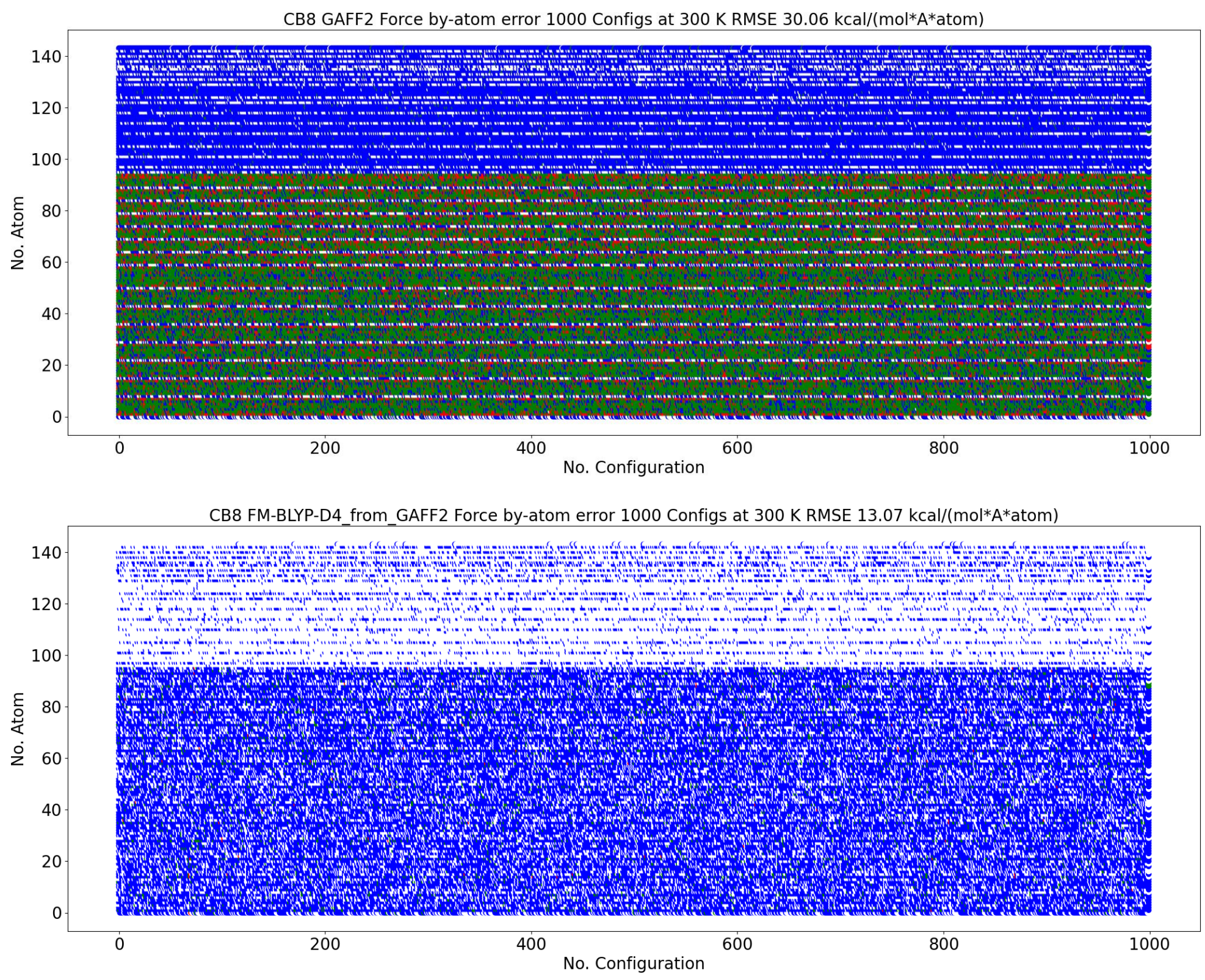


\section{$>50 \mathrm{kcal} /(\mathrm{mol} \cdot \AA)>30 \mathrm{kcal} /(\mathrm{mol} \cdot \AA)>10 \mathrm{kcal} /(\mathrm{mol} \cdot \AA)$}

G1A GAFF2 Force by-atom error 1000 Configs at $300 \mathrm{~K}$ RMSE $15.81 \mathrm{kcal} /(\mathrm{mol} * \mathrm{~A} *$ atom)

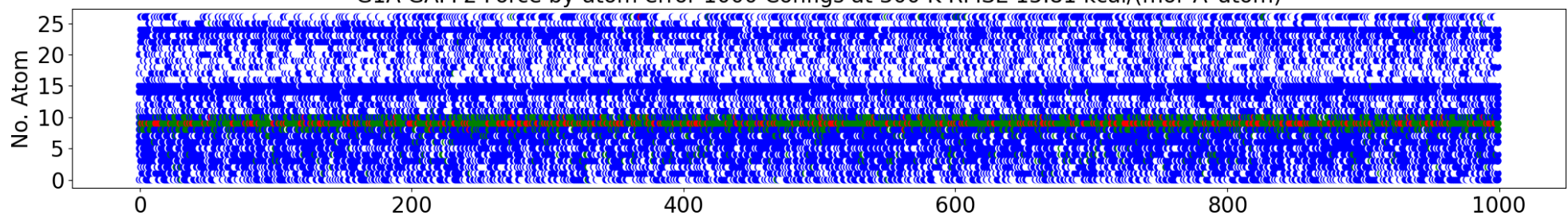

G1A FM-BLYP-D4_from_GAFF2 Force by-atom error 1000 Configs at $300 \mathrm{~K} \mathrm{RMSE} 9.53 \mathrm{kcal} /(\mathrm{mol} * \mathrm{~A} *$ atom)

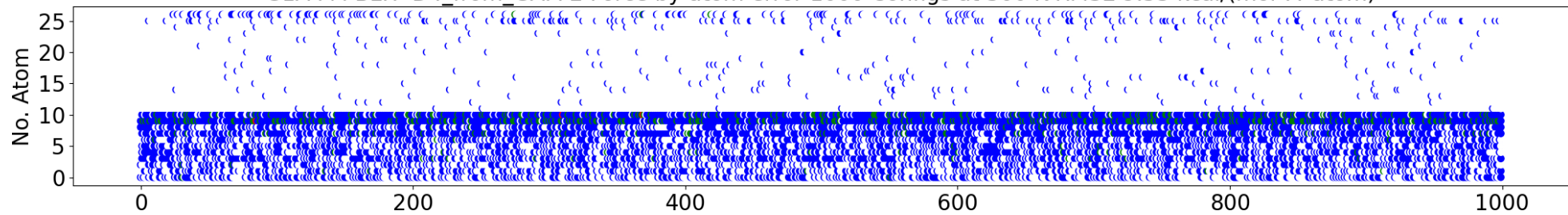

G2A GAFF2 Force by-atom error 1000 Configs at $300 \mathrm{~K}$ RMSE $16.21 \mathrm{kcal} /\left(\mathrm{mol} * \mathrm{~A}^{*}\right.$ atom)

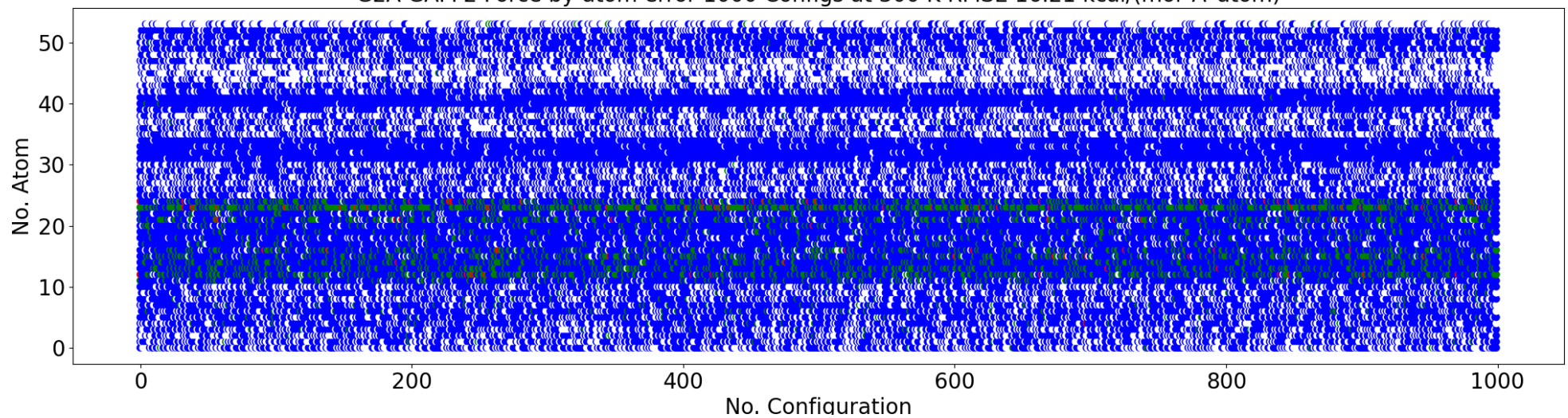

G2A FM-BLYP_from_GAFF2 Force by-atom error 1000 Configs at $300 \mathrm{~K}$ RMSE $9.35 \mathrm{kcal} /(\mathrm{mol} * \mathrm{~A} *$ atom)

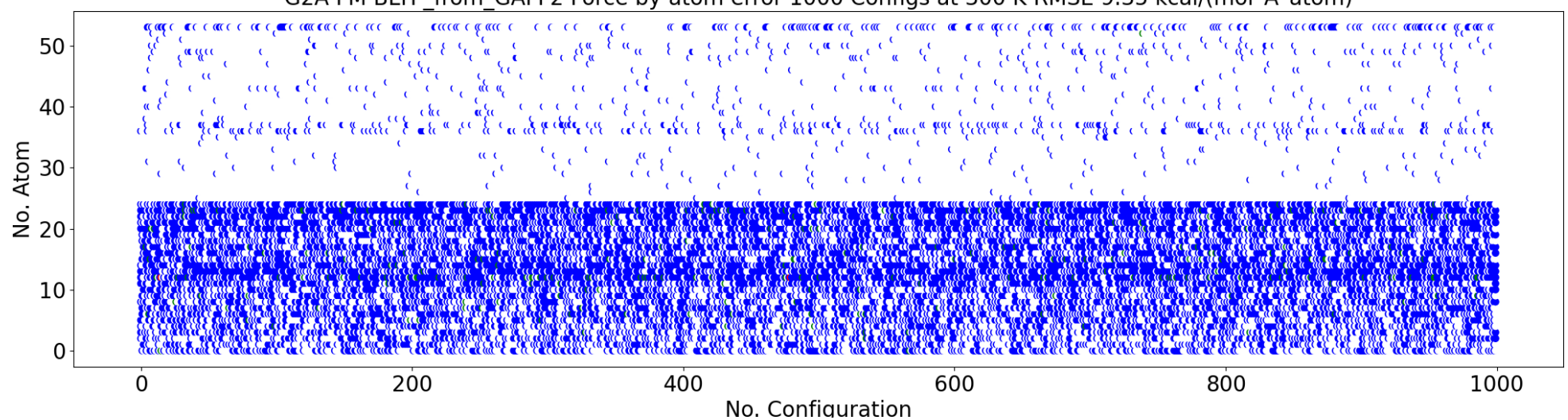




\section{$>50 \mathrm{kcal} /(\mathrm{mol} \cdot \AA)>30 \mathrm{kcal} /(\mathrm{mol} \cdot \AA)>10 \mathrm{kcal} /(\mathrm{mol} \cdot \AA)$}

G3A GAFF2 Force by-atom error 1000 Configs at $300 \mathrm{~K}$ RMSE $18.37 \mathrm{kcal} /\left(\mathrm{mol} * \mathrm{~A}^{*}\right.$ atom)

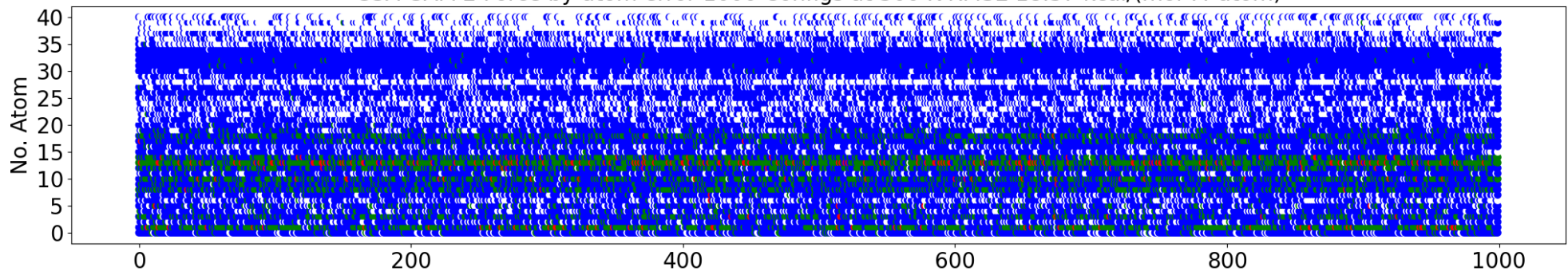

G3A FM-BLYP-D4_from_GAFF2 Force by-atom error 1000 Configs at $300 \mathrm{~K}$ RMSE $11.47 \mathrm{kcal} /(\mathrm{mol} * \mathrm{~A} *$ atom)

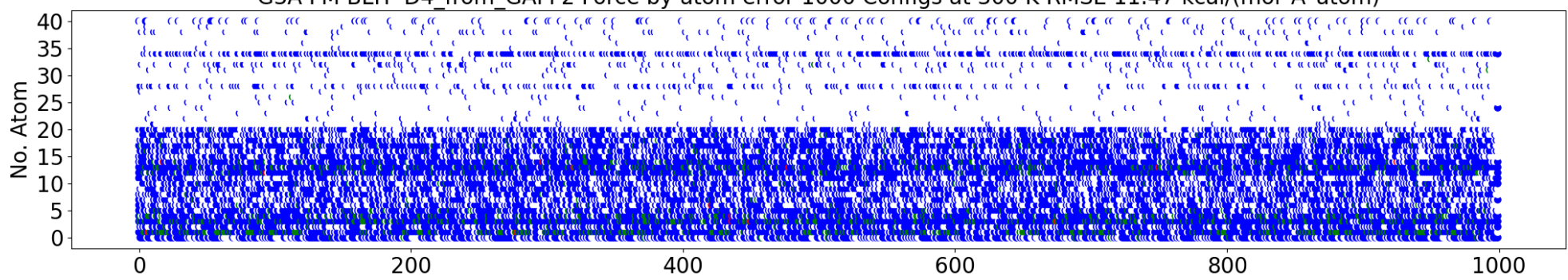

G4A GAFF2 Force by-atom error 1000 Configs at $300 \mathrm{~K}$ RMSE $17.83 \mathrm{kcal} /\left(\mathrm{mol} * \mathrm{~A}^{*}\right.$ atom)

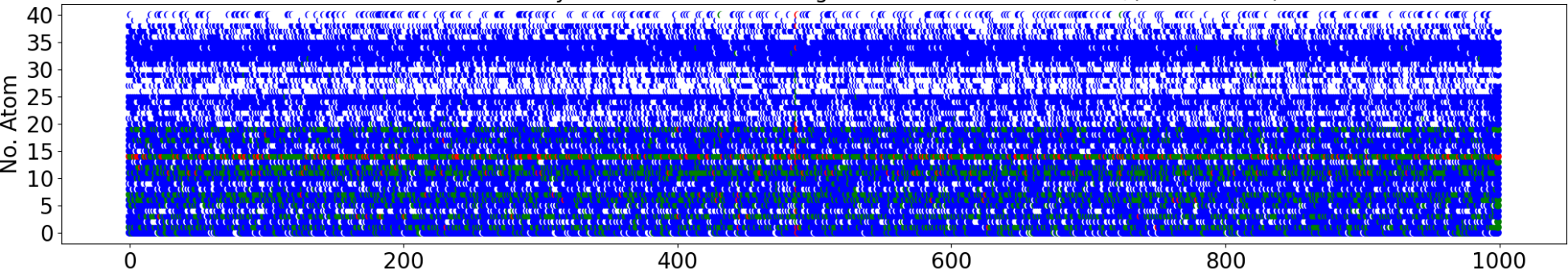

G4A FM-BLYP-D4_from_GAFF2 Force by-atom error 1000 Configs at $300 \mathrm{~K}$ RMSE $11.29 \mathrm{kcal} /(\mathrm{mol} * \mathrm{~A} *$ atom)

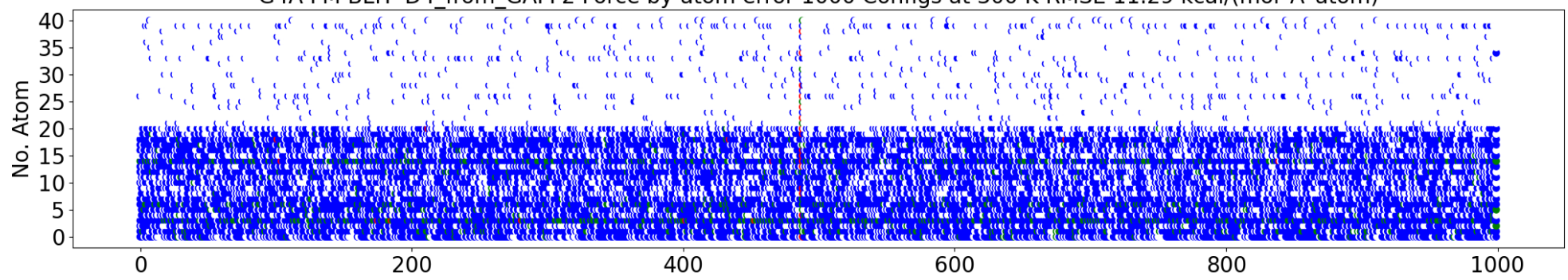




\section{$>50 \mathrm{kcal} /(\mathrm{mol} \cdot \AA)>30 \mathrm{kcal} /(\mathrm{mol} \cdot \AA)>10 \mathrm{kcal} /(\mathrm{mol} \cdot \AA)$}

G5A GAFF2 Force by-atom error 1000 Configs at $300 \mathrm{~K} \mathrm{RMSE} 16.58 \mathrm{kcal} /(\mathrm{mol} * \mathrm{~A}$ *atom)

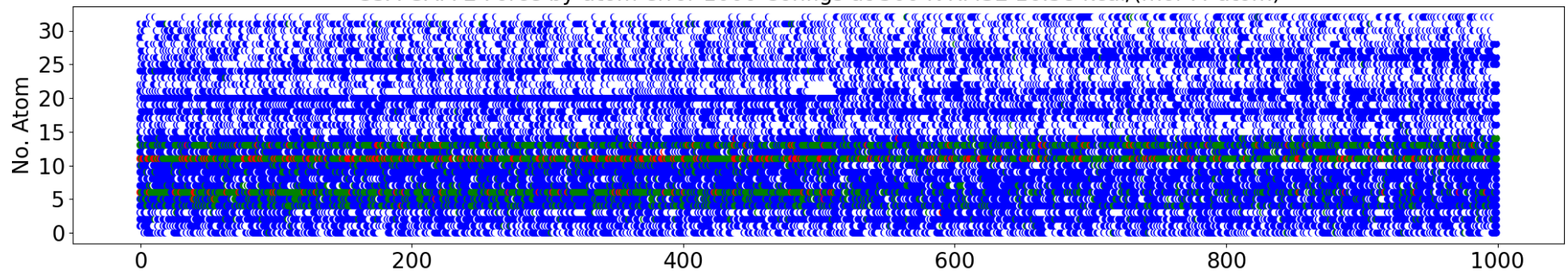

G5A FM-BLYP-D4_from_GAFF2 Force by-atom error 1000 Configs at $300 \mathrm{~K}$ RMSE $11.82 \mathrm{kcal} /$ (mol*A*atom)

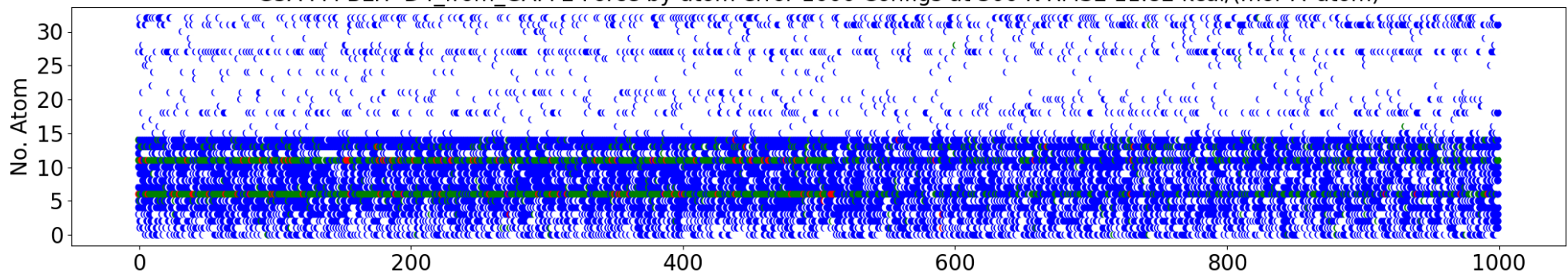

G5B GAFF2 Force by-atom error 1000 Configs at $300 \mathrm{~K}$ RMSE $16.68 \mathrm{kcal} /\left(\mathrm{mol} * A^{*}\right.$ atom)

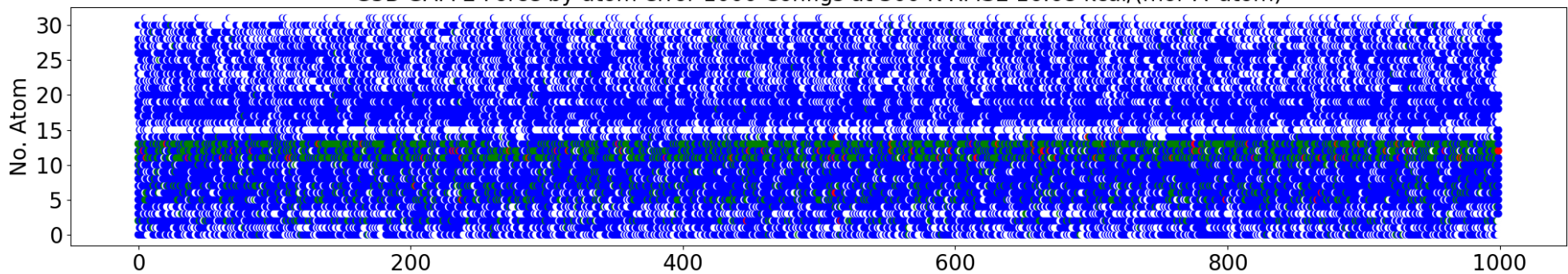

G5B FM-BLYP-D4_from_GAFF2 Force by-atom error 1000 Configs at $300 \mathrm{~K}$ RMSE $10.63 \mathrm{kcal} /$ (mol*A*atom)

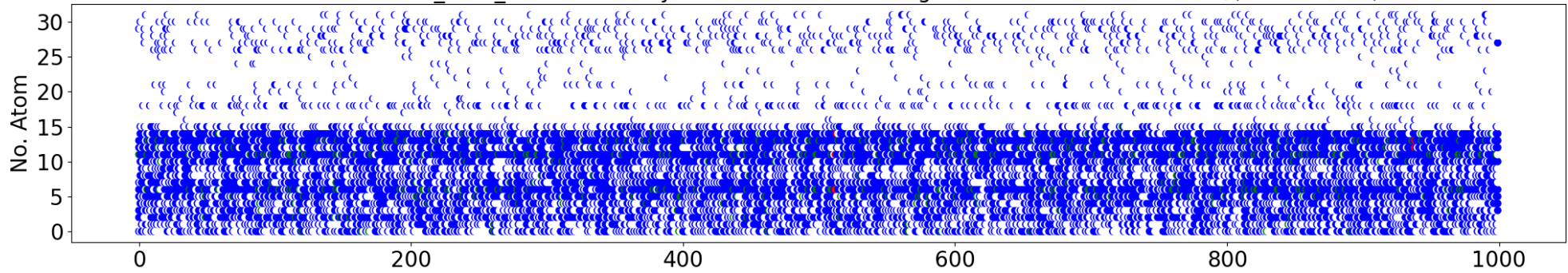




\section{$>50 \mathrm{kcal} /(\mathrm{mol} \cdot \AA)>30 \mathrm{kcal} /(\mathrm{mol} \cdot \AA)>10 \mathrm{kcal} /(\mathrm{mol} \cdot \AA)$}

G6A GAFF2 Force by-atom error 1000 Configs at $300 \mathrm{~K}$ RMSE $16.67 \mathrm{kcal} /\left(\mathrm{mol} * \mathrm{~A}^{*}\right.$ atom)

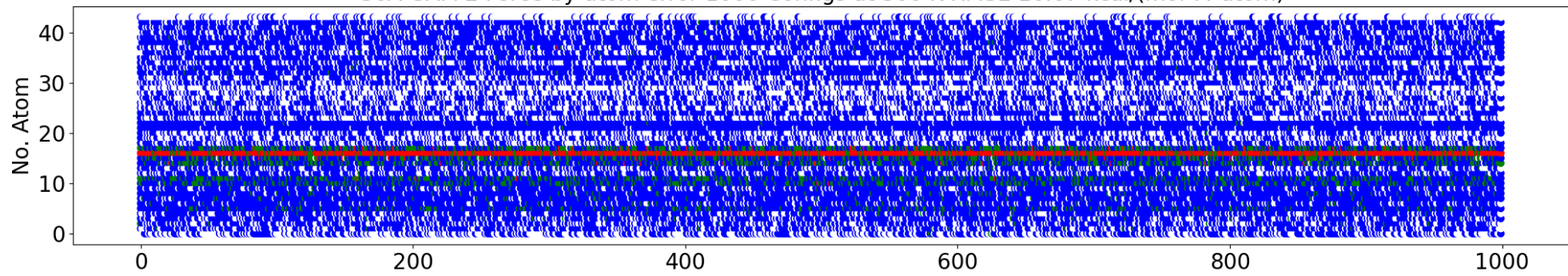

G6A FM-BLYP-D4_from_GAFF2 Force by-atom error 1000 Configs at $300 \mathrm{~K}$ RMSE $9.25 \mathrm{kcal} /(\mathrm{mol} *$ A*atom)

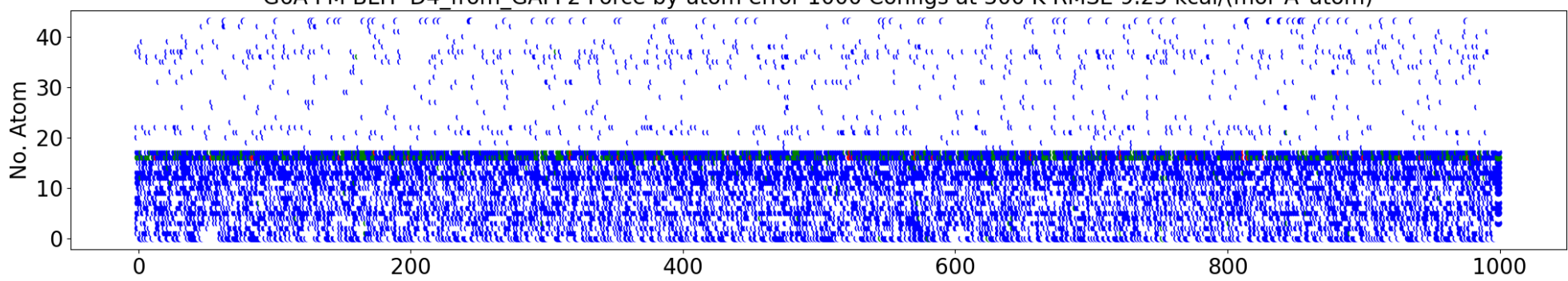

G7A GAFF2 Force by-atom error 1000 Configs at $300 \mathrm{~K}$ RMSE $17.98 \mathrm{kcal} /\left(\mathrm{mol} * \mathrm{~A}^{*}\right.$ atom)

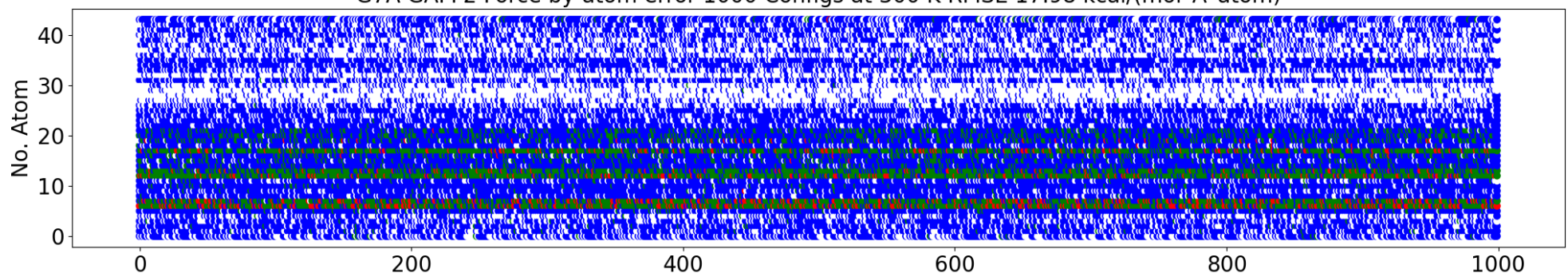

G7A FM-BLYP-D4_from_GAFF2 Force by-atom error 1000 Configs at $300 \mathrm{~K}$ RMSE $12.46 \mathrm{kcal} /(\mathrm{mol} * \mathrm{~A} *$ atom)

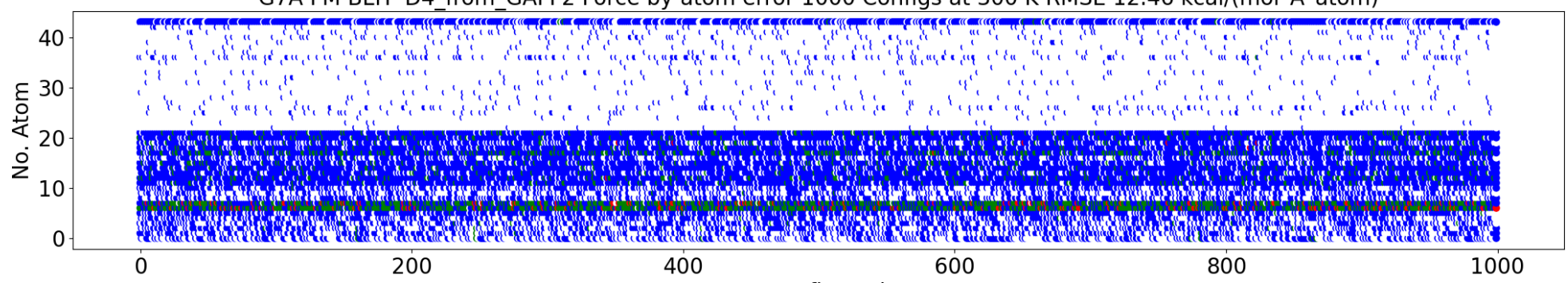


Fig. S5. Dihedral terms defined in GAFF derivatives describing the intra-molecular conformational preference of the host CB8.
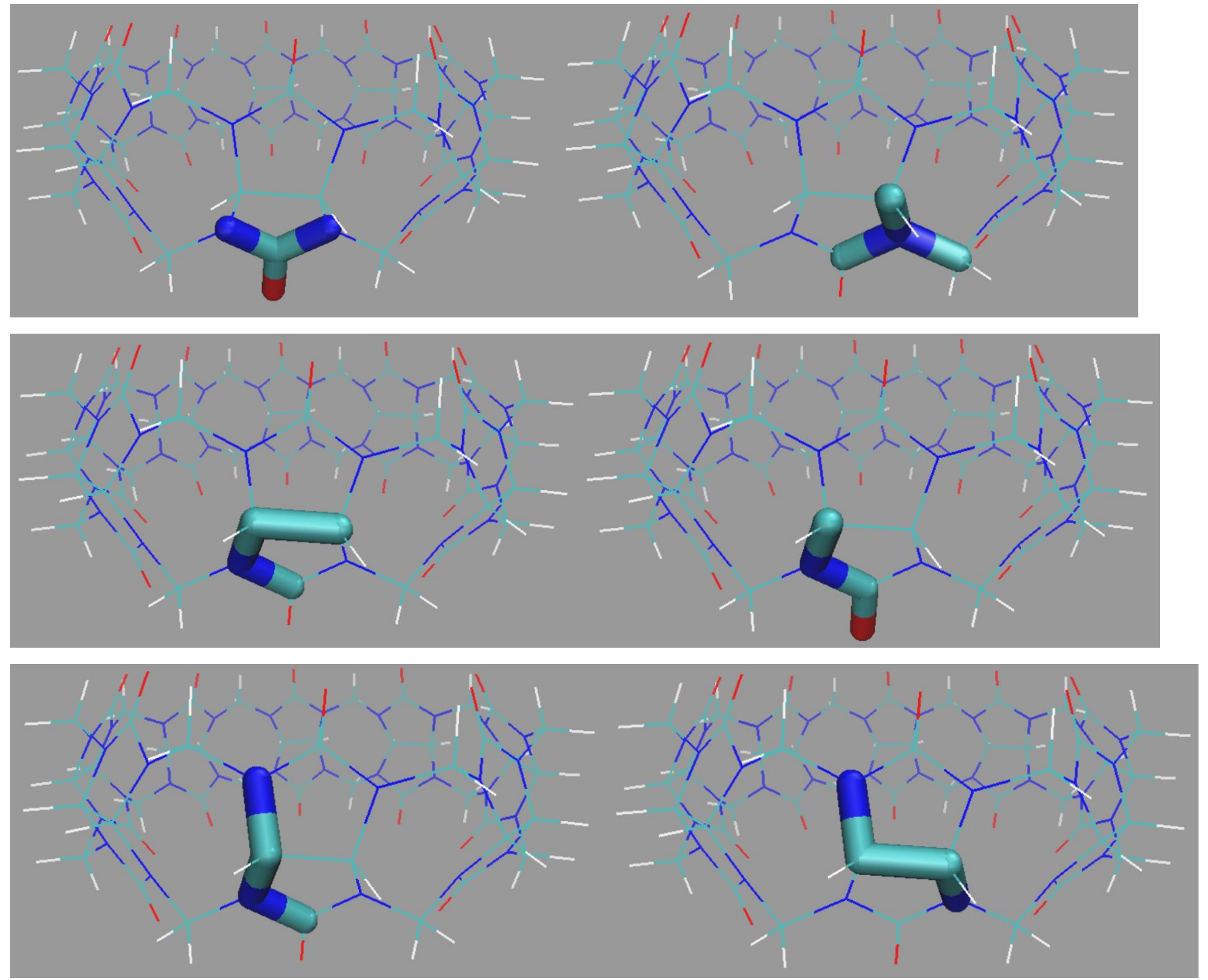
Fig. S6. The number of contacts between all atoms of the host CB8 and the guests and the by-host-atom decomposition during metadynamics simulations under the GAFF2 force field. The y-axis represents the serial number of host atom. Among the 144 atoms of the host CB8, the first 96 atoms are heavy atoms, and the other 48 atoms are hydrogen atoms. All atoms of the host and the guest are included in the calculation. Red dots denote contacts larger than 10, green dots represent contact number between 5 and 10, blue ones are those larger than 1, and the other are represented by white dots.
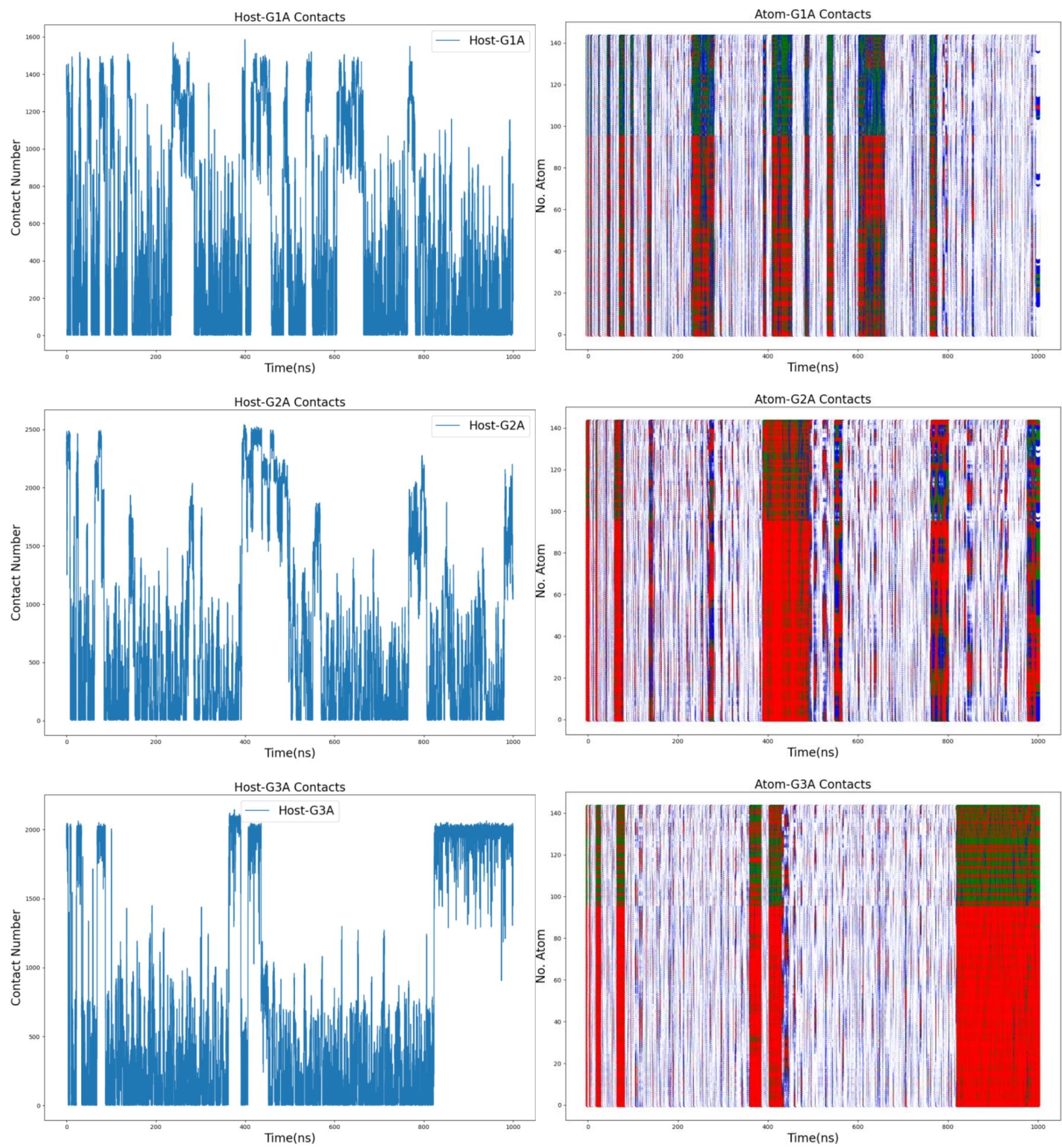

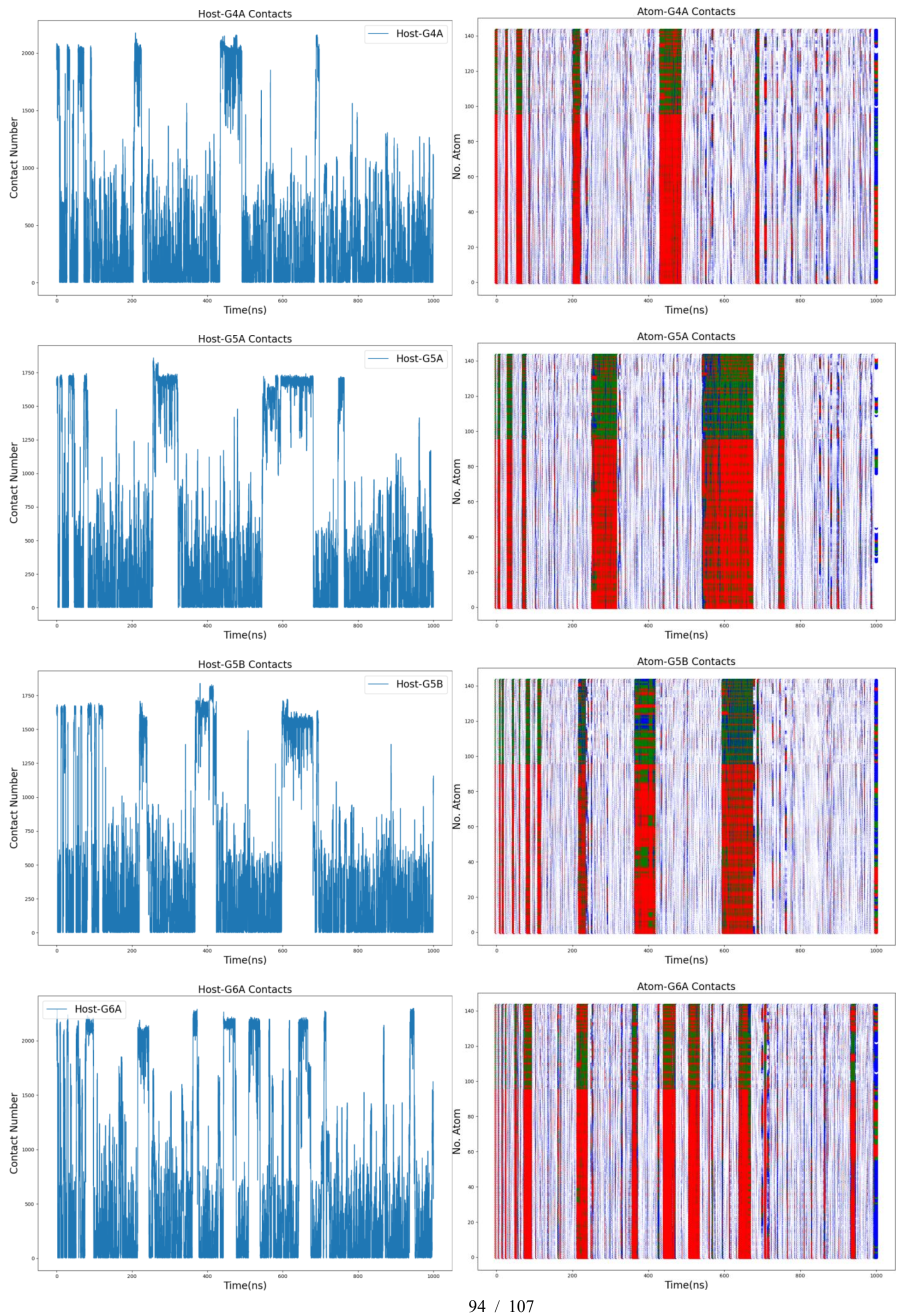

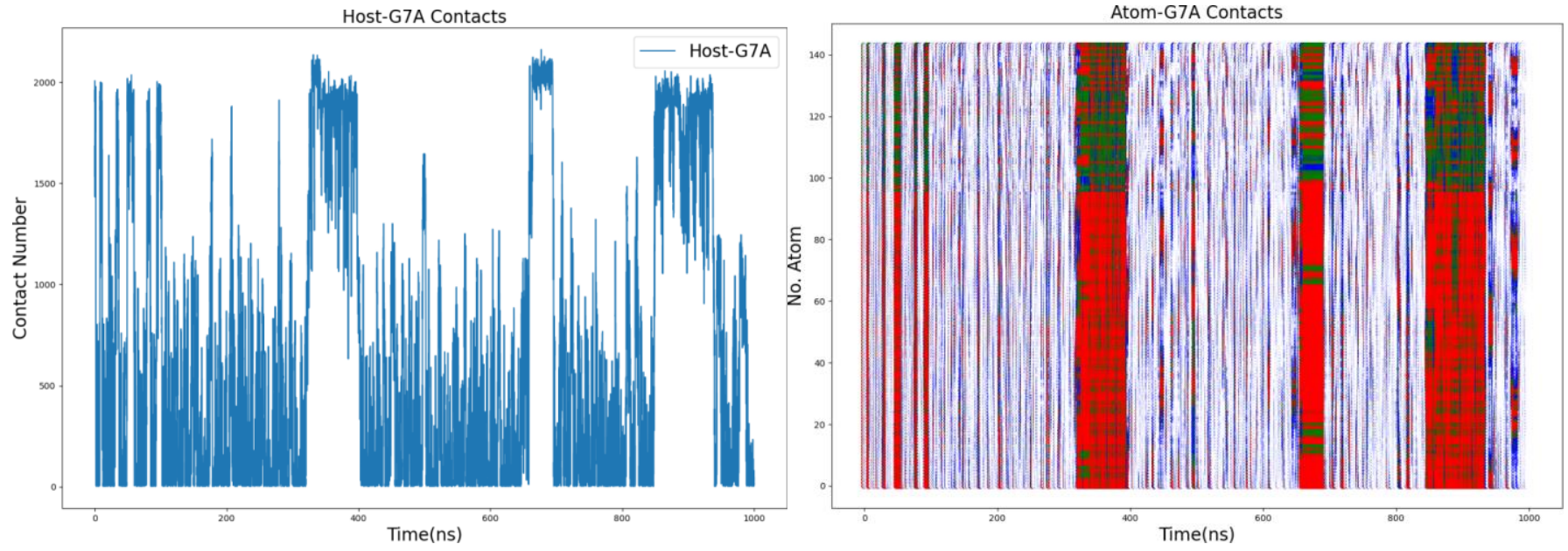
Fig. S7. The number of contacts between all atoms of the host CB8 and the guests and the by-host-atom decomposition during metadynamics simulations with the FM-PM6 parameter set. The y-axis represents the serial number of host atom. Among the 144 atoms of the host CB8, the first 96 atoms are heavy atoms, and the other 48 atoms are hydrogen atoms. All atoms of the host and the guest are included in the calculation. Red dots denote contacts larger than 10, green dots represent contact number between 5 and 10, blue ones are those larger than 1, and the other are represented by white dots.
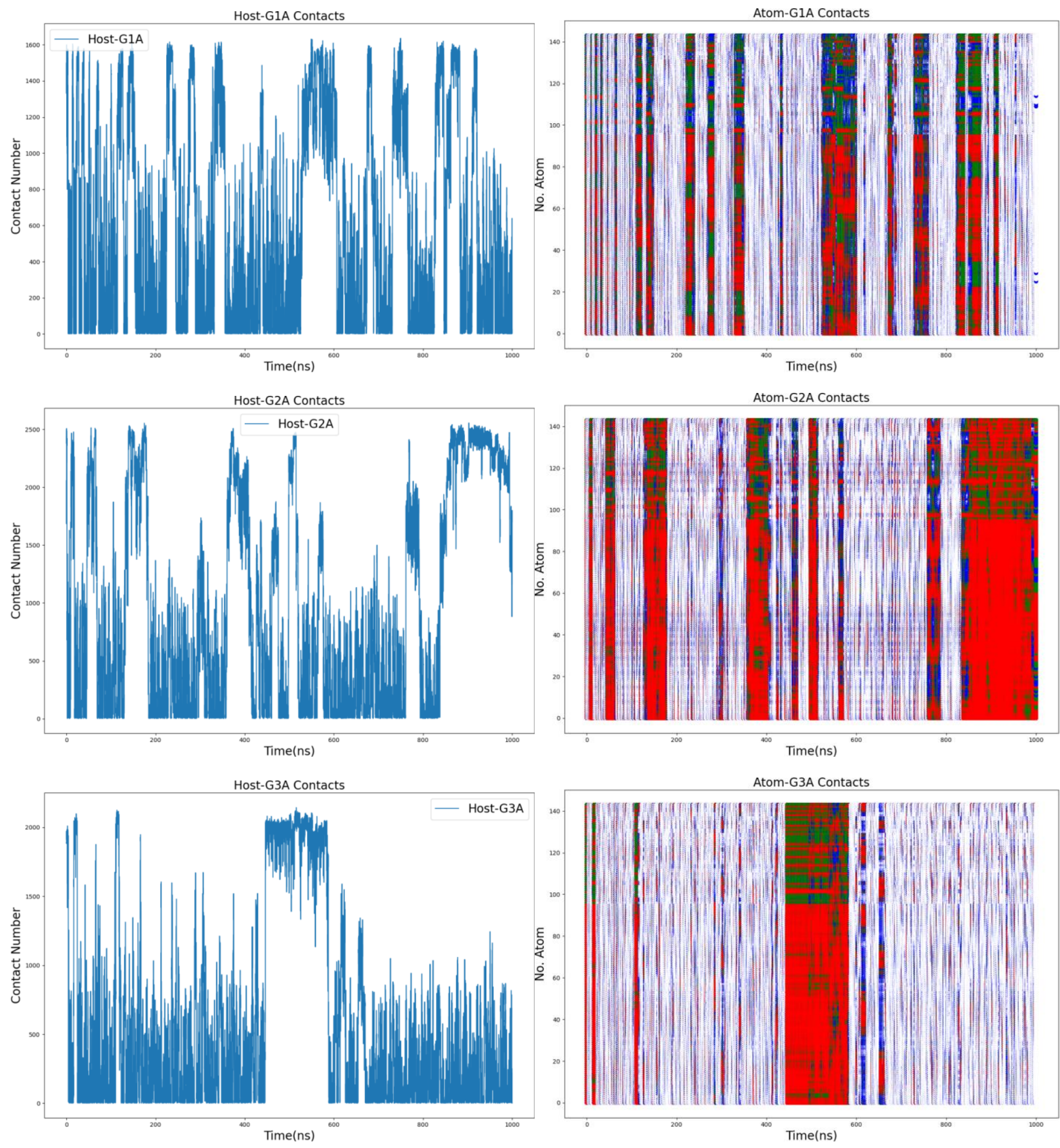

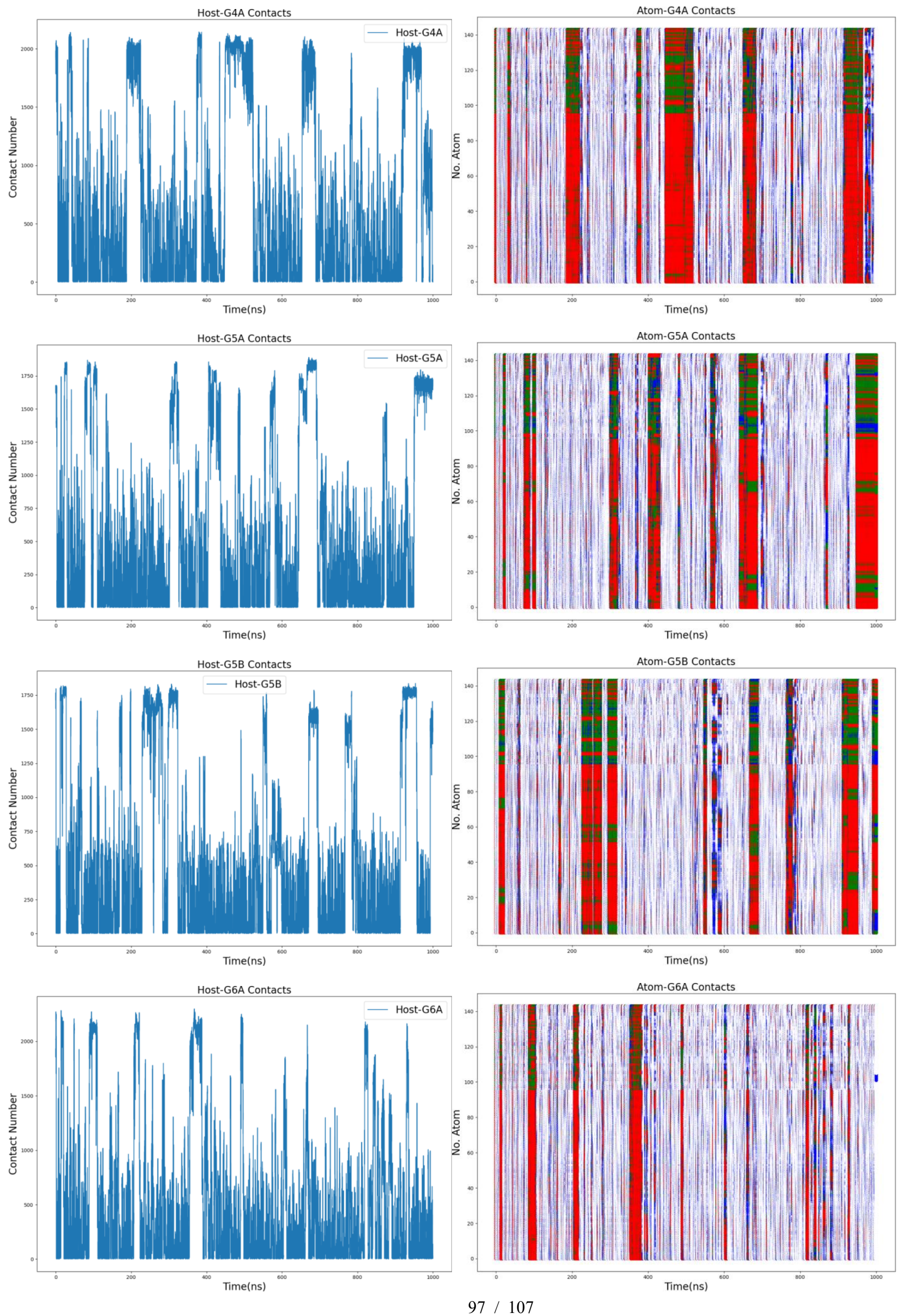
Host-G7A Contacts

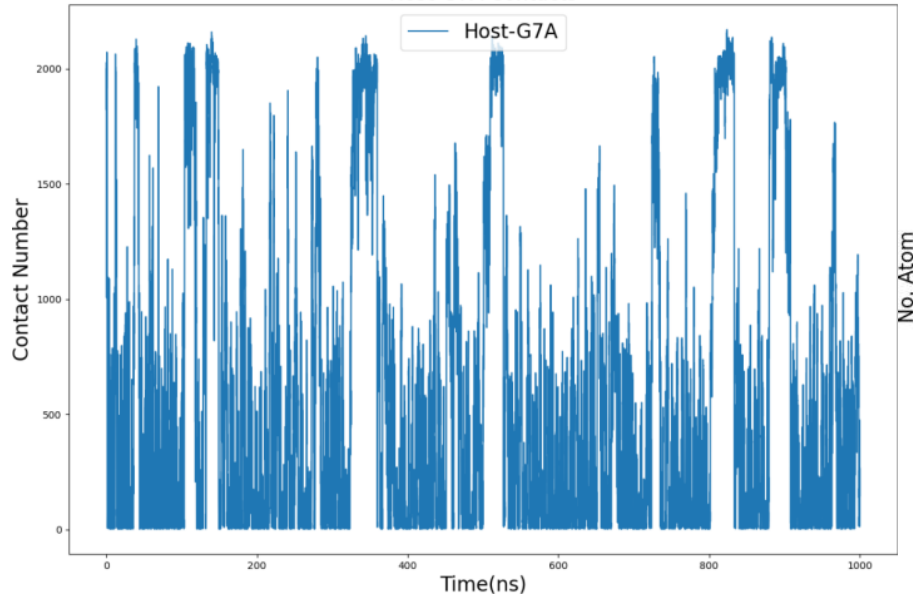

Atom-G7A Contacts

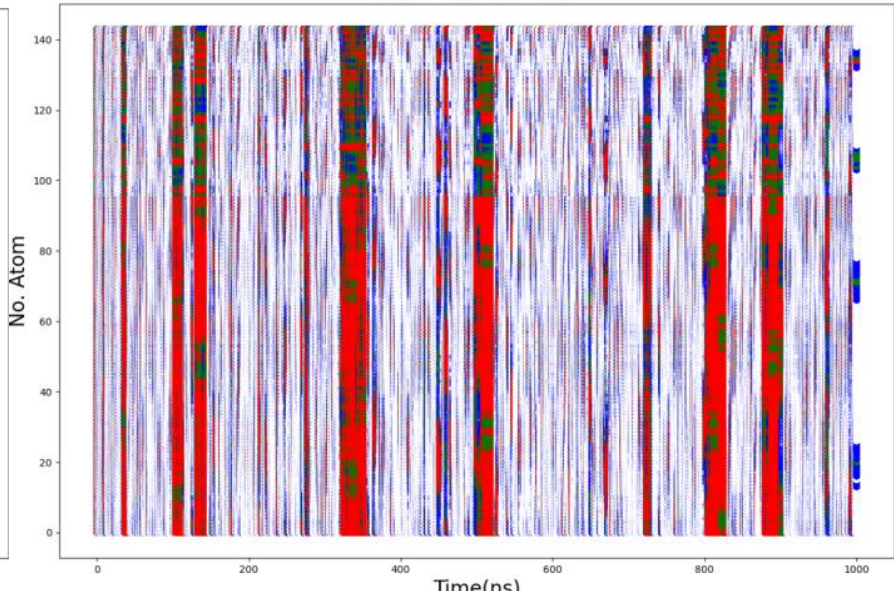


Fig. S8. The number of contacts between all atoms of the host CB8 and the guests and the by-host-atom decomposition during metadynamics simulations with the FM-BLYP parameter set. The y-axis represents the serial number of host atom. Among the 144 atoms of the host CB8, the first 96 atoms are heavy atoms, and the other 48 atoms are hydrogen atoms. All atoms of the host and the guest are included in the calculation. Red dots denote contacts larger than 10, green dots represent contact number between 5 and 10, blue ones are those larger than 1, and the other are represented by white dots.
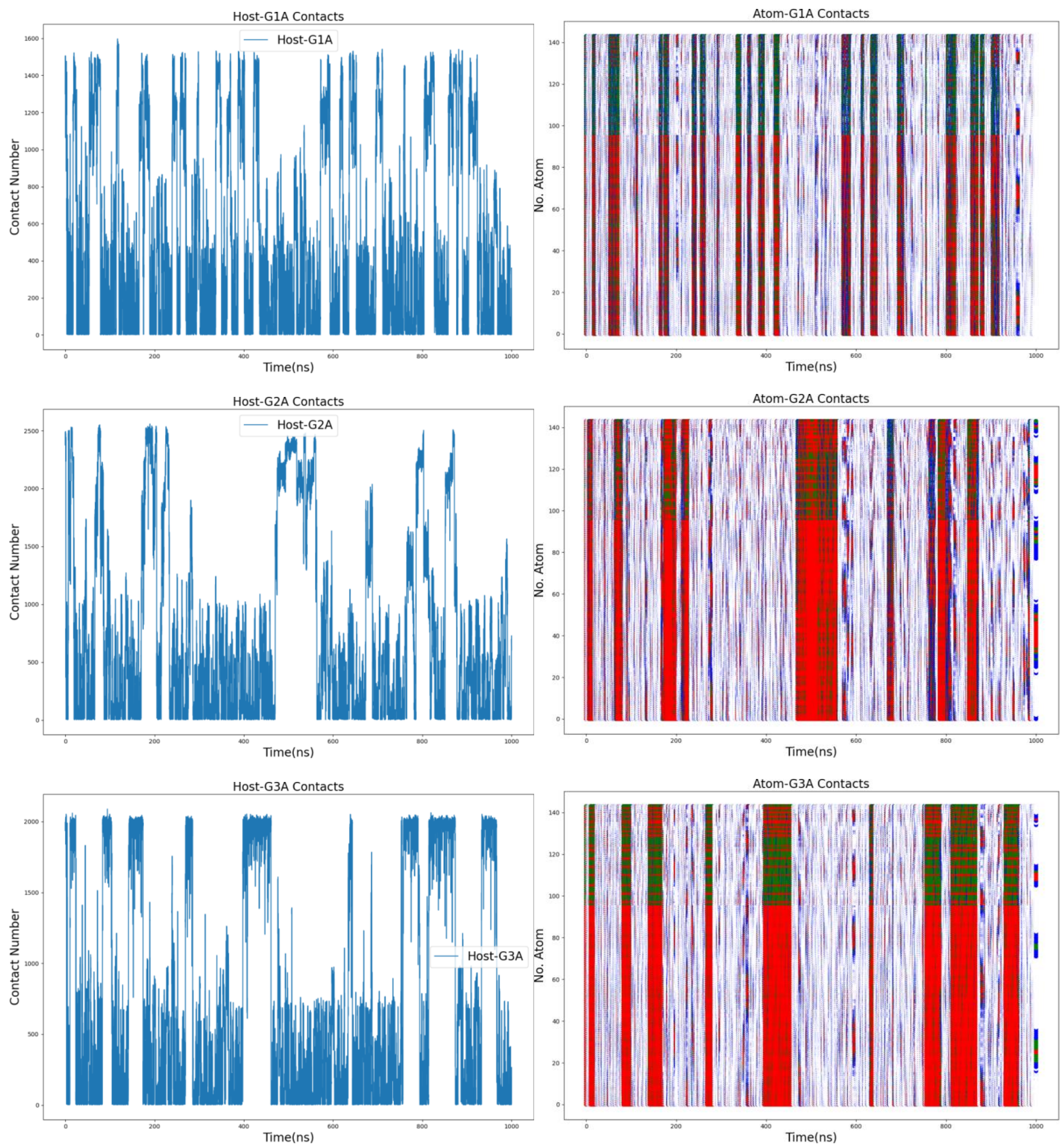

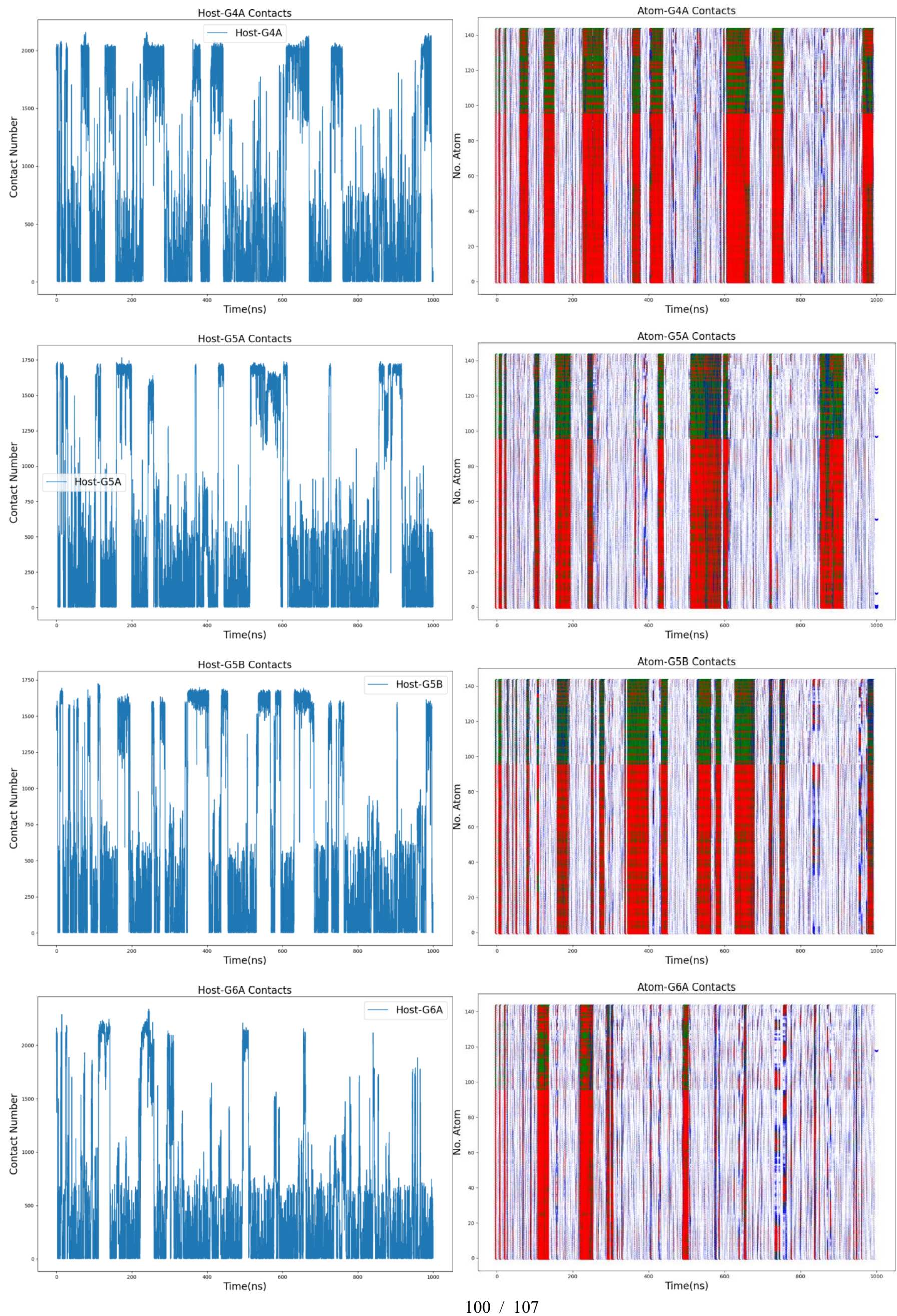
Host-G7A Contacts

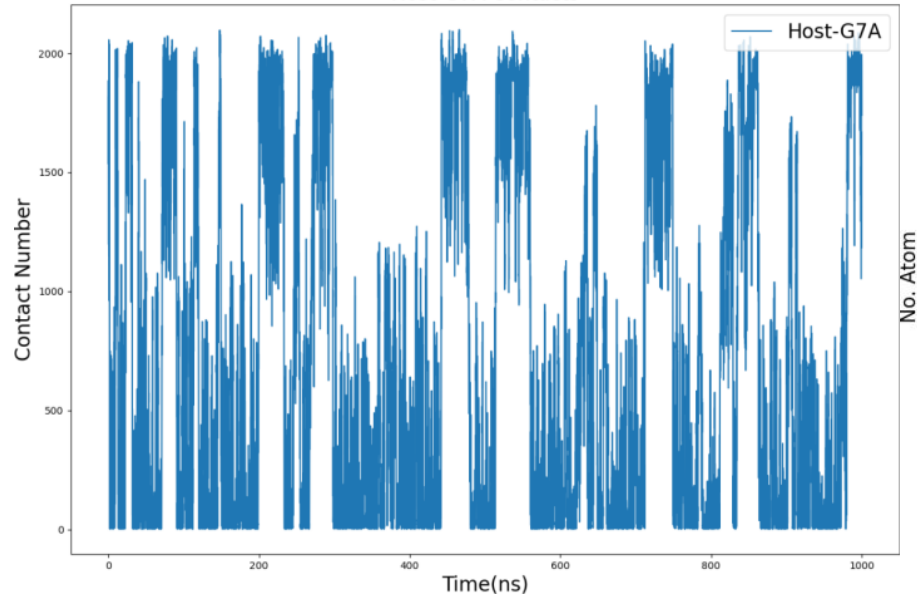

Atom-G7A Contacts

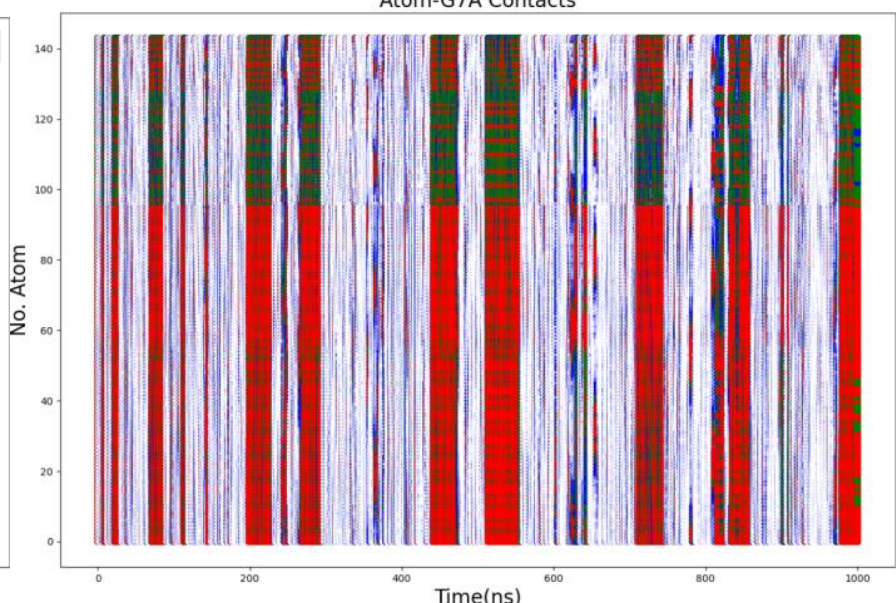


Fig. S9. 2D $\rho-C$ free energy surfaces in $\mathrm{kcal} / \mathrm{mol}$ with the GAFF2 force field. Representative structures extracted from the global minimum and the other low-energy local minima are also shown. A worth noting difference between the current GAFF2 binding poses and the GAFF ones reported in our previous work is the closeness or the degree of distortion of the host cavity. As has been shown in the current work, the previously employed GAFF parameter set provides more flexible/softer host ring, which makes it squashed significantly in the bound state. However, the current GAFF2 parameter set describes a stiffer host ring, which is less distorted when coordinating the guest molecules.
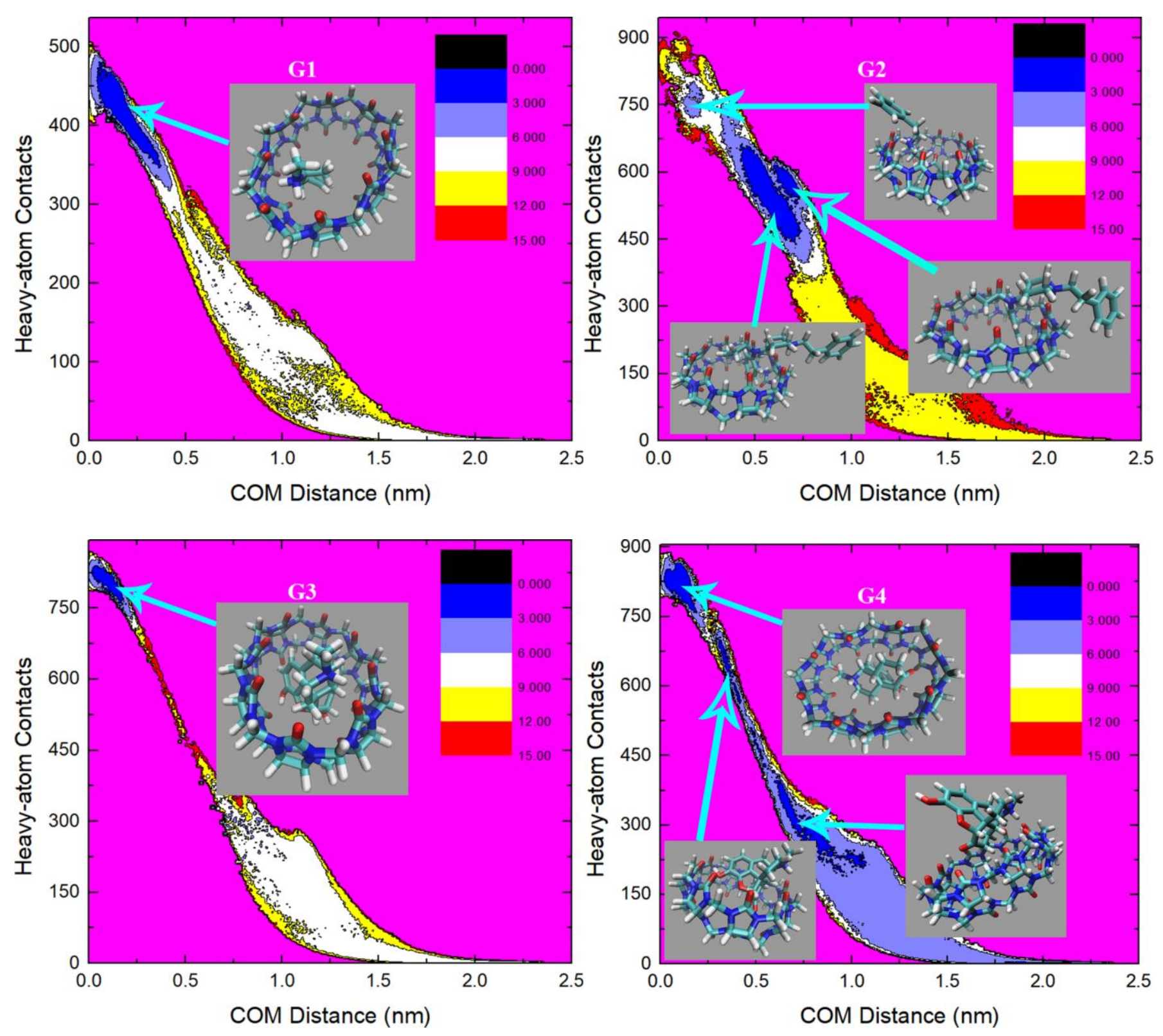

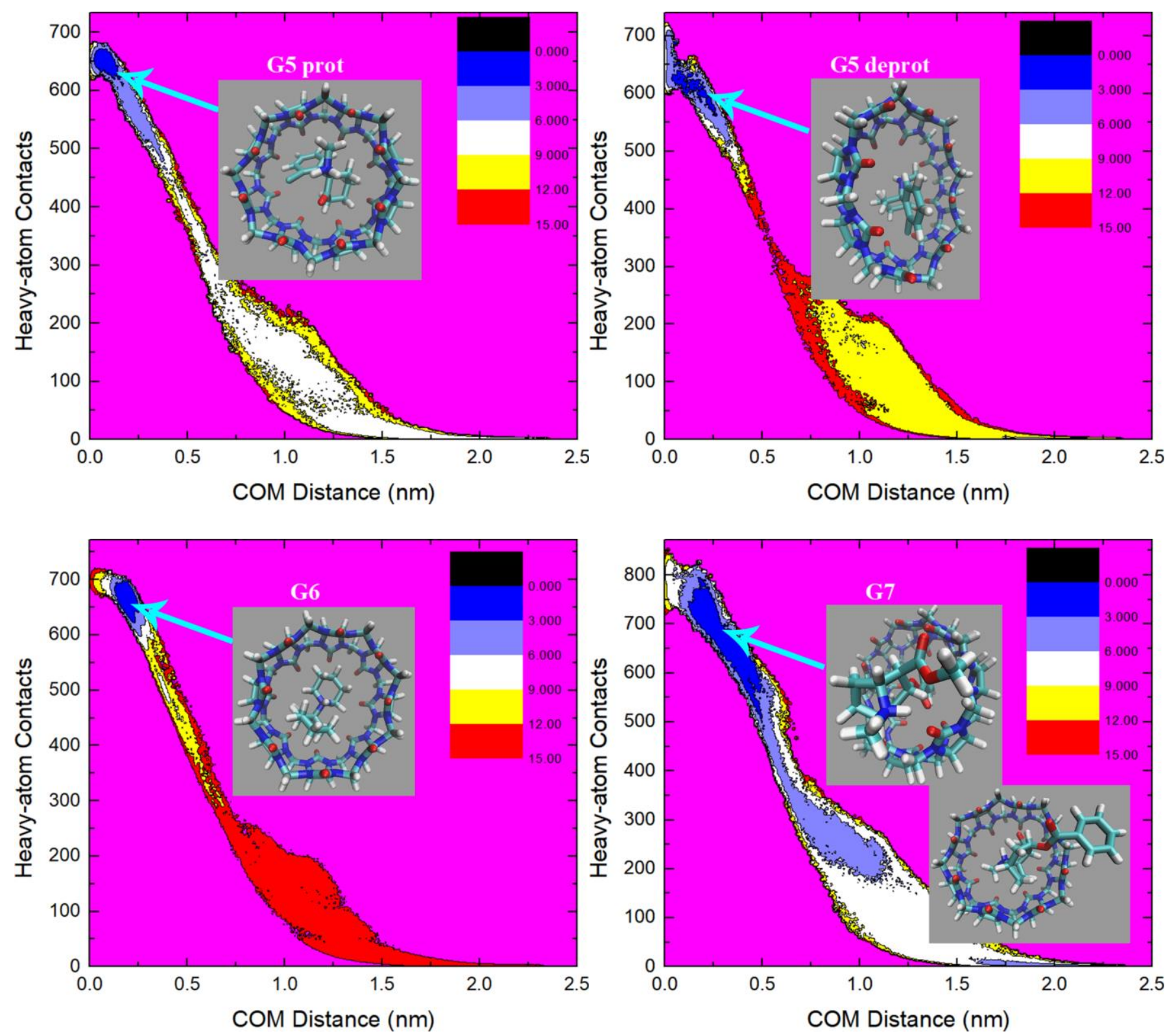
Fig. S10. 2D $\rho-C$ free energy surfaces in $\mathrm{kcal} / \mathrm{mol}$ obtained with the FM-PM6 parameter set. Representative structures extracted from the global minimum and the other low-energy local minima are also shown. Compared with the previous GAFF2 binding modes, those observed under FM-PM6 generally have more flexible and irregularly shaped host cavities, which is in agreement with the observations in unbiased simulations reported in the previous section.
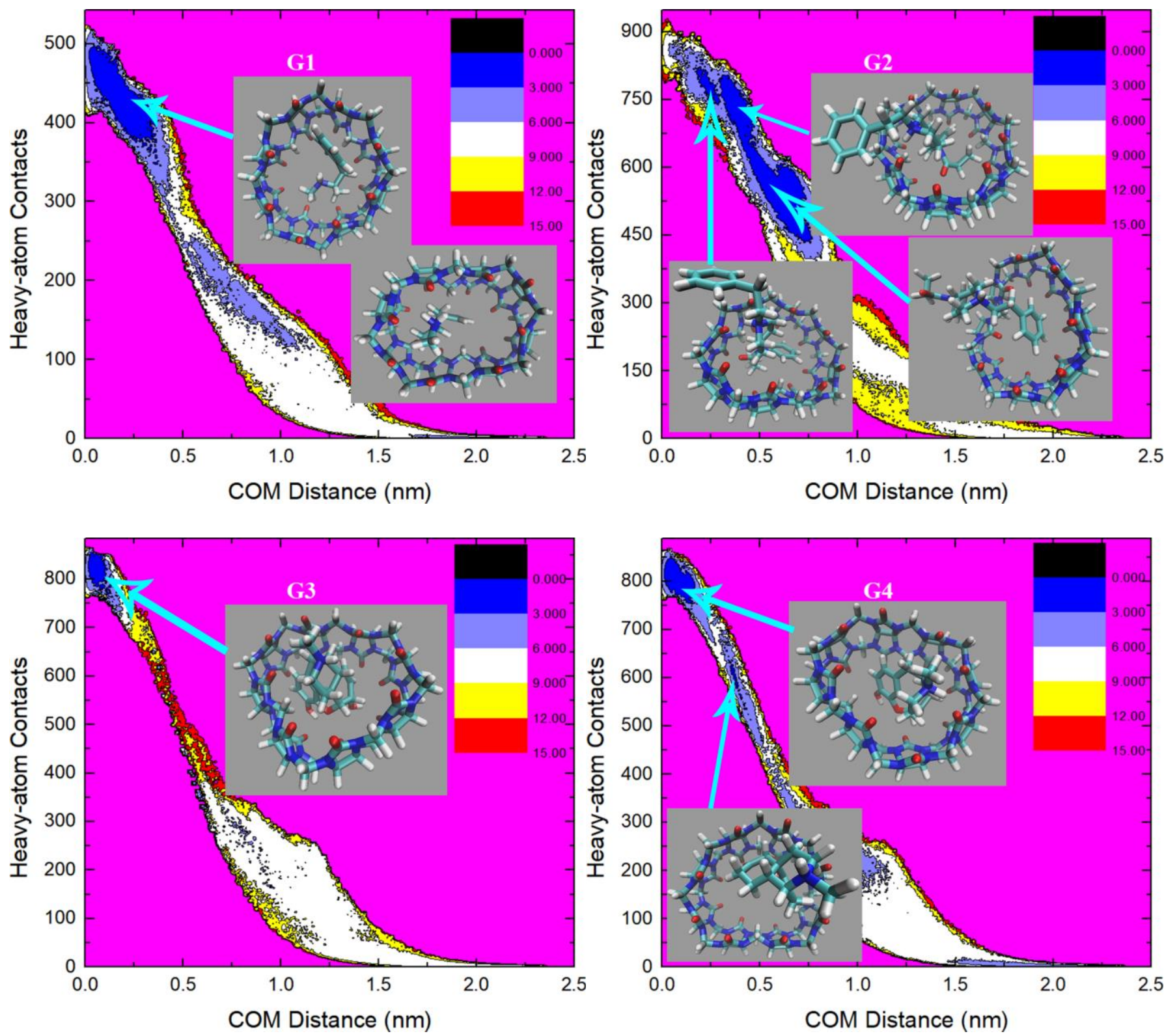

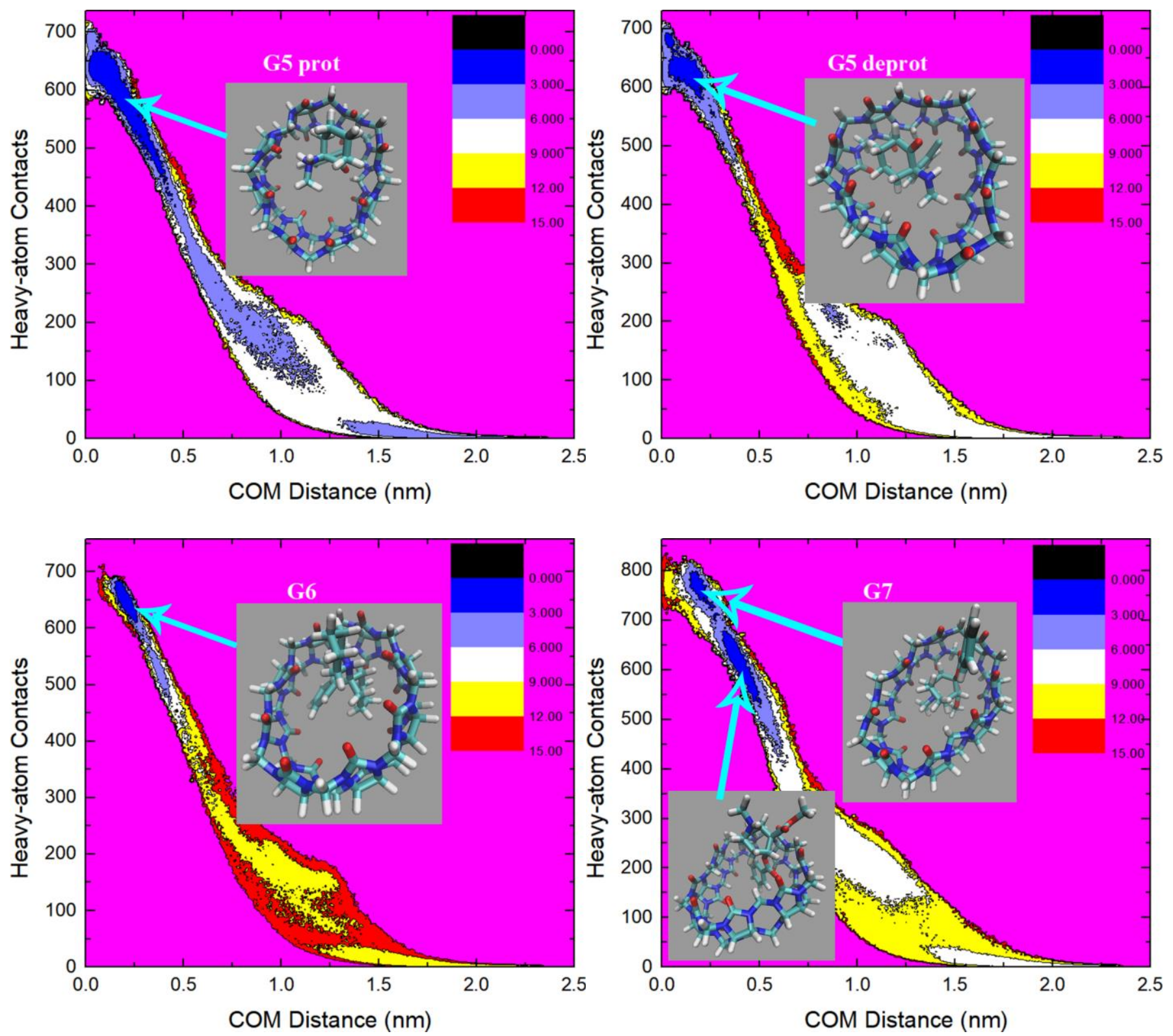
Fig. S11. 2D $\rho-C$ free energy surfaces in kcal/mol obtained with the FM-BLYP parameter set. Representative structures extracted from the global minimum and the other low-energy local minima are also shown. The binding poses obtained under the FM-BLYP parameter set are similar to the GAFF2 ones, which agrees with the observations in unbiased simulations.
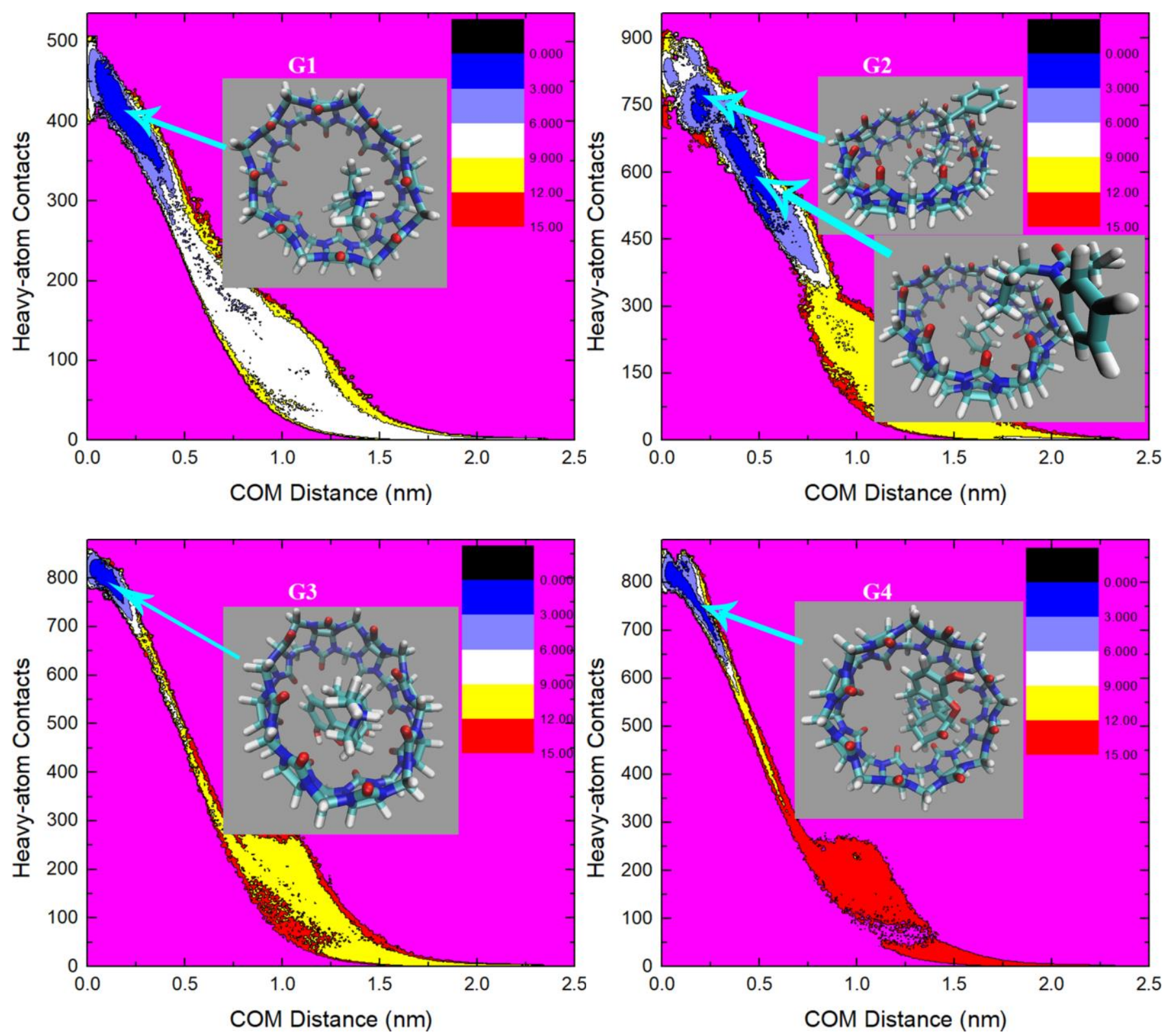

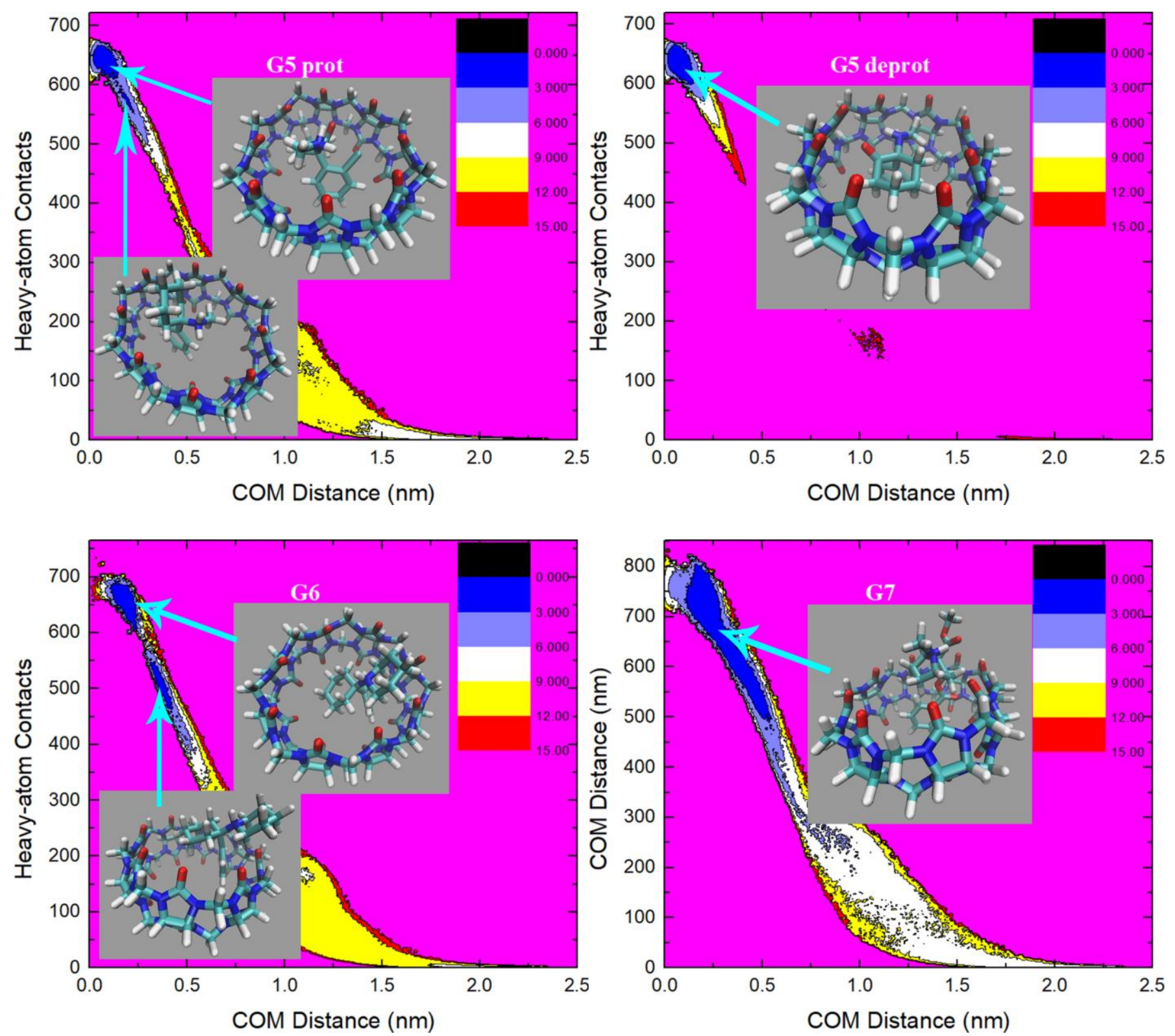